\title{
Multimorbidity, Immune Checkpoint Inhibitor Use, and Healthcare Expenditures among Older Patients with Late-stage Melanoma
}

\author{
Pragya Rai \\ West Virginia University, pr0001@mix.wvu.edu
}

Follow this and additional works at: https://researchrepository.wvu.edu/etd

Part of the Epidemiology Commons, Health Services Research Commons, Neoplasms Commons, Pharmacoeconomics and Pharmaceutical Economics Commons, and the Pharmacy Administration,

Policy and Regulation Commons

\section{Recommended Citation}

Rai, Pragya, "Multimorbidity, Immune Checkpoint Inhibitor Use, and Healthcare Expenditures among Older Patients with Late-stage Melanoma" (2020). Graduate Theses, Dissertations, and Problem Reports. 7789. https://researchrepository.wvu.edu/etd/7789

This Dissertation is protected by copyright and/or related rights. It has been brought to you by the The Research Repository @ WVU with permission from the rights-holder(s). You are free to use this Dissertation in any way that is permitted by the copyright and related rights legislation that applies to your use. For other uses you must obtain permission from the rights-holder(s) directly, unless additional rights are indicated by a Creative Commons license in the record and/ or on the work itself. This Dissertation has been accepted for inclusion in WVU Graduate Theses, Dissertations, and Problem Reports collection by an authorized administrator of The Research Repository @ WVU.

For more information, please contact researchrepository@mail.wvu.edu. 


\title{
Multimorbidity, Immune Checkpoint Inhibitor Use, and Healthcare Expenditures among Older Patients with Late-stage Melanoma
}

\author{
Pragya Rai \\ Dissertation submitted \\ to the School of Pharmacy \\ at West Virginia University \\ in partial fulfillment of the requirements for the degree of \\ Doctor of Philosophy in \\ Health Services and Outcomes Research \\ Usha Sambamoorthi, Ph.D, Chair \\ Kimberly M. Kelly, Ph.D \\ Virginia G. Scott, Ph.D, MS, BSPharm \\ Joanna Kolodney, MD \\ Chan Shen, Ph.D. \\ Department of Pharmaceutical Systems and Policy \\ Morgantown, West Virginia \\ 2020
}

Keywords: Melanoma, Metastatic melanoma, Multimorbidity, Multiple chronic conditions,

Immune checkpoint inhibitors, Healthcare expenditures, Older patients

Copyright 2020 Pragya Rai 


\section{ABSTRACT \\ Multimorbidity, Immune Checkpoint Inhibitor Use, and Healthcare Expenditures among Older Patients with Late-stage Melanoma}

\section{Pragya Rai}

With decades of unchanged cancer care with no added survival benefit, immune checkpoint inhibitors (ICI) changed the treatment landscape of late-stage melanoma in 2011. A key factor in determining the use of ICIs is the presence of pre-existing chronic conditions, which can influence the outcome. However, the prevalence of multimorbidity (defined as presence of two or more chronic conditions) among older patients with late-stage melanoma remains unknown. It also remains unknown if the presence of multimorbidity factors into the use of ICIs. Hospitalrelated factors associated with ICI use have been studied. Yet, patient-level factors, such as age, sex, marital status, which may play a more crucial role in the use of ICIs, remain unknown. Furthermore, ICI use may exacerbate healthcare expenditures for an already expensive condition, i.e., late-stage cancer. There are no studies exploring the effects of ICI on healthcare expenditures among the elderly. Therefore, this study had three main objectives (1) to examine the prevalence and type of pre-existing multimorbidity and the associated risk factors, (2) to assess the association of multimorbidity and other risk factors on ICI use, and (3) to assess the impact of ICI and multimorbidity on healthcare expenditures among older patients with latestage melanoma. Retrospective cohort studies were conducted using Surveillance, Epidemiology, and End Results cancer registry linked with fee-for-service Medicare claims for older (>66 years) patients with a 12-month pre-index and 12-month post-index period. Index date was the date of incident stage III/stage IV melanoma diagnosis between 2011 and 2015. Logistic regression was used to examine the associations of multimorbidity or ICI use to various patient-level factors. Generalized linear mixed models with gamma distribution and log-link was used to analyze adjusted relationships between ICI/multimorbidity and healthcare expenditures. In the first aim, an overwhelming majority (85\%) of older adults with late stage melanoma had pre-existing multimorbidity. The second aim concluded that only $6 \%$ of older adults with latestage melanoma received ICI. Multimorbidity was not significantly associated with ICI use. Factors positively associated with ICI use were lower age, social support in the form of spouse, residence in the Northeastern regions, and had dual eligibility compared to their counterparts. This study concluded that patient-level factors may play a significant role in decisions towards treatment of late-stage melanoma with ICI, regardless of multimorbidity. The third study reported that the average total, outpatient, home health, and inpatient healthcare expenditures in the pre-index period were significantly $(\mathrm{p}<0.001)$ lower than the expenditures in the post-index period. In addition, regardless of multimorbidity, the high expenditures in post-index period were influenced by the use of ICI. Therefore, ICI use was significantly associated with healthcare expenditures. In summary, multimorbidity is a growing concern for oncologists, especially among the elderly. The high prevalence of multimorbidity among elderly in our study points to the fact that multimorbidity should be factored into cancer care. Since multimorbidity is not included in cancer care guidelines, it does not play a role in receipt of ICI among older patients. However, the use of ICI significantly increases healthcare expenditures. To reduce costs, newer payment models that focus on value are being developed and tested. Future studies need to examine whether such models can achieve the triple aims of "better health, better value, and lower costs." 
The past few years have been nothing less than an exciting roller coaster ride, with some lows and many highs. I am surrounded by some of the most compassionate, intelligent, and dedicated people and I cannot imagine being where I am without them. This acknowledgement is my heartfelt "thank you" to each person mentioned (or not) here.

This dissertation would not have come into fruition without the support and oversight of five amazing committee members. First and foremost, I would like to thank Dr. Usha Sambamoorthi. I am forever grateful that she accepted to be my mentor at a time when I was struggling with uncertainty about my future. She has been instrumental in my growth as a researcher, especially during this dissertation phase. Her patience is unmatched, and she was always readily available to help me with any challenges that I faced while completing my dissertation. Working closely with her for the past few years, I am always amazed by how humble she remains and therefore inspires me to be a good leader like her in the future.

I would like to thank Dr. Kimberly M. Kelly for her constant support and oversight. She is the most empathetic person I know. She has always ensured that I feel safe and welcome, while working away from home. She is also an amazing cook and I will miss the breakfast meetings. But more importantly, it showed me her caring nature and I cannot express how it would sometimes lift my spirits. Despite being busy, she has always made time for my dissertation and provided valuable feedback, for which I am truly grateful. I would like to thank Dr. Virginia G. Scott for always being there for me. Whenever I had a personal problem, she would be my go-to person and she would listen patiently. She always greets you with the biggest smile and even if no words are spoken, her smile would let you know that everything will be okay. I am thankful for her encouragement and feedback on my dissertation. 
I would also like to thank Dr. Chan Shen and Dr. Joanna Kolodney for their guidance on this dissertation. Dr. Chan Shen was instrumental in obtaining the data for this dissertation. She was really kind with providing me access, day or night, weekdays or weekends, to my data. I have lost count of how many times I have bothered her with my requests, and she has always and patiently come through for me. Her immense help forms the backbone of this dissertation and I am forever grateful. I would also like to thank Dr. Joanna Kolodney for her oversight and feedback on this dissertation. She was always there to answer any questions I may have about melanoma or its treatment. She has been unbelievably supportive throughout this process and I would like to thank her for accommodating my requests in her busy schedule.

I am extremely thankful to West Virginia University, and all the faculty, staff, and students of the PSP department for providing me the necessary support to succeed. I am thankful to my former roommates and batchmates, Drishti Shah and Xiohui Zhao. They pushed me to better myself and inspired me to aim higher. I would also like to thank Yifan Zhang for always being there for me and teaching me rock climbing. I will always cherish my time with the three of them. I am thankful to each and every former and current student in the PSP department. Each one of you taught me something new and made my stay at WVU enjoyable.

My biggest thank you goes to my family, i.e., mom, dad, and sister. They are my biggest cheerleaders. Without their constant support, I would never have been able to reach where I am today. Words cannot express my gratitude towards them. Though there is incredible distance between us, they are always in my heart. Talking with them always fills me with hope and optimism. I am forever grateful for all the sacrifices they have made to help me achieve my dream. 


\section{Table of Contents}

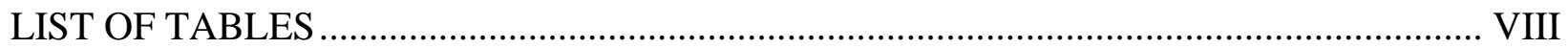

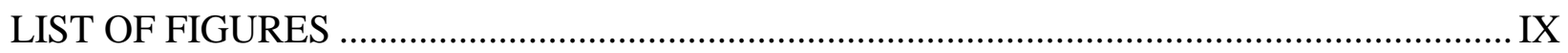

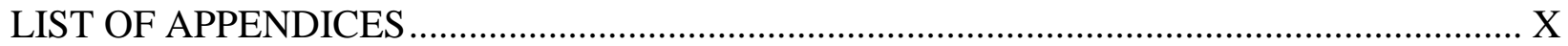

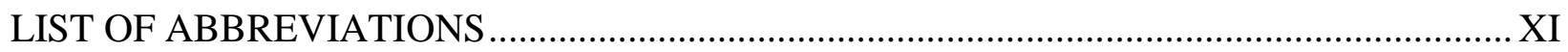

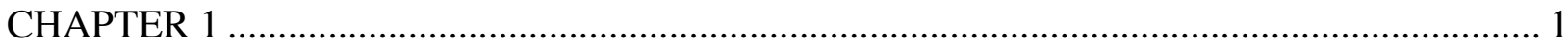

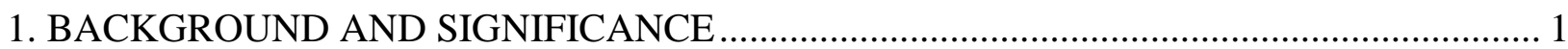

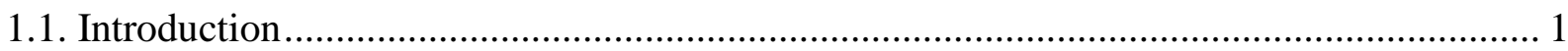

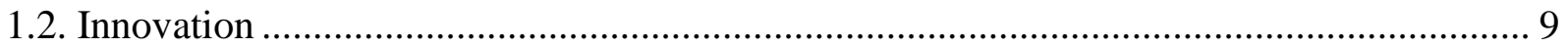

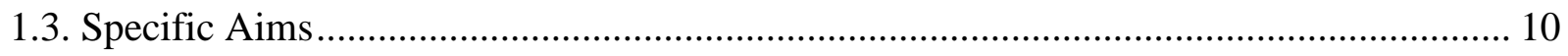

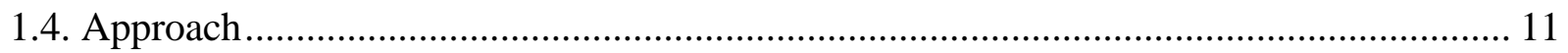

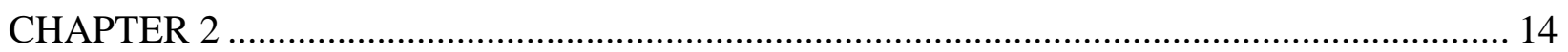

2. PREVALENCE AND RISK FACTORS FOR MULTIMORBIDITY IN ELDERLY US

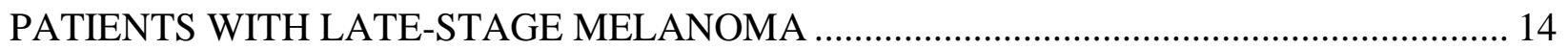

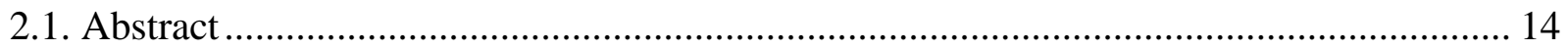

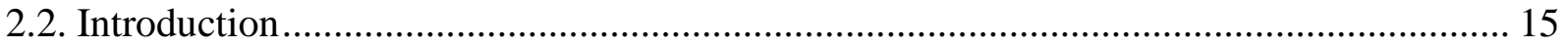

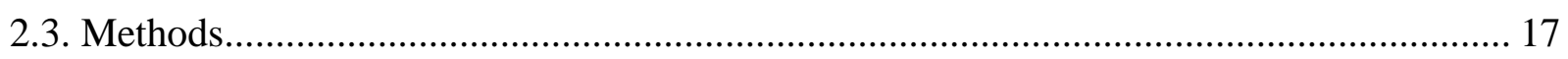

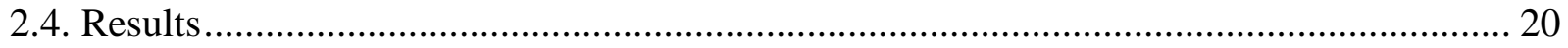

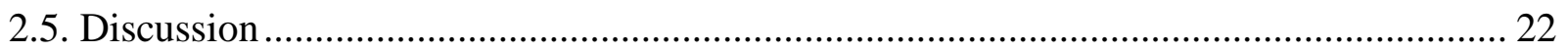

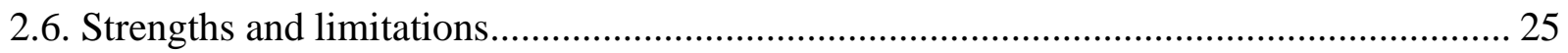

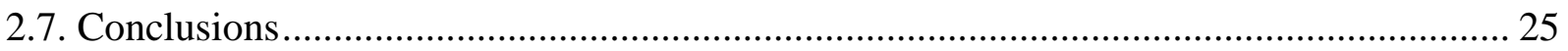

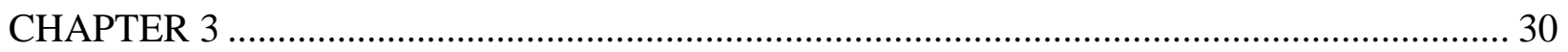




\section{FACTORS ASSOCIATED WITH IMMUNE CHECKPOINT INHIBITOR USE AMONG}

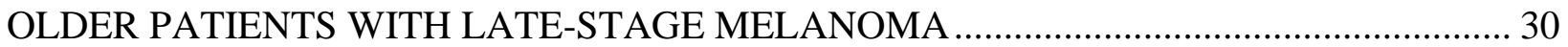

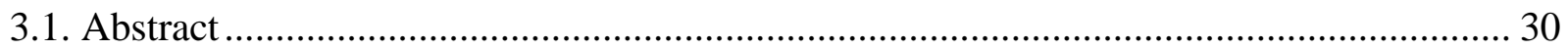

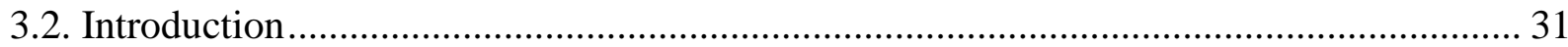

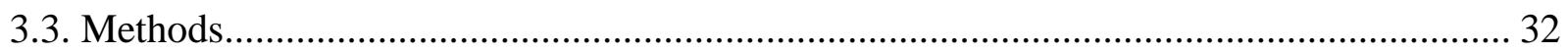

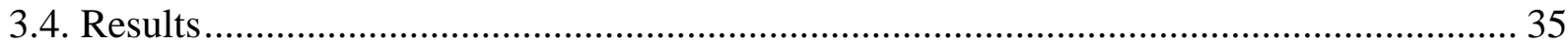

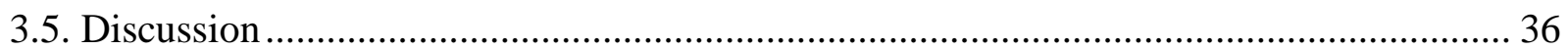

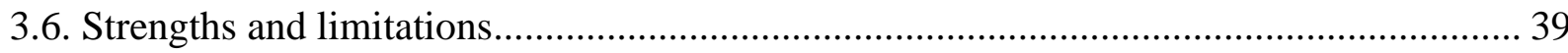

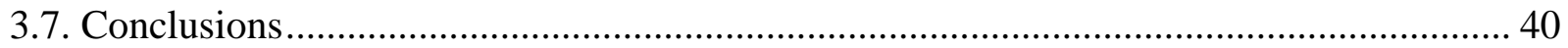

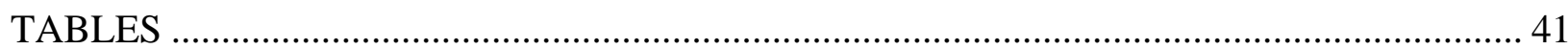

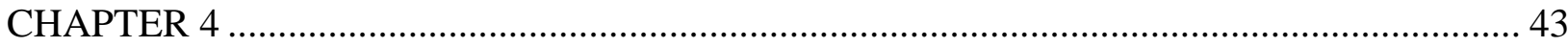

4. IMMUNE CHECKPOINT INHIBITOR USE, MULTIMORBIDITY, AND HEALTHCARE EXPENDITURES AMONG OLDER ADULTS WITH LATE-STAGE MELANOMA ............. 43

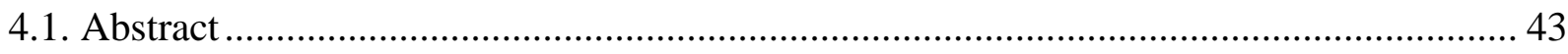

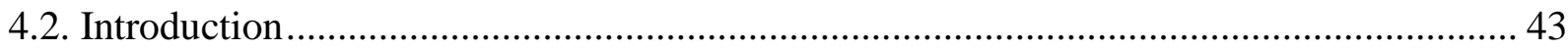

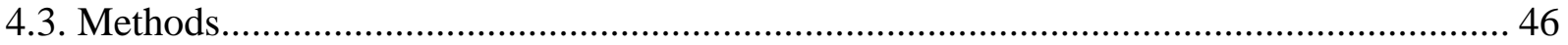

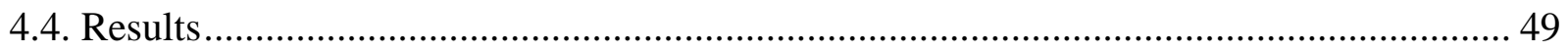

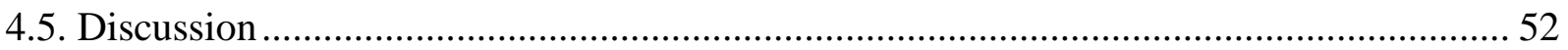

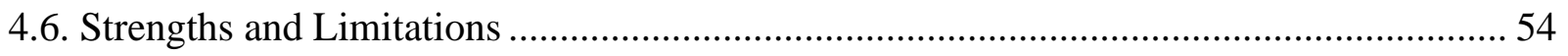

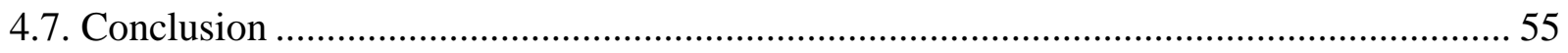

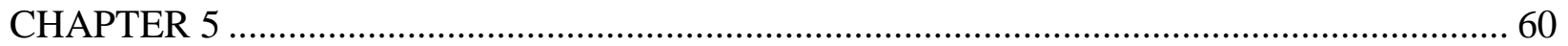

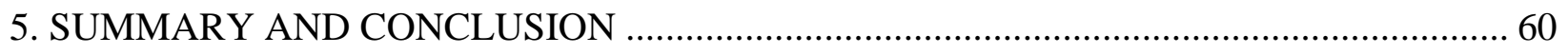

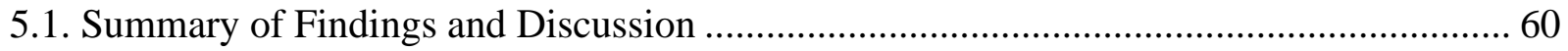




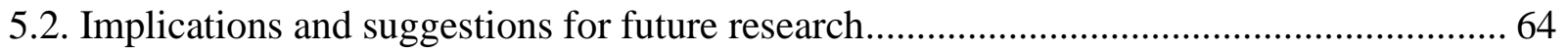

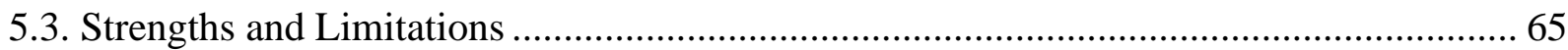

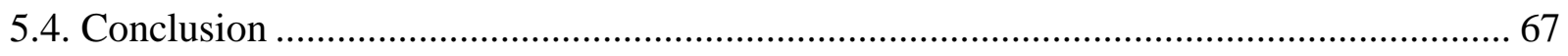

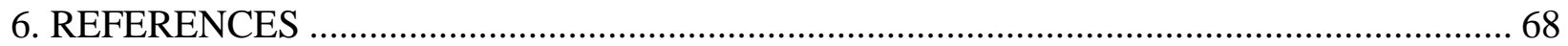

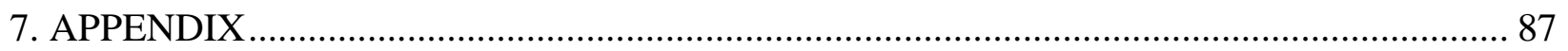




\section{LIST OF TABLES}

Table 2.1: Percent with Multimorbidity by Selected Patient-level Characteristics and Adjusted Odds Ratios (AOR) and 95\% Confidence Intervals (CI) from Logistic Regression on Any Multimorbidity Older Adults (age $\geq 66$ years) with Incident Latestage Melanoma during 2011 and 2015 using Linked Surveillance, Epidemiology, and End Results and Medicare Claims Database.

Table 2.2: Percent with Type of Multimorbidity by Selected Patient-level Characteristics Older Adults (age $\geq 65$ years) with Incident Late-stage Melanoma during 2011 and 2015 Linked Surveillance, Epidemiology, and End Results and Medicare Claims Database.

Table 2.3: Adjusted Odds Ratios (AOR) and 95\% Confidence Intervals (CI) from Separate Multivariable Logistic Regressions on Type of Multimorbidity Older Adults (age $\geq 65$ years) with Incident Late-stage Melanoma during 2011 and 2015 Linked Surveillance, Epidemiology, and End Results and Medicare Claims Database....

Table 3.1: Percent with Immune Checkpoint Inhibitor (ICI) use by Selected Patientlevel Characteristics among Older Adults (age >65 years) with Incident Late-stage Melanoma during 2012 and 2015 Linked Surveillance, Epidemiology, and End Results and Medicare Claims Database.

Table 3.2: Unadjusted Odds Ratio (OR), Adjusted Odds Ratios (AOR), and 95\% Confidence Intervals (CI) from Logistic Regressions on Immune Checkpoint Inhibitor use. Older Adults (age >65 years) with Incident Late-stage Melanoma during 2012 and 2015. Linked Surveillance, Epidemiology, and End Results and Medicare Claims Database.

Table 4.1: Parameter estimates of select variables of GLMM on average total healthcare expenditures. 


\section{LIST OF FIGURES}

Figure 1.1: Adapted determinants of health outcomes and chronic disease model and Andersen's healthcare utilization model.........................................

Figure 4.1: Schematic of the study design. Each individual was observed for 24 months with a 12-month pre-index and 12-month post-index period. Healthcare expenditures and selected independent variables were measured repeatedly every 120 days during the pre-index ( $\mathrm{t} 1, \mathrm{t} 2$, and $\mathrm{t} 3$ ) and post-index ( $\mathrm{t} 4, \mathrm{t} 5$, and $\mathrm{t} 6)$ periods, yielding a total of six repeated measures for every individual.

Figure 4.2: (a) Mean Total and Type of Healthcare Expenditures Over time; (b) Mean Total Healthcare Expenditures by ICI user status.

Figure 4.3: Mean total healthcare expenditures by ICI-Multimorbidity groups during

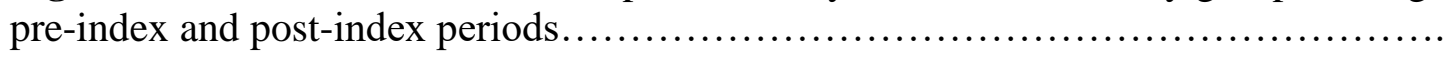




\section{LIST OF APPENDICES}

Appendix 7.1: List of autoimmune diseases

Appendix 7.2: Codes for identification of chemotherapy and radiation for the treatment of late-stage melanoma.

Appendix 7.3: Percent with Immune Checkpoint Inhibitor (ICI) use by Selected Patient-level Characteristics among Older Adults (age >65 years) with Incident Latestage Melanoma during 2012 and 2015. Linked Surveillance, Epidemiology, and End Results and Medicare Claims Database.

Appendix 7.4: Parameter estimates of select variables of GLMM on average outpatient expenditures

Appendix 7.5: Parameter estimates of select variables of GLMM on average home healthcare expenditures.

Appendix 7.6: Parameter estimates of select variables of GLMM on average inpatient expenditures 


\section{LIST OF ABBREVIATIONS}

ACS

AD

AHRF

AOR

CI

CMS

CVD

GLM

GLMM

HCPCS

ICD

ICI

irAE

$\mathrm{MHC}$

$\mathrm{NCCN}$

OCM

OR

$\mathrm{PHC}$

RCT

SEER

US
American Community Survey

Autoimmune diseases

Area Health Resource File

Adjusted odds ratio

Confidence interval

Centers for Medicaid and Medicare Services

Cardiovascular diseases

Generalized linear models

Generalized linear mixed models

Healthcare common procedure coding system

International Classification of Diseases

Immune checkpoint inhibitors

Immune-related adverse events

Mental health conditions

National Cancer Comprehensive Network

Oncology Care Model

Odds ratio

Physical health conditions

Randomized clinical trials

Surveillance, Epidemiology, and End Results

United States 


\section{CHAPTER 1}

\section{BACKGROUND AND SIGNIFICANCE}

\subsection{Introduction}

\section{Melanoma Prevalence and Incidence}

Melanoma is a type of skin cancer which arises from uncontrollable proliferation of melanocytes. ${ }^{1}$ Historically a rare cancer, its incidence has risen faster than any other cancer. ${ }^{1}$ This rise in incidence has been primarily attributed to tanning beds, though other risk factors such as sun exposure and other environmental factors cannot be ruled out. ${ }^{1,2}$ The incidence rose from 1 in 1,500 in 1935 to 1 in 30 in $2015 .{ }^{3}$ In 2020, approximately 100,000 Americans will be diagnosed with melanoma. ${ }^{4,5}$ This rise in incidence has made melanoma the fifth most common cancer in the United States (US). ${ }^{5}$ If caught early, prognosis of melanoma is favorable. ${ }^{3,6}$ However, once melanoma has metastasized, treatment becomes difficult with a grim long-term prognosis; median overall survival rates range from 6 to 8 months. ${ }^{7,8}$ Further, the 1- and 2-year survival rates for patients with metastatic melanoma were $23 \%$ and $9 \%$ respectively. ${ }^{9}$

\section{Melanoma in the elderly}

Globally, the greatest burden of melanoma falls on residents of Australia, Europe, New Zealand, the elderly and male population. ${ }^{10}$ The incidence in the older population continues to rise in the US while the incidence in younger population appears to be leveling off. ${ }^{11}$ Nearly $50 \%$ of all melanoma deaths in the US occur in white men older than 50 years. ${ }^{11}$ One of the reasons for higher death rates among the older population (>65 years) is immunosenescense, which means as we age, our immune system declines. ${ }^{11}$ Therefore, older patients have reduced capacity to fight infections and malignancy. Another reason for the high mortality rate among the elderly is that several poor prognostic factors, including non-superficial spreading histology, 
higher Clark invasion level, lower Breslow thickness, and presence of ulceration, were significantly observed in the elderly.

In addition, as patients age, the factors of primary melanoma become more advanced and melanoma is more likely to develop poorer histological features. ${ }^{12}$ Therefore, older patients are more likely to be diagnosed with late-stage (Stage III/IV) melanoma. ${ }^{12}$ A study noted that older patients had higher primary tumor stage, higher Breslow thickness, ulceration, and poorer survival compared to younger (18-64 years) patients. ${ }^{12}$ Therefore, this dissertation will focus on older patients with late-stage melanoma.

\section{Treatment of late-stage melanoma}

Once melanoma has been diagnosed, there are multiple options for treatment. Prior to 2011, management of metastatic melanoma included single-agents (alkylating agents, microtubule disrupting agents, platinum analogs, and nitrosoureas), as well as combination chemotherapy along with surgery and radiation therapy. ${ }^{13,14}$ However, these therapies did not significantly improve survival and were often associated with high toxicity. ${ }^{14-16}$ This led to the advent of immune checkpoint inhibitors (ICI; including ipilimumab, nivolumab, and pembrolizumab) since 2011, which have significantly improved survival with less toxic side effects. ${ }^{17,18}$ A departure from chemotherapy, ICIs work by energizing the immune system of the body to fight off the cancer cells. ${ }^{17,18}$ Recent data of 5.3 years median follow-up for ipilimumab, an anti-CTLA-4 agent, yielded significantly better distant metastasis free survival compared to standard chemotherapy, with no additional toxicities reported since the initial report at 2.3

years. ${ }^{19,20}$ Even superior overall survival, progression free survival, objective response rates, and better safety profile were observed with anti-PD-1/PD-L1 agents (nivolumab and pembrolizumab). ${ }^{20-22}$ This led many researchers to explore the efficacy of combination ICI. 
Early data from ongoing randomized clinical trials (RCTs) have shown durable response with combination ipilimumab and nivolumab. ${ }^{23}$ Such promising results from RCTs led to the integration of ICI (monotherapy or combination) as the first-line treatment for metastatic melanoma. ${ }^{24,25}$

\section{Multimorbidity}

People often live with two or more chronic conditions. Aging population and increase in life expectancy means that the number of people living with increasing number of chronic conditions will continue to rise. ${ }^{26}$ This "multimorbidity" or the coexistence of two or more chronic conditions in the same individual will have an impact on their safety in primary and specialty care. The safety concerns arise due to multiple reasons, including ${ }^{26}$

- polypharmacy, which may lead to poor medication adherence and adverse drug events,

- complex management regimens,

- more frequent and complex interactions with health care services leading to greater susceptibility to failures of care delivery and coordination,

- the need for clear communication and patient-centered care due to complex patient needs,

- demanding self-management regimens and competing priorities, and

- more vulnerability to safety issues due to poor health, advanced age, cognitive impairment, limited health literacy, and comorbid depression or anxiety.

Therefore, recent years has seen an increase in research on multimorbidity to alleviate the safety concerns for such individuals. 


\section{Multimorbidity in the elderly}

Studies have shown that prevalence of multimorbidity is on the rise worldwide. A study conducted in one developed country reported that almost $25 \%$ of the entire population has multimorbidities. ${ }^{26}$ Recent literature has shown high levels of multimorbidity in low- and middle-income countries. ${ }^{26}$ Similar trend has also been observed in high-income countries. For example, in Sweden, the prevalence of multimorbidity was $56.3 \%$ among adults aged 35 to 75

years. ${ }^{27}$ In the United States (US), one in four adults were reported to have multimorbidity, with $68 \%$ of the elderly individuals having two or more chronic conditions and $36 \%$ having four or more chronic conditions. ${ }^{28}$ Another study reported similar findings among Medicare beneficiaries: $62 \%$ aged 65 to 74 years, $75.7 \%$ aged 75 to 84 years, and $81.5 \%$ aged 85 years and above had multimorbidity. ${ }^{29}$ In addition, female Medicare beneficiaries had higher prevalence of multimorbidity compared to males. ${ }^{29}$

Such high prevalence has serious consequences on health outcomes of the elderly. Studies have shown that multimorbidity is associated with an increased risk of death, disability, poor functional status, poor quality of life, adverse drug events, and other adverse outcomes. ${ }^{29-31}$ Despite efforts to reduce multimorbidity among the elderly, the condition remains rampant. Therefore, studies as this are needed to provide information on the impact of multimorbidity on newly diagnosed conditions, such as cancer.

\section{Late-stage melanoma and multimorbidity}

\section{Prevalence of multimorbidity among older patients with late-stage melanoma}

The prognosis, treatment, and health outcomes (including survival) of patients with latestage melanoma depends on a number of factors, including age, tumor characteristics, and presence or absence of comorbidities. ${ }^{29,32}$ The presence of comorbidities influence cancer 
detection, treatment, and progression of cancer. This in turn can affect the prognosis and longterm survival of the patients. Patients with comorbidities are at higher risk of complications and lower performance status, may have decreased quality of life, and may face more life-threating conditions. Despite the importance of considering comorbidities in treatment and prognosis of melanoma, the prevalence of comorbidities has received little attention.

There are few studies on the impact of comorbidities of melanoma stage of diagnosis, treatment, and health outcomes. These studies reported that the prevalence of chronic conditions varies from $19 \%$ to $80 \%$ among adult patients with malignant melanoma. ${ }^{25,33-36}$ These studies did not focus on late-stage melanoma because of poor survival among those patients. However, the advent of ICIs may result in an increase in late-stage melanoma survivors. Therefore, identifying prevalent conditions in patients with late-stage melanoma will allow healthcare professionals to tailor their long-term care for each individual.

Moreover, the studies do not usually include mental health conditions. Literature also suggests that those with mental health conditions may have barriers to cancer care. ${ }^{37}$ However, most studies on patients with cancer have focused only on anxiety and depression; the presence of other mental health conditions, such as dementia, can also lead to less-aggressive cancer treatment because of compromised informed consent and adverse effects. ${ }^{38-41}$ Therefore, estimating the prevalence of any type of multimorbidity can be useful to the providers as well as patients.

In addition, one of the conditions worrisome for oncologists is autoimmune disease. This is because ICI therapy can induce autoimmune side effects affecting almost any organ system, specifically aberrant activation of immune cells against self-antigens, referred to as "immunerelated adverse events (irAE)". ${ }^{42}$ Patients with pre-existing autoimmune diseases may be at a 
greater risk for developing irAEs. ${ }^{43}$ Therefore, these patients were largely excluded from RCTs of ICI. However, a recent study reported that the prevalence of autoimmune diseases has been increasing from $17.1 \%$ in 2004 to $28.3 \%$ in $2014 .{ }^{36}$ Although autoimmune diseases are more frequent in the older patients, occurrence of autoimmune disease multimorbidity remains unknown. Therefore, a study which included a comprehensive list of mental health conditions and autoimmune diseases is necessary because studies often include only limited mental health conditions and no autoimmune diseases. In addition, examining factors (i.e., risk profile) other than age that may be associated with multimorbidity can help in assessing disease burden, surveillance, treatment decisions, and survivorship plans of patients with late-stage melanoma, justifying rationale for Aim 1.

\section{Factors associated with ICI use among older patients with late-stage melanoma}

Since the approval of ICI in 2011, literature on the improved survival associated with ICIs have increased exponentially. It stands to reason that the use of ICI in real-world setting may be commonplace. However, that is not the case; adoption of ICIs has been slow. We speculate few reasons for this slower diffusion of innovation. First, with their strict inclusion and exclusion criteria, RCTs of ICIs did not provide robust evidence on the use and impact of ICI among the elderly. Additionally, multimorbidity is prevalent among the older patients. Layering the management of melanoma onto the management of a patient with multimorbidity further compounds the complexity of care. The interplay between treatment approaches used for melanoma care and those used for multimorbidity can be complicated. In patients with preexisting multimorbidity, a new diagnosis of cancer adds a new dynamic, with the primary care physicians and other specialists shifting into supportive roles, to deliver non-cancer care in the 
context of cancer treatment. ${ }^{44,45}$ With lack of evidence from RCTs, decision-making on choosing ICI treatment for care may become difficult.

In addition, certain patient-level factors may be considered as a source of slower adoption of newer therapies. Studies on other cancers have shown some subgroups may be less likely to receive treatment. For example, age and racial disparities on treatment received was reported among older patients with late-stage pancreatic adenocarcinoma. ${ }^{46}$ Patients below the age of 80 years and White non-Hispanics were more likely to receive treatment than those above 80 years of age and other race/ethnicity. ${ }^{46}$ Similarly, a study among older patients with bladder cancer reported better survival among married patients than unmarried ones, because of greater likelihood of receiving treatment. ${ }^{47}$ Financial aspects potentially affecting the care setting for late-stage melanoma patients may also be a potential reason. ${ }^{48}$ Insured patients are more likely to receive ICIs compared to uninsured or underinsured patients. ${ }^{49}$ With evident disparities in receipt of treatment, it is critical to know whether some subgroups lag in the diffusion of innovative therapies like ICIs, so that oncologists and patients alike can make informed decisions when considering ICIs as the treatment option, thus justifying rationale for Aim 2.

\section{Healthcare expenditure associated with ICI among older patients with multimorbidity and late-stage melanoma}

The economic burden associated with cancer is substantial. There is robust evidence that cancer increases healthcare expenditures. The costs differ by type and stage of cancer. Therefore, although the cost of malignant melanoma is the lowest compared to other cancers, metastasis of melanoma significantly increases healthcare expenditures. ${ }^{50-52}$ In fact, $55 \%$ of the annual direct cost for treating melanoma arose from late-stage melanoma and one-third of the total cost was related to the end-of life melanoma treatment. ${ }^{53}$ The annual treatment costs ranged 
from $\$ 44.9$ million among Medicare patients with existing melanoma to $\$ 932.5$ million among newly diagnosed cases across all groups. ${ }^{54}$ Among Medicare beneficiaries, the average perpatient melanoma charges were $\$ 2,194$ during the initial four months of treatment and increased to $\$ 3,933$ during the terminal six months of treatment in $2010 .{ }^{55}$

Such costs are bound to increase with time due to inflation. However, recent years has seen a substantial increase in cancer-related healthcare costs. The high healthcare expenditures for late-stage melanoma may be driven by many factors such as high cost of ICIs, the associated administration costs for these drugs, and supporting care (monitoring and testing). ${ }^{56}$ One of the concerns are the high costs of ICIs. According to Dr. Leonard Saltz, the costs of newer therapies "approximately 4,000 times the cost of gold" ${ }^{57} \mathrm{He}$ calculated that the cost for a typical patient receiving the CheckMate067 combination (ipilimumab and nivolumab) would have been $\$ 295,566 .{ }^{57}$ With a $20 \%$ co-pay, the out-of-pocket cost to the patients would have totaled $\$ 60,000$. In addition, serum biomarker testing and enzyme level monitoring are common before and during treatment of late-stage melanoma. ${ }^{58}$ These functions also factor into the cost of melanoma care. Therefore, this amounted to $\$ 174$ billion for treating late-stage melanoma for one year only..$^{57}$

Further, the presence of multimorbidity may further exacerbate the costs. Healthcare resource utilization is high among patients with multimorbidity, with frequent visits to specialists and higher hospitalization rates. Therefore, there was a positive associated between multimorbidity and healthcare costs, with an average increase of $\$ 1,774$ in total costs for each body system affected by chronic conditions. ${ }^{59}$ It is reasonable that older adults with multimorbidity treated with ICIs will therefore incur the highest healthcare expenditures. 
It should be kept in mind that almost no patients pay for their treatments. Rather, the society collectively pay the price for the expensive therapies. When an individual gets a treatment, others pay through insurance premiums and/or taxes that support Medicare, Medicaid, or other government programs. Therefore, when medical costs increase, so do the insurance premiums. These rising premiums may in turn harm the very people who need it the most, by reducing their access to care because they may be unable to purchase coverage. Examining the healthcare expenditures and the reason behind the increase will allow various stakeholdershealthcare professionals, payers, organization- to identify and condemn practices with unreasonable costs, thus justifying the rationale for Aim 3.

\subsection{Innovation}

\section{a) Comprehensive evaluation of chronic conditions among older patients with late-}

stage melanoma: This is the first study to include a comprehensive list of physical and mental health conditions when evaluating the prevalence of multimorbidity. In addition, the updated list includes the autoimmune diseases based on the current challenges with ICI use.

b) Novel factors of ICI adoption: Studies have evaluated the role of healthcare practices in adopting ICIs. However, patient-level factors have not been evaluated. As shared decision-making is common practice, patient-level factors that influence the adoption of newer therapies might provide oncologists with leads to make headway on improving uptake of ICIs.

c) Fill a significant knowledge gap in literature: Given that no studies have examined multimorbidity among older patients with late-stage melanoma, the proposed study will fill an important knowledge gap in the literature by identifying the prevalence of various chronic conditions in such patients. 


\subsection{Specific Aims}

Aim 1.1: Estimate the prevalence and type of multimorbidity prior to the diagnosis of late-stage melanoma

Aim 1.2: Evaluate the association of patient-level factors to multimorbidity in older patients with late-stage melanoma

Hypothesis 1.2: In the fully adjusted model which will include all the independent variables, age will be significantly associated with multimorbidity among older patients with late-stage melanoma.

Aim 2: Examine the association of multimorbidity and other factors to ICI use that cover years from the initial introduction in 2011 to 2015 among older patients with latestage melanoma

Hypothesis 2: In the fully adjusted model, year of diagnosis, age, marital status, dual eligibility, and region of residence will be significantly associated with ICI use among older patients with late-stage melanoma.

Aim 3.1: Estimate the impact of ICI use on healthcare expenditures during pre- and post-late-stage melanoma diagnosis periods among older patients with late-stage melanoma Hypothesis 3.1: ICI use will be associated with high expenditures throughout the treatment period compared to those without ICI use.

Aim 3.2: Estimate the impact of ICI-multimorbidity interaction on healthcare expenditures during pre- and post-late-stage melanoma diagnosis periods among older patients with late-stage melanoma 
Hypothesis 3.2: Patients who used ICI and had multimorbidity will have higher expenditures throughout the treatment period compared to ICI non-users and those without multimorbidity.

\subsection{Approach}

\section{Conceptual Frameworks Used to Guide the Selection of Variables}

A conceptual framework was created by adapting determinants of health outcomes and chronic disease model and Andersen's healthcare utilization model. ${ }^{60,61}$ According to these

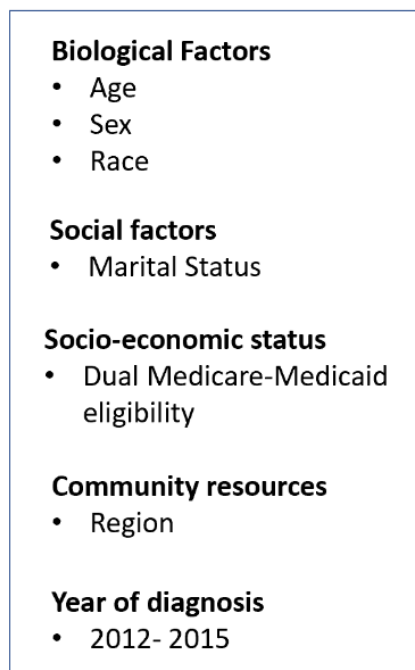

Healthcare utilization

- PCP visits

- Oncologist visits models, outcomes

such as presence of

multimorbidity,

receipt of novel

therapies (ICI), and

consequently

healthcare

Figure 1.1: Adapted determinants of health outcomes and chronic disease model and Andersen's healthcare utilization model expenditures are based

on the presence or

absence of multiple

factors. These factors included biological factors, social factors, community resources, socioeconomic status, and year of diagnosis. Biological factors consisted of age (66-69, 70-74, 75-79, and $\geq 80$ years), sex (male/female), and race (white/non-White). Social factors included marital status (married/not married). Community resources included regions (Northeast, South, West, and Northcentral). Dual Medicare/Medicaid enrollment (yes/no) was a proxy for low economic status. Years of incident melanoma diagnosis (2012-2015) was used to control for changes in 
practice patterns. Aim 3 also included healthcare utilization (visits to primary care physician and oncologists).

\section{Data sources}

Data from multiple sources were used to accomplish the study aims.

Surveillance, Epidemiology, and End Results (SEER): SEER is a cancer registry and provides data on clinical variables related to cancer. SEER data consists of various files, which contain information on patients and cancers. Patient Entitlement and Diagnosis Summary File provides information for each person's date of birth, sex, race, and state of residence. This file also includes information on Medicare eligibility, reason for Medicare entitlement, and health maintenance organization (HMO) enrollment by month. In addition, these files also contain geographically-based (ZIP code and census tract level) socioeconomic information. The file contains exhaustive information on cancer, such as diagnosis date, cancer stage, tumor size, number of positive lymph nodes, and tumor histology.

SEER-Medicare Claims: Medicare Enrollment File: Medicare is the federally funded program that provides health insurance to the elderly, persons with end-stage renal disease, and some disabled. For persons aged 65 and over, nearly 90\% are Medicare eligible. Medicare claims provides information on Part A coverage (hospital, skilled nursing facility, hospice and some home healthcare), Part B coverage (physician and outpatient services), Part C (HMO enrollment, most of whom have fee-for-service coverage), and Part D (prescription drug) coverage. Medicare claims database is the best source of information to estimate Medicare payments among the elderly for cancer and other disease states. To link SEER with Medicare data, identifiers contained in the Medicare's master enrollment file is matched with individual identifiers for all persons in their files. 
American Community Survey (ACS) Census Tract Files: The ACS census tract and census zip code files were linked to PEDSF files by geographic codes, such as, state and county. These files provided information on the census tract median household income and education level.

Area Health Resource File (AHRF): The AHRF is a publicly available data file provided by Department of Health and Human Services and contains county, state and national files. The AHRF provides more than 6,000 variables for each of the nation's counties. The AHRF contains information such as health facilities, health professions, and socioeconomic and environmental characteristics. The basic file contains geographic codes and descriptors that may be used to link it to other files and to aggregate counties into various geographic groupings. This study used the AHRF variables to measure health care infrastructure that can influence the detection of multimorbidity, ICI use and expenditures. 


\section{CHAPTER 2}

\section{PREVALENCE AND RISK FACTORS FOR MULTIMORBIDITY IN ELDERLY US PATIENTS WITH LATE-STAGE MELANOMA}

\subsection{Abstract}

Introduction: Presence of multimorbidity can affect prognosis, treatment, and outcomes of individuals with cancer. However, the prevalence and factors associated with multimorbidity among older late-stage melanoma is not well studied. We estimated the prevalence of any type of pre-existing multimorbidity (autoimmune disorder (AD), physical health conditions (PHC), and mental health conditions (MHC)) among older adults with late-stage melanoma in the United States. We further examined the association of patient-level factors to multimorbidity in latestage melanoma.

Methods: We derived data on older fee-for-service Medicare beneficiaries (age $\geq 66$ years) diagnosed with late-stage melanoma between 2011 and $2015(\mathrm{~N}=4,519)$ from the linked Surveillance, Epidemiology, and End Results cancer registry and Medicare claims. We defined multimorbidity as the prevalence of two or more chronic conditions prior to the diagnosis of melanoma. We used unadjusted and adjusted logistic regressions to examine the association of patient-level factors to multimorbidity.

Results: An overwhelming majority (85\%) of older patients with late-stage melanoma had multimorbidity. Pre-existing PHC multimorbidity (84\%) was the most prevalent, followed by $\mathrm{AD}(12 \%)$, and $\mathrm{MHC}(6 \%)$. Age and region were associated with any and PHC multimorbidity. Sex, marital status, and region were factors associated with pre-existing AD while sex, marital status, and dual eligibility were associated with MHC multimorbidity. 
Conclusions: Pre-existing multimorbidity was highly prevalent among older individuals with late-stage melanoma; prevalence rates and factors associated with multimorbidity varied by type of chronic conditions. This highlights the need for developing systematic approaches to optimizing care of older patients with late-stage melanoma and multimorbidity.

\subsection{Introduction}

Melanoma is a form of skin cancer with an alarmingly-rising incidence in the United States (US), making it the fifth most common cancer, with the highest occurrence among the elderly ( $\geq 65$ years). ${ }^{2,62,63}$ Although melanoma accounts for $2 \%$ of all skin cancers, late-stage melanoma is the most lethal, accounting for $90 \%$ of deaths. ${ }^{2}$ A key factor for treatment of patients with melanoma is having a pre-existing chronic condition. Pre-existing chronic conditions are highly prevalent in all cancers and have been shown to affect cancer stage at diagnosis, treatment, and outcomes; melanoma being no exception. ${ }^{32,64}$ The prevalence of chronic conditions in patients with melanoma varies from $19 \%$ to $80 \%{ }^{33,34}$ The presence of additional chronic conditions prevents physicians from aggressively treating melanoma, thereby increasing the risk of mortality. ${ }^{33,34}$

As patients age, the number of chronic conditions increases. ${ }^{29}$ Some studies focused on the presence of multimorbidity, defined as the presence of two or more chronic conditions, among patients with breast, bladder, and colorectal cancers. ${ }^{65-68}$ These studies also documented high prevalence of multimorbidity and associated adverse outcomes. However, to date no study has exclusively examined multimorbidity in patients with late-stage melanoma perhaps due to the poor survival of these patients. Studies of multimorbidity among late-stage melanoma patients are important and needed for reasons stated below. 
Immune checkpoint inhibitors (ICI) have changed the treatment landscape of late-stage melanoma, with significant improvements in survival. ${ }^{6,69}$ However, pre-existing multimorbidity may compete with cancer care because cancer treatment needs to focus not only on mortality but also treatment complications and quality of life. ${ }^{70}$ For example, ICIs affect the immune system. ${ }^{18}$ Patients with pre-existing autoimmune diseases (AD) have weaker immune systems, and this may deter their treatment with ICI, due to fears of higher mortality and worse quality of life post-treatment. ${ }^{42,71}$ As ICIs are often used in cancer patients with pre-existing AD seeking care in the real-world settings, ${ }^{42,43,72}$ it is critical to assess the prevalence of pre-existing $\mathrm{AD}$ in the elderly. Furthermore, literature also suggests that those with mental health conditions (MHCs) may have barriers to cancer care. ${ }^{37}$ However, most studies on patients with cancer have focused only on anxiety and depression; the presence of other MHCs, such as dementia, can also lead to less-aggressive cancer treatment because of compromised informed consent and adverse effects. $^{38-41}$ Therefore, estimating the prevalence of any type of multimorbidity can be useful to the providers as well as patients. Including a comprehensive list of MHCs and ADs is necessary because studies often include only limited MHCs and no ADs. In addition, examining factors (i.e. risk profile) other than age that may be associated with multimorbidity can help in assessing disease burden, surveillance, treatment decisions, and survivorship plans of patients with latestage melanoma.

Therefore, the objectives of the current study are to estimate the prevalence and type of multimorbidity prior to the diagnosis of late-stage melanoma and assess the association of patient-level factors to multimorbidity in older patients with late-stage melanoma. 


\subsection{Methods}

\section{Study Design}

We adopted a retrospective cohort design. The cohort consisted of older adults with incident late-stage (stage III/IV) melanoma diagnosis based on the American Joint Committee on Cancer $7^{\text {th }}$ Edition. Index date was defined as the date of incident late-stage melanoma diagnosis. Pre-index period was defined as 12 months before incident melanoma diagnosis. Multimorbidity, type of multimorbidity, and all independent variables were assessed during the pre-index period. The study was exempted by West Virginia University Institutional Review Board.

\section{Data Source}

Surveillance, Epidemiology, and End Results (SEER) cancer registry and Medicare claims files were used. The SEER data provided information on clinical variables related to cancer (example: stage of cancer at diagnosis); Medicare claims provided information on healthcare encounters of the beneficiaries when enrolled and using Medicare covered health services.

\section{Study Population}

We identified 41,848 individuals with incident melanoma diagnosis between 2011 and 2015 using ICD-O-3 site (C44.0 - C44.9) and histology (8720 - 8790) codes. Following the exclusion of those with local or regional (stage I/II) melanoma, non-incident melanoma, below the age of 66 years, not continuously enrolled in Medicare part A and Part B during the pre-index period, and diagnosed with late-stage cancer during autopsy, the final analytical cohort comprised 4,519 individuals with late-stage melanoma. 


\section{Measures}

\section{Dependent variables: Any, PHC, $M H C$, and AD multimorbidity}

Multimorbidity was defined as the presence of $\geq 2$ chronic conditions prior to the diagnosis of incident late-stage melanoma. We used the list of chronic conditions developed by the Multiple Chronic Conditions working group within the US Department of Health and Human Services Office of Assistant Secretary of Health. ${ }^{73}$ The list was developed based on the conditions that met the definition of chronicity, are prevalent, and are potentially amenable to public health. Based on the current challenges with ICIs, pre-existing AD was added, which were identified from a list of 131 conditions provided by the American Autoimmune-Related Disease Association (Appendix 7.1). ${ }^{74}$ All these conditions were identified with International Classification of Diseases (ICD), $9^{\text {th }}$ Edition.

Physical Health Conditions (PHC) consisted of pre-existing AD, arthritis, asthma, coronary artery disease, cardiac arrhythmias, diabetes, congestive heart failure, chronic kidney disease, chronic obstructive pulmonary disease (COPD), hepatitis, hyperlipidemia, hypertension, human immunodeficiency virus, obesity, osteoporosis, and stroke.

To explore the PHC most prevalent among those with multimorbidity, we created a taxonomy of PHC. The groups were: cardiovascular diseases (CVD - coronary artery disease, cardiac arrhythmias, congestive heart failure, and hypertension), endocrine diseases (diabetes, hyperlipidemia, and obesity), musculoskeletal diseases (arthritis, osteoporosis, and pre-existing $\mathrm{AD})$, and respiratory diseases (asthma and COPD).

Mental health conditions (MHC) consisted of anxiety, bipolar disorder, depression, psychoses, schizophrenia, and substance abuse. 
We also added PHC groups (CVD, endocrine, musculoskeletal, and respiratory diseases) and MHCs to explore any multimorbidity using combinations of dyads (e.g. CVD /endocrine diseases, MHC/respiratory diseases) and triads (e.g. CVD/endocrine diseases/MHC).

Indices of pre-existing conditions for each of the categories (PHC, MHC, and AD) were also created. These were derived by counting the number of conditions in each of the groups; PHC index ranged from 0-13, MHC index from 0-5, and AD index from 0-6.

\section{Independent variables}

These were identified at the pre-index period and included biological and social factors, community resources, socio-economic status, and year of diagnosis. Biological factors consisted of age (66-69, 70-74, 75-79, and $\geq 80$ years), sex (male/female), and race (white/non-white). Social factors included marital status (married/not married). Community resources included regions (Northeast, South, West, and Northcentral). Dual Medicare/Medicaid enrollment (yes/no) was used as a proxy for low economic status. Years of incident melanoma diagnosis (2012-2015) was used to control for changes in practice patterns.

\section{Statistics}

Chi-square tests were used to identify significant unadjusted associations of individual characteristics to multimorbidity. Multivariable logistic regressions were performed to determine the factors associated with multimorbidity. Parameter estimates are presented as adjusted odds ratios (AORs) after adjusting for all independent variables, with their corresponding 95\% confidence intervals $(\mathrm{CI}) ; \mathrm{p} \leq 0.05$ was considered statistically significant. All analyses were conducted using SAS 9.4 (SAS Institute Inc.). 


\subsection{Results}

The study population was predominantly male (64.2\%), White $(96.1 \%)$ and $\geq 70$ years (70.4\%). The mean number of chronic conditions (including PHCs, MHCs and ADs) was 4.26 with median of 4.0. The mean number of PHCs was 3.98 with median of 4.0. The prevalence of any $\mathrm{PHC}$ was $92.3 \%$; MHC was $20.8 \%$ and AD was $28.7 \%$. The most prevalent pre-existing condition was CVD (78\%) followed by endocrine diseases (72\%).

\section{Multimorbidity patterns}

Multimorbidity was highly prevalent $(85 \%)$ in the US elderly with late-stage melanoma. The prevalence of multimorbidity varied by type of chronic conditions: $84 \%$ had PHC multimorbidity, $12 \%$ had AD multimorbidity, and 6\% had MHC multimorbidity.

Among those with any multimorbidity, the most prevalent dyad combination was endocrine and musculoskeletal diseases combination (21.7\%) followed by CVD and endocrine disease combination (20.3\%). Among triads, CVD, endocrine, and musculoskeletal disease combination (22.6\%) was the most prevalent followed by endocrine, MHC, and musculoskeletal disease combination $(7 \%)$.

\section{Factors associated with any multimorbidity}

In unadjusted analyses, characteristics of individuals with and without multimorbidity differed by age and region (Table 2.1). A significantly higher proportion of Medicare beneficiaries aged $\geq 80$ years had multimorbidity compared to those in the age group 65-69 years $(89.4 \%$ versus $77.1 \%, \mathrm{p}<.0001)$. Multivariable logistic regression on any multimorbidity showed similar results (Table 2.1). Older adults in the age group 70-74 years (AOR, 1.41; 95\%CI, 1.12, 1.76), 75-79 years (AOR, 1.84; 95\%CI, 1.44, 2.35) and $\geq 80$ years (AOR, 2.55; $95 \% \mathrm{CI}, 2.04,3.18)$ were more likely to have pre-existing multimorbidity compared to those in 
the age group 65-69 years. Older adults residing in the Northcentral (AOR, 0.69; 95\%CI, 0.49, 0.96) and Western (AOR, 0.65; 95\%CI, 0.51, 0.83) SEER regions were less likely to have any multimorbidity compared to residents in the Northeastern parts.

\section{Factors associated with PHC multimorbidity}

In unadjusted analyses, characteristics of individuals with and without PHC multimorbidity differed by age and region (Table 2.2). Individuals with PHC multimorbidity had significantly higher proportion of those aged $\geq 70$ years and residing in Northeastern regions compared to those without PHC multimorbidity. Multivariable logistic regression on PHC multimorbidity showed similar results (Table 2.3). Older adults in the age group 70-74 years (AOR, 1.42; 95\%CI, 1.13, 1.77), 75-79 years (AOR, 1.81; 95\%CI, 1.42, 2.30) and $\geq 80$ years (AOR, 2.47; 95\%CI, 1.99, 3.06) were more likely to have pre-existing multimorbidity compared to those in the age group 65-69 years. Older adults residing in the Northcentral (AOR, 0.65; 95\%CI, 0.47, 0.90) and Western (AOR, 0.67; 95\%CI, 0.53, 0.85) SEER regions of the US were less likely to have PHC multimorbidity compared to residents in the Northeastern region.

\section{Factors associated with AD multimorbidity}

In unadjusted analyses, characteristics of individuals with and without AD multimorbidity differed by sex and region (Table 2.2). A significantly higher proportion of females and residing in Northcentral SEER regions compared had AD multimorbidity compared to men and those residing in Northeastern regions. Multivariable logistic regression on AD multimorbidity (Table 2.3) confirmed unadjusted associations; females (AOR, 1.81; 95\%CI, $1.48,2.22)$ were nearly two times as likely to have AD multimorbidity compared to older males. Unmarried older adults (AOR, 0.79; 95\%CI, 0.65, 0.97) and those residing in southern SEER 
regions (AOR, 0.58; 95\% CI, 0.42, 0.80) were less likely to have AD multimorbidity compared to married elderly and residents in the Northeastern regions.

\section{Factors associated with MHC multimorbidity}

In unadjusted analyses, characteristics of individuals with and without MHC multimorbidity differed by sex, marital status, and dual eligibility (Table 2.2). Elderly with MHC multimorbidity had significantly higher proportion of females, unmarried, and lower income status. Multivariable logistic regression on MHC multimorbidity showed similar results (Table 2.3). Older females (AOR, 1.88; 95\%CI, 1.46, 2.44) and those not married (AOR, 1.85; 95\%CI, 1.41, 2.44) were more likely to have MHC multimorbidity compared to older males and those married. Older adults with lower income status (AOR, 3.33; 95\%CI, 2.14, 5.19) were three times more likely to have MHC multimorbidity compared to those with higher income status.

\subsection{Discussion}

There is sparse information on prevalence of multimorbidity among individuals with melanoma, with no studies focusing on the elderly with late-stage melanoma. This is the first study to explore the prevalence and factors associated with the presence and type of multimorbidity among older adults with late-stage melanoma in the US. We found that 4 in 5 older adults with late-stage melanoma had pre-existing multimorbidity. Our estimates are higher than those found in non-US studies. For example, a Danish study reported multimorbidity in $9 \%$ of patients with melanoma. ${ }^{33}$ This study included adults (>18 years) and all stages of melanoma. In contrast, a German study reported multimorbidity in $57 \%$ of patients with melanoma. The difference in estimated prevalence rates among these studies may be due to the differences in the study population (all stages as opposed to late-stage and older adults as opposed to adults 18 years or older) and time-period (pre-existing versus co-existing conditions). 
The most common comorbid conditions in our study were CVD, endocrine, and musculoskeletal diseases or a combination of the three in dyads and triads. This is not surprising given that CVD, endocrine, and musculoskeletal diseases are highly prevalent in older adults. ${ }^{75,76}$ These diseases and cancer share modifiable risk factors, with some studies stating a bidirectional relationship between them. ${ }^{77-79}$ As heart disease, diabetes, and malignant cancers are among the top five leading causes of age-adjusted mortality, ${ }^{80}$ healthcare providers managing patients with late-stage melanoma may need to routinely monitor for the presence of these conditions and coordinate their care with the primary care physicians.

Among risk factors for any multimorbidity, age is well-established, with studies reporting that the number of chronic conditions increases with age, ${ }^{29,33}$ a trend noted in our study as well. Increasing number of chronic conditions led to late-stage cancer detection, as both the patient and the physician were distracted by the pre-existing conditions. ${ }^{81}$ Adding increasing age to the mix resulted in less aggressive treatment and a dismal chance of entering a clinical trial, as physicians had to grapple with physical frailty and polypharmacy as well in these patients. ${ }^{33,81}$ Therefore, oncologists should optimize the treatment of aging patients with multimorbidity to improve their outcomes.

Regions, namely Northcentral and Western SEER regions, were associated with lower odds of having multimorbidity. There are no exhaustive studies on SEER geographical disparities in late-stage melanoma cancer patients, and further research is needed to explain these results.

Our study also examined type of multimorbidity in terms of PHC, AD, and MHC. Risk factors for PHC multimorbidity were similar to any multimorbidity, perhaps because PHC was more prevalent than MHC, making up the bulk of multimorbidity index. Among the PHC, we 
further explored the prevalence of pre-existing AD among older patients. In 2014, the prevalence of pre-existing AD among individuals with metastatic melanoma was $20 \%$. $^{36,82}$ Our study echoed those findings among the elderly as well. Similar to a previous study on patients with metastatic melanoma and $\mathrm{AD}$, our study also notes sex and region as a risk factor for $\mathrm{AD}$ multimorbidity. ${ }^{36}$ In addition, this study also notes the presence of social support in the form of spouses as a risk factor for AD multimorbidity. Spouses are an important source of social support for patients with any chronic condition. ${ }^{83}$ Therefore, patients with multiple AD are more dependent on that social support to manage their conditions. ${ }^{83}$ Oncologists should consider these risk factors when deciding if a drug acting via the immune system is the best course of treatment for older patients.

Consistent with other studies, we observed $30 \%$ of older adults with late-stage melanoma were diagnosed with any MHC. ${ }^{84,85}$ Similar levels of MHC were also found in individuals with late-stage breast, ${ }^{38}$ colorectal, ${ }^{39}$ and prostate cancer. ${ }^{40}$ Sex as a risk factor was also observed with MHC multimorbidity, perhaps because MHC are more prevalent in females compared to males. ${ }^{86,87}$ In addition, lack of social support was a risk factor for MHC multimorbidity. Social deprivation is a known risk factor for MHC among patients with cancer. ${ }^{88,89}$ We also observed that dual eligibility was a risk factor for having MHC multimorbidity. Individuals with melanoma having Medicaid had worse prognosis compared to uninsured and non-Medicaid individuals, pointing to the importance of socioeconomic status in melanoma care. ${ }^{90}$ These risk factors should be assessed in order to reduce further psychological distress and devise appropriate follow-up interventions. 


\subsection{Strengths and limitations}

The present study has several limitations. First, SEER does not contain information on individual behavior such as smoking status, alcohol consumption, or functional status, all of which can further affect the risk of having multimorbidity. Second, we did not have access to individual-level socioeconomic factors, such as education and income. Third, we could not determine the severity of the conditions. Despite these limitations, this study has several strengths. Although few studies on comorbid conditions in individuals with melanoma exist, they did not focus on late-stage melanoma, perhaps because the prognosis was poor with low chances of survival. ${ }^{91}$ Therefore, prognostic burden of multimorbidity in such individuals was deemed less important. ${ }^{91}$ However, newer therapies such as ICIs will improve survivorship of patients with late-stage melanoma. Therefore, information on the prevalence and type of multimorbidity in such individuals can help both physicians and patients in choice of cancer treatment, survivorship plans, and quality of life care. We included an exhaustive list of PHC and MHC, including pre-existing AD.

\subsection{Conclusions}

Nearly 4 in 5 older adults with late-stage melanoma had any multimorbidity. The prevalence of multimorbidity varied by type of conditions with lowest in MHC and highest in PHC. One in 10 older adults with late-stage melanoma had AD multimorbidity, suggesting challenges to the management and decisions for treatment. Cardiovascular, endocrine, and musculoskeletal diseases were the most commonly occurring comorbid conditions, which share risk factors with cancer. While the risk factors for any multimorbidity were age and region, they varied depending on the type of multimorbidity. Therefore, surveillance of type of 
multimorbidity in clinical care should routinely be conducted to properly decide the treatment and follow-up care among older patients with late-stage melanoma. 
Table 2.1

Percent with Immune Checkpoint Inhibitor (ICI) use by Selected Patient-level Characteristics among Older Adults (age > 65 years) with Incident Late-stage Melanoma during 2012 and 2015

Linked Surveillance, Epidemiology, and End Results and Medicare Claims Database

\begin{tabular}{|c|c|c|c|c|}
\hline \multirow{2}{*}{ Variables } & \multicolumn{2}{|r|}{ No ICI } & \multirow[b]{2}{*}{ p-value } & \multirow[b]{2}{*}{ Significance } \\
\hline & $\mathbf{N}(\%)$ & $\mathbf{N}(\%)$ & & \\
\hline ALL & $252(5.6)$ & $4,267(94.4)$ & & \\
\hline \multicolumn{5}{|l|}{ Multimorbidity } \\
\hline Yes & $211(5.5)$ & $3,622(94.5)$ & 0.62 & \\
\hline No & $41(6.0)$ & $645(94.0)$ & & \\
\hline \multicolumn{5}{|l|}{ Year of diagnosis } \\
\hline 2012 & $35(3.2)$ & $1,060(96.8)$ & 0.0008 & $* * *$ \\
\hline 2013 & $63(5.8)$ & $1,026(94.2)$ & & \\
\hline 2014 & $74(6.4)$ & $1,090(93.6)$ & & \\
\hline 2015 & $80(6.8)$ & $1,091(93.2)$ & & \\
\hline \multicolumn{5}{|l|}{ Age } \\
\hline 66 to 69 years & $62(6.8)$ & $851(93.2)$ & 0.0004 & $* * *$ \\
\hline 70 to 74 years & $76(7.6)$ & $929(92.4)$ & & \\
\hline 75 to 79 years & $45(5.0)$ & $860(95.0)$ & & \\
\hline$\geq 80$ years & $69(4.1)$ & $1,627(95.9)$ & & \\
\hline \multicolumn{5}{|l|}{ Sex } \\
\hline Female & $72(4.5)$ & $1,545(95.5)$ & 0.014 & $*$ \\
\hline Male & $180(6.2)$ & $2,722(93.8)$ & & \\
\hline \multicolumn{5}{|l|}{ Race } \\
\hline Whites & $239(5.5)$ & $4,103(94.5)$ & 0.29 & \\
\hline Non-Whites & $13(7.3)$ & $164(92.7)$ & & \\
\hline \multicolumn{5}{|l|}{ Marital Status } \\
\hline Married & $163(7.3)$ & $2,057(92.7)$ & $<0.0001$ & $* * *$ \\
\hline Not married & $89(3.9)$ & $2,210(96.1)$ & & \\
\hline \multicolumn{5}{|c|}{ Dual Medicare/Medicaid eligibility } \\
\hline Yes & $17(10.0)$ & $153(90.0)$ & 0.0104 & $*$ \\
\hline No & $235(5.4)$ & $4,114(94.6)$ & & \\
\hline \multicolumn{5}{|l|}{ Regions } \\
\hline Northeast & $64(7.9)$ & $748(92.1)$ & 0.0186 & $*$ \\
\hline South & $49(5.1)$ & $916(94.9)$ & & \\
\hline North Central & $24(5.1)$ & $451(94.9)$ & & \\
\hline West & $115(5.1)$ & $2,152(94.9)$ & & \\
\hline
\end{tabular}

Note: Based on 4,519 older adults with incident late-stage (Stage III/IV) melanoma continuously enrolled in Medicare Part A \& B fee-for service programs 12 months prior to incident cancer diagnosis. $* 0.05<\mathrm{p} \leq 0.01 ; * * 0.01<\mathrm{p} \leq 0.001 ; * * * \mathrm{p}<0.001$ 
Table 2.2

Percent with Type of Multimorbidity by Selected Patient-level Characteristics Older Adults (age $\geq 65$ years) with Incident Late-stage Melanoma during 2011 and 2015 Linked Surveillance, Epidemiology, and End Results and Medicare Claims Database

\begin{tabular}{|c|c|c|c|c|c|c|}
\hline & \multicolumn{2}{|c|}{ PHC Multimorbidity } & \multicolumn{2}{|c|}{ AD Multimorbidity } & \multicolumn{2}{|c|}{ MHC Multimorbidity } \\
\hline & $\%$ Yes & $\%$ No & $\%$ Yes & $\%$ No & $\%$ Yes & $\%$ No \\
\hline \multicolumn{7}{|l|}{ Year of diagnosis } \\
\hline 2012 & 83.7 & 16.3 & 10.4 & 89.6 & 5.0 & 95.0 \\
\hline 2013 & 82.8 & 17.2 & 9.6 & 90.4 & 5.1 & 94.9 \\
\hline 2014 & 85.8 & 14.2 & 9.1 & 90.9 & 6.4 & 93.6 \\
\hline 2015 & 83.0 & 17.0 & 10.7 & 89.3 & 6.9 & 93.1 \\
\hline \multicolumn{7}{|l|}{ Age $^{a}$} \\
\hline 65 to 69 years & 76.0 & 24.0 & 8.8 & 91.2 & 6.6 & 93.4 \\
\hline 70 to 74 years & 81.9 & 18.1 & 9.6 & 90.4 & 5.8 & 94.2 \\
\hline 75 to 79 years & 85.2 & 14.8 & 9.8 & 90.2 & 6.0 & 94.0 \\
\hline$\geq 80$ years & 88.6 & 11.4 & 10.9 & 89.1 & 5.5 & 94.5 \\
\hline \multicolumn{7}{|l|}{$\operatorname{Sex}^{b, c}$} \\
\hline Female & 84.2 & 15.8 & 13.2 & 86.8 & 8.8 & 91.2 \\
\hline Male & 83.7 & 16.3 & 8.1 & 91.9 & 4.2 & 95.8 \\
\hline \multicolumn{7}{|l|}{ Race } \\
\hline White & 83.9 & 16.1 & 10.1 & 89.9 & 5.9 & 94.1 \\
\hline Non-Whites & 83.1 & 16.9 & 6.8 & 93.2 & 5.6 & 94.4 \\
\hline \multicolumn{7}{|l|}{ Marital status $^{c}$} \\
\hline Married & 84.2 & 15.8 & 10.4 & 89.6 & 3.9 & 96.1 \\
\hline Not married & 83.6 & 16.4 & 9.5 & 90.5 & 7.8 & 92.2 \\
\hline \multicolumn{7}{|l|}{ Region $^{\mathbf{a}, \mathbf{b}}$} \\
\hline Northeast & 87.4 & 12.6 & 11.9 & 88.1 & 6.3 & 93.7 \\
\hline South & 85.7 & 14.3 & 7.2 & 92.8 & 6.0 & 94.0 \\
\hline North central & 82.5 & 17.5 & 13.5 & 86.5 & 8.0 & 92.0 \\
\hline West & 82.1 & 17.9 & 9.7 & 90.3 & 5.2 & 94.8 \\
\hline \multicolumn{7}{|c|}{ Dual Medicare/Medicaid Enrollment ${ }^{\text {c }}$} \\
\hline Yes & 86.5 & 13.5 & 9.4 & 90.6 & 17.1 & 82.9 \\
\hline No & 83.8 & 16.2 & 10.0 & 90.0 & 5.4 & 94.6 \\
\hline
\end{tabular}

Note: Based on 4,519 older adults with incident late-stage (Stage III/IV) melanoma, continuously enrolled in Medicare Parts A and B fee-for service programs 12 months prior to incident cancer diagnosis.

AD: Autoimmune diseases; MHC: Mental health conditions; PHC: Physical health conditions

a represents significant group differences in presence or absence of PHC multimorbidity; ${ }^{\mathbf{b}}$ represents significant group differences in presence or absence of AD multimorbidity; c represents significant group differences in presence or absence of MHC multimorbidity based on chi-square tests. 
Table 2.3

Adjusted Odds Ratios (AOR) and 95\% Confidence Intervals (CI) from

Separate Multivariable Logistic Regressions on Type of Multimorbidity

Older Adults (age $\geq 65$ years) with Incident Late-stage Melanoma during 2011 and 2015

Linked Surveillance, Epidemiology, and End Results and Medicare Claims Database

\begin{tabular}{|c|c|c|c|c|c|c|c|c|c|}
\hline & \multicolumn{3}{|c|}{ PHC Multimorbidity } & \multicolumn{3}{|c|}{ AD Multimorbidity } & \multicolumn{3}{|c|}{ MHC Multimorbidity } \\
\hline & AOR & $95 \% \mathrm{CI}$ & Prob & AOR & $95 \% \mathrm{CI}$ & Prob & AOR & $95 \% \mathrm{CI}$ & Prob \\
\hline \multicolumn{10}{|l|}{ Year of diagnosis } \\
\hline 2013 & 0.95 & {$[0.76,1.19]$} & & 0.88 & {$[0.67,1.17]$} & & 0.75 & {$[0.53,1.08]$} & \\
\hline 2014 & 1.21 & {$[0.96,1.52]$} & & 0.82 & {$[0.62,1.08]$} & & 0.96 & {$[0.69,1.34]$} & \\
\hline \multicolumn{10}{|l|}{ Age } \\
\hline 65 to 69 years & [ref] & & & [ref] & & & [ref] & & \\
\hline 70 to 74 years & 1.42 & {$[1.13,1.77]$} & 0.002 & 1.11 & {$[0.81,1.52]$} & & 0.92 & {$[0.63,1.35]$} & \\
\hline 75 to 79 years & 1.81 & {$[1.42,2.30]$} & $<0.001$ & 1.16 & {$[0.84,1.59]$} & & 0.95 & {$[0.65,1.40]$} & \\
\hline Male & [ref] & & & [ref] & & & [ref] & & \\
\hline \multicolumn{10}{|l|}{ Race } \\
\hline White & [ref] & & & [ref] & & & [ref] & & \\
\hline Non-Whites & 1.03 & {$[0.68,1.55]$} & & 0.62 & {$[0.34,1.14]$} & & 0.63 & {$[0.32,1.24]$} & \\
\hline \multicolumn{10}{|l|}{ Marital status } \\
\hline Married & [ref] & & & [ref] & & & [ref] & & \\
\hline Not married & 0.91 & {$[0.77,1.07]$} & & 0.79 & {$[0.65,0.97]$} & 0.03 & 1.85 & {$[1.41,2.43]$} & $<0.001$ \\
\hline \multicolumn{10}{|l|}{ Region } \\
\hline Northeast & [ref] & & & [ref] & & & [ref] & & \\
\hline
\end{tabular}

Note: Based on 4,519 older adults with incident late-stage (Stage III/IV) melanoma continuously enrolled in Medicare Parts A and B fee-for-service programs 12 months prior to incident cancer diagnosis.

AD: Autoimmune diseases; MHC: Mental health conditions; PHC: Physical health conditions; Prob: Probability 


\section{CHAPTER 3}

\section{FACTORS ASSOCIATED WITH IMMUNE CHECKPOINT INHIBITOR USE}

AMONG OLDER PATIENTS WITH LATE-STAGE MELANOMA

\subsection{Abstract}

Background: Improvement in overall survival by immune checkpoint inhibitors (ICI) treatment in clinical trials encourages their use for late-stage melanoma. However, in the realworld, heterogeneity of population, such as elderly with multimorbidity, may lead to a slower diffusion of ICIs. The objective of this study was to examine the association of multimorbidity and other factors to ICI use among older patients with late-stage melanoma using real world data.

Methods: A retrospective cohort study design with a 12-month baseline and follow-up period was adopted with data from the linked Surveillance, Epidemiology, and End Results cancer registry/Medicare database. Older patients ( $>65$ years) with late-stage (stage III/IV) melanoma diagnosed between 2012 and 2015 were categorized as with or without multimorbidity (presence of two or more chronic conditions) and ICI use was identified in the post-index period. Chi-square tests and logistic regression were used to evaluate factors associated with ICI use.

Results: In the study cohort, $85 \%$ had multimorbidity, $18 \%$ received any treatment (chemotherapy, radiation, and/or ICI), and 6\% received ICI. Only 5.5\% of older patients with multimorbidity and $6 \%$ without multimorbidity received ICIs. Younger age, presence of social support, lower economic status, residence in northeastern regions, and recent year of diagnosis were significantly associated with ICI use; however, multimorbidity, sex, and race were not associated with ICI use. 
Conclusions: In the real-world clinical practice, only one in 18 older adults with late stage melanoma received ICI, suggesting slow pace of diffusion of innovation. However, multimorbidity was not a barrier to ICI use.

\subsection{Introduction}

Newer therapies, namely immune checkpoint inhibitors (ICIs) with a unique mechanism of action and unknown side effect profile,${ }^{92}$ have significantly increased the survival prognosis for adults with late-state melanoma. ${ }^{19,22,93}$ The first ICI was approved in 2011 by the United States (US) Food and Drug Administration and has since been recommended as the first-line treatment for late-stage melanoma by the National Cancer Comprehensive Network (NCCN) guidelines. ${ }^{42,93}$ These recommendations were based on the evidence presented in randomized clinical trials (RCTs), which have strict inclusion and exclusion criteria. ${ }^{19,20,72}$ These stringent criteria, while beneficial to ensure patient safety, do not capture the heterogeneity of various patient subpopulations.

This lack of information on heterogeneity of treatment effects may be a reason that despite being around for nearly a decade, the uptake of ICI in the real-world setting is dismal. ${ }^{94}$ One of the patient subpopulations where evidence on use of ICI is lacking is the elderly. Although utility of ICI in elderly patients with late-stage melanoma is debated, data from studies have shown that these therapies are well tolerated in the elderly. ${ }^{95-97}$ Older patients are also known to have multiple chronic conditions (also known as multimorbidity), which are often not taken into account by the guidelines. ${ }^{95,96,98}$ There are no studies to-date examining the association of multimorbidity on treatment with ICI among older individuals with late-stage melanoma. Presence of multimorbidity leads to less aggressive treatment with existing modalities (such as chemotherapy and radiation) due to fear of worsening other conditions..$^{32,33,35}$ 
Older patients with multimorbidity are the norm rather than exception in clinical practices. ${ }^{29}$ Therefore, evaluating the association of multimorbidity to ICI use in real-world setting may help healthcare providers personalize these treatments for their older patients.

In addition, disparities in the receipt of ICI are unknown. Studies on other cancers have shown some subgroups may be less likely to receive treatment. For example, age and racial disparities on treatment received was reported among older patients with late-stage pancreatic adenocarcinoma. ${ }^{46}$ Patients below the age of 80 years and White non-Hispanics were more likely to receive treatment than those above 80 years of age and other race/ethnicity. ${ }^{46}$ Similarly, a study among older patients with bladder cancer reported better survival among married patients than unmarried ones, because of greater likelihood of receiving treatment. ${ }^{47}$ Underinsured patients with late-stage melanoma were more likely to receive treatment at lower immunotherapy prescribing hospitals. ${ }^{48}$ With evident disparities in receipt of treatment, it is critical to know whether some subgroups lag in the diffusion of innovative therapies like ICIs, so that oncologists and patients alike can make informed decisions when considering ICIs as the treatment option. Therefore, the objective of this study is to examine the association of multimorbidity and other factors to ICI use that cover years from the initial introduction (i.e. 2011) to 2015 among older patients with late-stage melanoma.

\subsection{Methods}

\section{Study Design}

This was a retrospective cohort design with a 12-month baseline (pre-diagnosis) and 12-month follow-up (post-diagnosis) period. Diagnosis date of late-stage (stage III/stage IV) melanoma diagnosis was used to define pre- and post-diagnosis periods. Multimorbidity and all 
independent variables were assessed in the baseline period while treatments (chemotherapy, radiation, ICI) received were assessed in the follow-up period.

\section{Data Source}

This study was conducted using the Surveillance, Epidemiology, and End Results (SEER) cancer registry linked with fee-for-service Medicare claims. Information on clinical variables related to cancer (such as stage of cancer at diagnosis) was obtained from SEER data, while information on healthcare encounters of beneficiaries when enrolled and using Medicare covered health services was obtained from Medicare claims.

\section{Study Population}

Incident melanoma diagnosis between 2011 and 2015 was identified using ICD-O-3 site codes (C44.0 - C44.9) and ICI-O-3 histology codes (8720 - 8790). Late-stage (stage III/IV) of melanoma was identified based on the TNM classification using American Joint Committee on Cancer $7^{\text {th }}$ Edition. The final cohort comprised of 4,519 patients with late-stage melanoma following exclusion of those with local or regional (stage I/II) melanoma, non-incident melanoma, ages 66 years and below, not continuously enrolled in Medicare part A and part B during pre-index period, and diagnosed with late-stage cancer during autopsy.

\section{Measures}

\section{Dependent variable: ICI use}

The study outcome, ICI use, was identified in the post-diagnosis period. The ICIs approved for late-stage melanoma treatment include ipilimumab, nivolumab, and pembrolizumab, which were identified using healthcare common procedure coding system (HCPCS) codes (J9228, J9299, J9271). 


\section{Independent variables}

Multimorbidity: Presence of two or more chronic conditions in the pre-diagnosis period was defined as multimorbidity in this study. These conditions were obtained from a list of 18 chronic conditions developed by Multiple Chronic Conditions working group within the US Department of Health and Human Services Office of Assistant Secretary of Health. Pre-existing autoimmune diseases were also added to the list based on the current challenges with ICI use in patients with these conditions. ${ }^{73}$ All these conditions were identified with International Classification of Diseases (ICD), $9^{\text {th }}$ Edition.

Treatment with chemotherapy and radiation was also determined in the post-index period. Chemotherapy and radiation claims were identified using procedure codes, HCPCS codes, and revenue center codes (see Appendix 7.2). Overlapping procedure codes for chemotherapy and ICI $(96413,96415)$ were excluded to avoid confusion.

Biological factors consisted of age (66-69 years, 70-74 years, $75-79$ years, and $\geq 80$ years), sex (male/female), and race (white/non-white). Social factors included marital status (married/not married). Community resources included regions (Northeast, South, West, and North Central). Dual Medicare/Medicaid enrollment (yes/no) was used as a proxy for low economic status. Years of incident melanoma diagnosis (2012-2015) was used to control for changes in practice patterns.

\section{Statistical Analysis}

Chi-square tests were used to identify significant unadjusted associations of individual characteristics to ICI use. Multivariable Logistic regressions were performed to determine the association of multimorbidity, year of diagnosis, age, sex, race/ethnicity, marital status, dual eligibility, and region with ICI use. Parameter estimates are presented as adjusted odds ratios 
(AORs) after adjusting for all independent variables, with their corresponding 95\% confidence intervals (CI); $\mathrm{p} \leq 0.05$ was considered statistically significant. All analyses were conducted using SAS 9.4 (SAS Institute Inc.).

\subsection{Results}

The study population comprised predominantly of males (64.2\%), Non-Hispanic Whites (96.1\%) and those 70 years or older (70.4\%). About $85 \%$ of the older adults had multimorbidity, $18 \%$ received any treatment, and $6 \%$ received ICI. In the study cohort, $5.5 \%$ of patients with multimorbidity and $6 \%$ of patients without multimorbidity received ICI. The characteristics of those who received and did not receive ICI differed by all variables except the presence of multimorbidity $(\mathrm{p}=0.62)$ and race $(\mathrm{p}=0.29)$ (Table 3.1).

Factors associated with ICI use: Unadjusted Logistic Regression on ICI use

Table 3.2 presents the unadjusted odds ratio (OR), AORs, and 95\% CIs of all independent variables included in the study from separate logistic regressions on ICI use. In the unadjusted regressions, year of diagnosis, age, sex, marital status, and dual eligibility were significantly associated with ICI use.

Factors associated with ICI use: Multivariable Logistic Regression on ICI use

Patients in the lower age groups (66 to 69 years and 70 to 74 years) had significantly higher odds of receiving ICIs than those 80 years and above $(\mathrm{AOR}=1.65,95 \% \mathrm{CI}=1.15,2.36$; $\mathrm{AOR}=1.81,95 \% \mathrm{CI}=1.28,2.54$ respectively). Patients who were married $(\mathrm{AOR}=1.92$, $95 \% \mathrm{CI}=1.46,2.52)$, resided in the Northeastern SEER regions (AOR=1.75, 95\%CI=1.26, 2.41), and had dual eligibility ( $\mathrm{AOR}=2.42,95 \% \mathrm{CI}=1.40,4.19)$ were more likely to receive ICIs than the comparison groups: those who were not married, residing in SEER Western regions, and did 
not have dual eligibility. Patients diagnosed in 2012 ( $\mathrm{AOR}=0.42,95 \% \mathrm{CI}=0.28,0.63)$ were less likely to receive ICI than those diagnosed in 2015.

Multimorbidity was not significantly associated with ICI in fully adjusted models. In the fully adjusted model, sex and race were not significantly associated with ICI use.

\subsection{Discussion}

The treatment landscape for late-stage melanoma remained unchanged for decades before the introduction of ICIs in 2011. The median overall survival with traditional treatments (chemotherapy and/or radiation) is 6 to 8 months. ${ }^{99,100}$ Due to poor prognosis for survival, many patients may not receive treatment, as observed in this study. An overwhelming majority (82\%) of the cohort did not receive any treatment for their late-stage melanoma. Post-late stage melanoma diagnosis treatment rates (18\%) observed in this study is consistent with a published study $(22 \%) .{ }^{101}$ In this published study, the authors noted $22 \%$ initiated treatment after the disease progression while $51 \%$ started treatment before late-stage melanoma diganosis. ${ }^{101}$

Although ICIs have been around for nearly a decade, evidence on the treatment pattern in the real world is just emerging. Recent studies exploring the real-world treatment patterns among all adults for late-stage melanoma reported that only $34-37 \%$ of the patients received ICI as the first-line treatment, despite the recommendation by the NCCN guidelines. ${ }^{25,102,103}$ The rates of treatment with ICI in our study is very low (6\%). A plausible reason for the low rate can be due to the differences in population studied. Our study focused on older adults with $85 \%$ having pre-existing multimorbidity who may be at high risk for poor survival prognosis. As

evidence is still emerging on the side effect profile of ICIs compared to existing modalities, ${ }^{104}$ oncologists may be cautious in using ICI among older patients with late-stage melanoma. 
This study observed that an overwhelming majority of patients had pre-existing multimorbidity, no different than other cancer types. ${ }^{32,105}$ As RCTs of ICIs typically exclude patients with multimorbidity, ${ }^{106}$ evidence on the association of multimorbidity to ICI use is not available. This is the first study to report the use of ICI among older patients with multimorbidity status. In this study, those with multimorbidity were as likely to receive ICI as those without, suggesting that multimorbidity was not a barrier in the receipt of ICI. While the rationale for this was not explored further, plausible reasons are discussed. Recent studies using SEER-Consumer Assessment of Healthcare Providers and Systems suggest that patients with multimorbidity and cancer have better communication with their providers and rated specialties better than those without multimorbidity. ${ }^{107}$ It has also been reported that elderly Medicare beneficiaries with multimorbidity are equally likely to trust their doctors for their care, ${ }^{108}$ suggesting that multimorbidity may not be a barrier to novel life-saving therapies.

This is the first study to the best of our knowledge that explored the factors associated with ICI use among the elderly with late-stage melanoma. In this study, compared to patients 80 years and older, those between the ages of 66 and 74 years were more likely to receive ICIs. However, published studies did not find additional adverse events or difference in overall survival in patients between 80 to 100 years versus those between 65 to 79 years, when treated with ICIs. ${ }^{96}$ Oncologists may exercise caution in active treatment of cancer among old-old (age $>80$ years), because side effects can occur more often and in greater severity in this age group. ${ }^{105,109,110}$ Furthermore, higher rates of pre-existing chronic conditions in this age group may also warrant cautious active cancer treatment. In our study, nearly $90 \%$ of those 80 years or older had pre-existing multimorbidity compared to only $82 \%$ among 65 to 79 years. Due to 
small cell sizes, we were unable to empirically test the interaction of old-old with multimorbidity on ICI use in our study.

In this study, social support, measured with the proxy (i.e. marital status), was significantly associated with ICI use. Presence of social support have shown lesser psychological distress among patients with cancer and more enthusiasm about getting treatment, even if the disease is terminal. ${ }^{11,112}$ Therefore, these patients are more accepting of newer treatments. It is plausible that shared decision making with the patient and their caregivers/support system may increase the use of novel therapies in real-world settings.

Regional variations were also observed in our study, with older patients residing in Northeastern SEER regions having higher rates of ICI use. The reasons for differential adoption of newer treatments across the US regions is complex. Although to date, no study has examined regional disparities in ICI use among late-stage melanoma patients, few reasons for regional disparities based on evidence from the adoption of new medical treatments and new technologies are speculated here. The US states with higher population density may also have greater number of highly skilled professionals. In addition, these states tend to have policies that provide more opportunities to capitalize on innovations and are more likely to adopt innovations faster. ${ }^{113,114}$ Based on these factors, states in the Northeastern region including New York, Connecticut, and Massachusetts, had the highest innovation scores compared to other US states. ${ }^{113}$ In addition, key opinion leaders, who also lead many RCTs, play an important role in the diffusion of innovation. ${ }^{115}$ A study reported that such opinion leaders were based in urban areas, most of them in the Northeastern regions such as New York City and Boston. ${ }^{115}$ These leaders encouraged use of innovative therapies in real-world settings. ${ }^{115}$ Healthcare providers in various US regions should, therefore, evaluate the political influences in driving their prescribing 
practices and work with local opinion leaders in finding ways to improve adoption of newer therapies among patients.

In this study, dual Medicaid/Medicare eligibility was positively associated with ICI use. Previous studies have reported that dually eligible beneficiaries are less likely than Medicareonly beneficiaries to receive prostate or breast cancer treatment. ${ }^{116}$ In a study of lung cancer patients, dual eligibility status was associated with longer duration of treatment. ${ }^{117}$ Recent studies on late-stage melanoma reported that patients with Medicaid were less likely to receive ICIs and those with Medicare were as likely to receive ICIs as patients with commercial insurance. ${ }^{118-120}$ Thus, the receipt of ICI may be driven more by Medicare than Medicaid. Year of diagnosis was significantly associated with ICI use, with those diagnosed in earlier years being less likely to use ICIs. This may be because of few completed RCTs at the time and only one ICI (ipilimumab) approved for the treatment before 2014. Therefore, the data to support the safety and efficacy of ICI had not been widely been disseminated. In addition, the diffusion of innovation takes substantial time. ${ }^{94}$ Rather than rely on communication of a medical innovation, most physicians adopted the innovation after watching their colleagues use them. ${ }^{121}$ This is especially true when contemplating use in populations excluded in the RCTs, ${ }^{121}$ such as older patients with multimorbidity. Although the use of ICI as first-line treatment in late-stage melanoma was added in NCCN guideline in 2012, studies on the use of ICI in real-world settings remain limited.

\subsection{Strengths and limitations}

The findings of this study should be interpreted considering its limitations. First, the reasons for not receiving any treatment in older patients was unknown. Though disparities in ICI use were observed, we are unable to evaluate whether these disparities are due to patient 
preferences or shared decision-making of providers and patients. Such information could direct healthcare providers on measures that can be taken to enhance the adoption of ICIs. Second, individual-level socioeconomic factors, such as education and income, which may be associated with ICI use, were not available to us. Third, information on severity of co-existing illnesses may have provided insights into whether the intake is low because of competing demands that may confer high mortality risk and may have precluded the use of ICI. Despite these limitations, the study has several strengths. No study to-date has focused on treatment of older adults with multimorbidity and late-stage melanoma. With a high prevalence, oncologists are bound to encounter such patients on a daily basis. This study provides oncologists with strong evidence on the current treatment landscape among older adults with multimorbidity. In addition, this study examined the factors associated with ICI use. In the era of personalized medicines, patient-level factors play a critical role in treatment decisions. This study sheds light on various factors that will help healthcare providers in reaching a successful treatment goal with their older patients.

\subsection{Conclusions}

The findings from this study suggest that despite evidence of improved survival benefits over chemotherapy, the adoption of ICI among older patients remain low. This study revealed disparities in ICI use even after five years since ICI approval and introduction in the US markets. However, multimorbidity was not a barrier to ICI use suggesting that future research is needed on low uptake of ICI in older patients with multimorbidity. 


\section{TABLES}

Table 3.1

Percent with Immune Checkpoint Inhibitor (ICI) use by Selected Patient-level Characteristics among Older Adults (age > 65 years) with Incident Late-stage Melanoma during 2012 and 2015

Linked Surveillance, Epidemiology, and End Results and Medicare Claims Database

\begin{tabular}{|c|c|c|c|c|}
\hline \multirow{2}{*}{ Variables } & ICI & \multicolumn{3}{|l|}{ No ICI } \\
\hline & $\%$ & $\%$ & p-value & Significance \\
\hline $\mathbf{A L L}$ & 5.6 & 94.4 & & \\
\hline \multicolumn{5}{|l|}{ Multimorbidity } \\
\hline Yes & 5.5 & 94.5 & 0.62 & \\
\hline No & 6.0 & 94.0 & & \\
\hline \multicolumn{5}{|l|}{ Year of diagnosis } \\
\hline 2012 & 3.2 & 96.8 & 0.0008 & $* * *$ \\
\hline 2013 & 5.8 & 94.2 & & \\
\hline 2014 & 6.4 & 93.6 & & \\
\hline 2015 & 6.8 & 93.2 & & \\
\hline \multicolumn{5}{|l|}{ Age } \\
\hline 66 to 69 years & 6.8 & 93.2 & 0.0004 & $* * *$ \\
\hline 70 to 74 years & 7.6 & 92.4 & & \\
\hline 75 to 79 years & 5.0 & 95.0 & & \\
\hline$\geq 80$ years & 4.1 & 95.9 & & \\
\hline \multicolumn{5}{|l|}{ Sex } \\
\hline Female & 4.5 & 95.5 & 0.014 & $*$ \\
\hline Male & 6.2 & 93.8 & & \\
\hline \multicolumn{5}{|l|}{ Race } \\
\hline Whites & 5.5 & 94.5 & 0.29 & \\
\hline Non-Whites & 7.3 & 92.7 & & \\
\hline \multicolumn{5}{|l|}{ Marital Status } \\
\hline Married & 7.3 & 92.7 & $<0.0001$ & $* * *$ \\
\hline Not married & 3.9 & 96.1 & & \\
\hline \multicolumn{5}{|c|}{ Dual Medicare/Medicaid eligibility } \\
\hline Yes & 10.0 & 90.0 & 0.0104 & $*$ \\
\hline No & 5.4 & 94.6 & & \\
\hline \multicolumn{5}{|l|}{ Regions } \\
\hline Northeast & 7.9 & 92.1 & 0.0186 & $*$ \\
\hline South & 5.1 & 94.9 & & \\
\hline North Central & 5.1 & 94.9 & & \\
\hline West & 5.1 & 94.9 & & \\
\hline
\end{tabular}

Note: Based on 4,519 older adults with incident late-stage (Stage III/IV) melanoma continuously enrolled in Medicare Part A \& B fee-for service programs 12 months prior to incident cancer diagnosis. $* 0.05<\mathrm{p} \leq 0.01 ; * * 0.01<\mathrm{p} \leq 0.001 ; * * * \mathrm{p}<0.001$ 
Table 3.2

Unadjusted Odds Ratio (OR), Adjusted Odds Ratios (AOR), and 95\% Confidence Intervals (CI) from Logistic Regressions on Immune Checkpoint Inhibitor use

Older Adults (age >65 years) with Incident Late-stage Melanoma during 2012 and 2015

Linked Surveillance, Epidemiology, and End Results and Medicare Claims Database

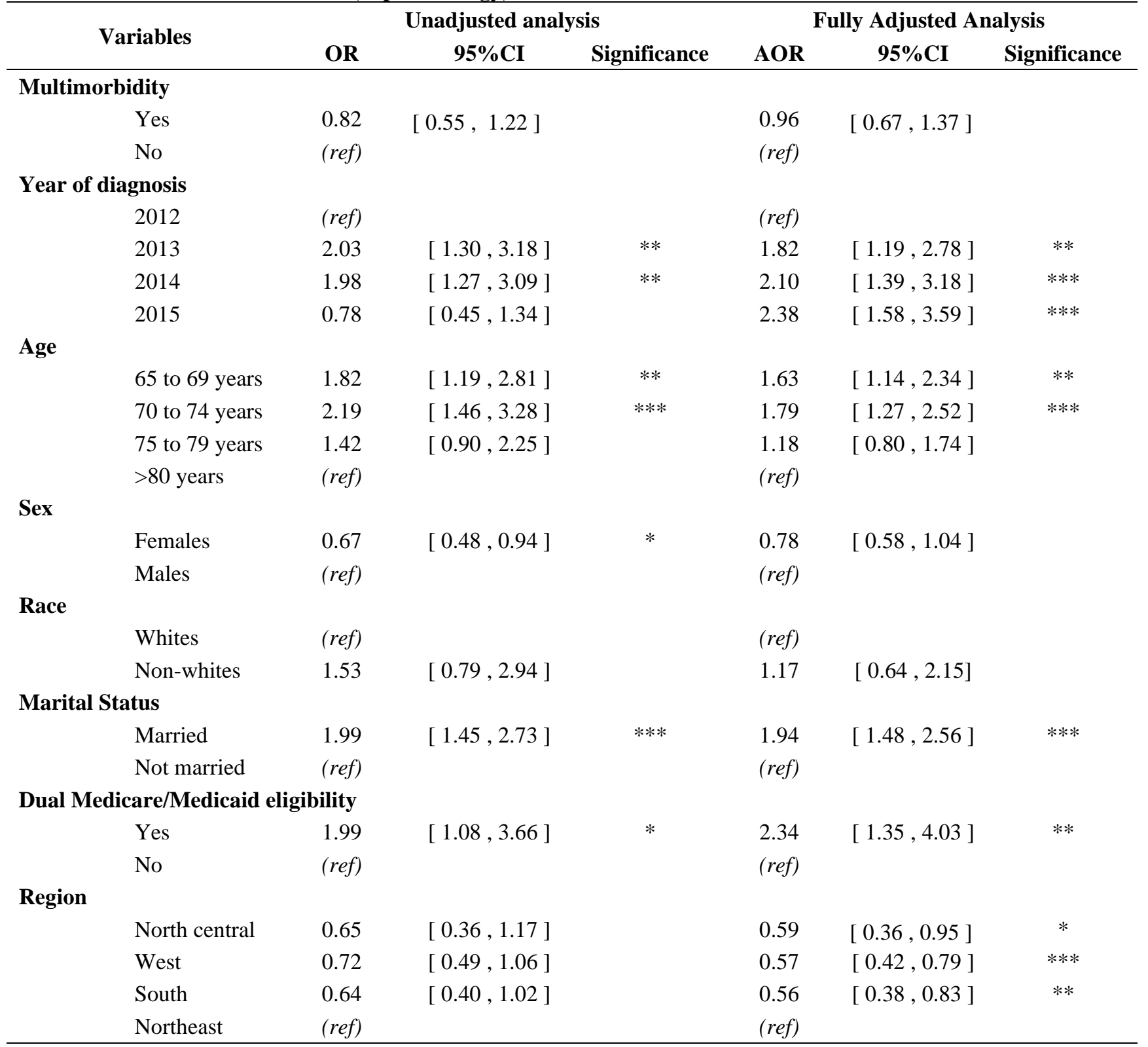

Note: Based on 4,519 older adults with incident late-stage (Stage III/IV) melanoma continuously enrolled in Medicare Parts A and B fee-for-service programs 12 months prior to incident cancer diagnosis. $* 0.05<\mathrm{p} \leq 0.01 ; * * 0.01<\mathrm{p}$ $\leq 0.001 ; * * * \mathrm{p}<0.001$

Abbreviations: ref $=$ Reference group 


\section{CHAPTER 4}

\section{IMMUNE CHECKPOINT INHIBITOR USE, MULTIMORBIDITY, AND HEALTHCARE EXPENDITURES AMONG OLDER ADULTS WITH LATE-STAGE MELANOMA}

\subsection{Abstract}

Background: The objective of this study is to assess the impact of immune checkpoint inhibitors (ICI) and multimorbidity on healthcare expenditures among older patients with late-stage melanoma.

Methods: A retrospective longitudinal cohort study using Surveillance, Epidemiology, and End Results linked with Medicare claims was conducted. Generalized linear mixed models were used to analyze adjusted relationships of ICI, multimorbidity, and ICI-multimorbidity interaction on average healthcare expenditures.

Results: Patients who received ICI and those who had multimorbidity had significantly higher average total healthcare expenditures compared to ICI nonusers and no multimorbidity. In the fully adjusted model using ICI-multimorbidity interaction, no excess cost was added by multimorbidity.

Conclusion: Use of ICIs, regardless of multimorbidity, is associated with increased healthcare expenditures.

\subsection{Introduction}

Cancer exerts a substantial burden on the morbidity and mortality of patients. ${ }^{122,123}$ In addition, cancer exerts a significant economic burden on not only the patients, but also on payers and society as well. ${ }^{50}$ Cancer is one of the top five most expensive chronic conditions. ${ }^{50}$ There is robust evidence showing that cancer substantially increases healthcare expenditures. ${ }^{124-127}$ For 
example, it has been reported that adults with cancer have four times higher expenditures compared to those without cancer. ${ }^{51,127}$ Furthermore, the healthcare cost of cancer differs by the type and stage of cancer. Healthcare costs were highest for cancers with poor survival rates such as brain cancers and lowest for cancers with high survival rates, such as melanoma. ${ }^{51}$ However, the cost increases substantially when the cancer metastasizes. ${ }^{50-52}$ For example, $55 \%$ of the annual direct costs for treating melanoma were related to treating late-stage melanoma. ${ }^{53}$

While many factors can influence healthcare costs among cancer patients, expensive drugs approved for cancer, the administration of these drugs, and supportive care (monitoring, surveillance, and management of side-effects such as kidney infections, pneumonia through frequent medical office visits and sometimes hospitalizations) are some of the main factors. ${ }^{56}$ For example, in metastatic melanoma patients initiated on an immune therapy agent ipilimumab, the average all-cause healthcare expenditures for a treatment episode was estimated to be $\$ 153,062 .{ }^{128}$ The immune checkpoint inhibitors (ICIs) were first approved for the treatment of late-stage melanoma nine years ago after their success in improving survival was established by randomized clinical trials (RCTs). ${ }^{92}$ However, the high cost of ICIs may hinder their widespread adoption in real-world clinical settings. The average wholesale price in 2015 of a single dose for a $70 \mathrm{~kg}$ patient was $\$ 5,732$ for nivolumab, $\$ 33,162$ for ipilimumab, and $\$ 35,073$ for the combination therapy. ${ }^{129}$ As these drugs need to be administered by healthcare professionals in outpatient settings, the total costs are even higher. ${ }^{130}$ This is compounded by the fact that an optimum dose for ICIs remains unknown which entails continuous use of ICI until regression of the tumor or appearance of adverse events. ${ }^{20,21,23}$ Oncologists estimated that treatment cost for the highest and most often administered dose, 26 courses of an ICI can be as high as $\$ 1,009,944$ with a $20 \%$ copay. $^{57}$ 
Thus, a vast majority of patients cannot afford ICI treatment unless covered by health insurance. ${ }^{48,120,131,132}$ In the United States (US), insurance and type of insurance coverage are often associated with prognosis, treatment and survival of cancer patients. Specifically, using the National Cancer Database, Jain and coauthors (2020) reported that the stage of diagnosis and receipt of ICIs were associated with insurance status. ${ }^{133}$ A high proportion of individuals with late-stage melanoma were on Medicare and were as likely as those with commercial insurance to receive ICI. ${ }^{48,49}$ These findings suggest that Medicare may bear a disproportionate share of latestage melanoma expenditures. Furthermore, older Medicare beneficiaries (age $>65$ years) are of particular interest for several reasons; $96 \%$ are covered by Medicare; higher incidence of better cancer/late-stage melanoma is observed among these patients, and Medicare patients are facing an evolving payment landscape, such as Oncology Care Model (OCM), aimed towards improving cancer care continuum while reducing costs in older patients. ${ }^{10,134-136}$

The newer payment models also include patients in high-risk groups, such as those with multimorbidity. ${ }^{136}$ This is because $68 \%$ of the Medicare population reported having multimorbidity and resulted in $80 \%$ of Medicare payments. ${ }^{29,137-139}$ Expert and systematic reviews have concluded that patients with multimorbidity have higher costs compared to those without multimorbidity. ${ }^{28,140}$ Patients with multimorbidity and cancer have higher expenditures compared to those without any multimorbidity. ${ }^{141,142}$ Furthermore, multimorbidity is highly prevalent in older adults with late-stage melanoma. ${ }^{143}$ If ICI are added to this mix, patients with multimorbidity and ICI may have even higher costs than those without multimorbidity and ICI. Therefore, the objectives of this study are (a) to estimate the impact of ICI use and (b) to assess the impact of the interaction of ICI and multimorbidity on healthcare expenditures among older patients with late-stage melanoma. We hypothesize that ICI use will be associated with high 
expenditures throughout the treatment period compared to those without ICI use, and those with multimorbidity and ICI will have even higher costs than those without multimorbidity and ICI.

\subsection{Methods}

\section{Study design}

A retrospective observational longitudinal cohort design with a 12-month baseline (preindex) and a 12-month follow-up (post-index) period was used; incident diagnosis of late-stage (stage III/IV) melanoma diagnosis was defined as the index date. Independent variables were assessed in the baseline period while treatments (chemotherapy, radiation, ICI) received were assessed in the follow-up period. Healthcare expenditures were measured every 120 days $\left(\mathrm{t}_{1}, \mathrm{t}_{2}\right.$, $t_{3}, t_{4}, t_{5}$, and $t_{6}$ ) during the 24 -month observation period to ensure robust findings by reducing the “signal-to-noise" ratio ${ }^{144}$ (Figure 4.1).

\section{Data Sources}

Surveillance, Epidemiology, and End Results (SEER) cancer registry linked with fee-forservice Medicare claims was used as the data source. Information on clinical variables related to cancer (such as stage of cancer at diagnosis) was obtained from the SEER data. Information on healthcare encounters of beneficiaries when enrolled and using Medicare covered health services including Medicare payments, and provider settings was obtained from Medicare claims.

\section{Study Population}

The study population was comprised of older ( $>65$ years) adults diagnosed with incident melanoma between 2012 and 2015, identified using ICD-O-3 site codes (C44.0 - C44.9) and ICI-O-3 histology codes $(8720$ - 8790). Late-stage (stage III/IV) of melanoma was identified based on the TNM classification using American Joint Committee on Cancer $7^{\text {th }}$ Edition. After excluding patients with local or regional (stage I/II) melanoma, non-incident melanoma, ages 
66 years and below, not continuously enrolled in fee-for-service Medicare part A and part B during the observation period, and diagnosed with late-stage cancer during autopsy, the final cohort consisted of 4,519 patients.

\section{Measures}

\section{Dependent variable: Total and Type of Healthcare expenditures}

Total healthcare expenditures consisted of the sum of Medicare payments for inpatient, outpatient services (including carrier claims) for any care, home health care, and durable medical equipment. We also analyzed type of healthcare expenditures by site of care (inpatient and outpatient, and home healthcare). All healthcare expenditures were adjusted by the Consumer Price Index for medical services ${ }^{145}$ and expressed in 2016 USD.

\section{Independent variables}

The use of ICI (yes/no) was identified in the post-diagnosis period. The three ICIs approved for late-stage melanoma treatment, ipilimumab, nivolumab, and pembrolizumab, were identified using healthcare common procedure coding system (HCPCS) codes (J9228, J9299, J9271). Overlapping procedure codes for chemotherapy and ICI $(96413,96415)$ were excluded to ensure that chemotherapy was not misclassified as ICI.

Multimorbidity (yes/no) was defined as the presence of two or more chronic conditions in in this study. These conditions were obtained from a list of 18 chronic conditions developed by Multiple Chronic Conditions working group within the US Department of Health and Human Services Office of Assistant Secretary of Health. ${ }^{41}$ Pre-existing autoimmune diseases were added to the list based on the current challenges with ICI use in patients with these conditions. ${ }^{42}$ All the chronic conditions were identified with International Classification of Diseases (ICD), 9th Edition. 
To examine the effects of ICI-multimorbidity interaction on cost, an ICI-multimorbidity interaction term was created, which was categorized into 4 groups: ICI and multimorbidity, ICI and no multimorbidity, No ICI and multimorbidity, and No ICI and no multimorbidity.

Other independent variables included biological factors, social factors, community resources, economic status, and year of diagnosis. Biological factors consisted of age (66-69 years, 70-74 years, 75-79 years, and $\geq 80$ years), sex (male/female), and race (white/non-white).

Social factors included marital status (married/not married). Oncologist visits (yes/no) and primary care physician visits (yes/no) were measured every 120 days. Dual Medicare/Medicaid enrollment (yes/no) was used as a proxy for low economic status. Years of incident melanoma diagnosis (2012-2015) was used to control for changes in practice patterns.

\section{Statistical Analyses}

Unadjusted subgroup differences in time-invariant characteristics between ICI users were tested with chi-square statistics. The associations of ICI and multimorbidity to healthcare expenditures were tested within the framework of Generalized Linear Models (GLM) with gamma distribution and log-link. This specification was chosen for GLM because of several reasons: (a) GLM does not require normal distribution of errors, (b) better aligns the variance function to the mean function, and (c) does not require smearing correction, which can be easily converted to original dollars. ${ }^{43}$ Modified Park test confirmed the choice of gamma distribution with log-link was selected. As healthcare expenditures were measured every 120 days during the pre- and post-index periods, each individual had six observations. However, these observations were not independent and therefore generalized linear mixed models (GLMMs) with gamma distribution and log-link was used to analyze adjusted relationships between ICI and non-ICI user groups and ICI-multimorbidity interaction. The GLMMs included all independent variables 
and time. Both unadjusted and adjusted models used GLMMs. Adjusted GLMMs included a time squared as one of the independent variables, to control for the non-linear relationship of healthcare expenditures over time. All analyses were conducted on STATA (StataCorp 2015).

\subsection{Results}

The study population was comprised of predominantly of males $(64.2 \%)$, Non-Hispanic Whites $(96.1 \%)$ and those 70 years or older (70.4\%). Most older patients with late-stage melanoma had multimorbidity $(85 \%)$ and $6 \%$ received ICI. The mean time from index date to ICI initiation was 48 days. The characteristics of the study population are presented in Appendix 7.3.

\section{Overall healthcare expenditures}

The average total, outpatient, home health, and inpatient healthcare expenditures in the pre-index period $\left(t_{1}, t_{2}\right.$, and $\left.t_{3}\right)$ were significantly $(\mathrm{p}<0.001)$ lower than the expenditures in the post-index period $\left(\mathrm{t}_{4}, \mathrm{t}_{5}\right.$, and $\left.\mathrm{t}_{6}\right)$ (Figure 4.2). All healthcare expenditures in the 120-day postlate-stage melanoma diagnosis period $\left(\mathrm{t}_{4}\right)$ were significantly higher compared to other pre- and post-index time periods $\left(\mathrm{t}_{1}, \mathrm{t}_{2}, \mathrm{t}_{3}, \mathrm{t}_{5}\right.$ and $\left.\mathrm{t}_{6}\right)$.

\section{Healthcare expenditures among ICI users and non-users}

The average total, outpatient, home healthcare, and inpatient healthcare expenditures were significantly higher among ICI users compared to non-ICI users. Among ICI users, the average total and inpatient expenditures were significantly higher in $\mathrm{t}_{4}$ (representing 120 days after cancer diagnosis) compared to other time periods $\left(t_{1}, t_{2}, t_{3}, t_{5}\right.$ and $\left.t_{6}\right)$ while average outpatient and home healthcare expenditures did not differ significantly in $t_{4}$ and $t_{5}$ and $t_{4}$ and $t_{6}$ time periods, respectively. Among non-ICI users, average total, outpatient, home healthcare, and inpatient expenditures were significantly higher in $t_{4}$ compared to other time periods. 


\section{Healthcare expenditures among those with and without multimorbidity}

The average total, outpatient, home healthcare, and inpatient healthcare expenditures were significantly higher among those with multimorbidity compared to those without multimorbidity. Compared to $t_{4}$, the average total, outpatient, home healthcare, and inpatient expenditures were significantly lower in other time periods $\left(t_{1}, t_{2}, t_{3}, t_{5}\right.$ and $\left.t_{6}\right)$ among those with multimorbidity. The average home healthcare expenditure did not significantly differ in $t_{4}$ and $t_{6}$ time periods among those without multimorbidity.

\section{Healthcare expenditures and ICI-multimorbidity interaction}

The average total, outpatient, home healthcare, and inpatient healthcare expenditures were significantly higher among ICI users and with multimorbidity group compared to ICI nonusers and without multimorbidity. Average total expenditures by the ICI-multimorbidity groups are displayed in Figure 4.3. Compared to $t_{4}$, the average total, outpatient, home healthcare, and inpatient expenditures were significantly lower in other time periods $\left(t_{1}, t_{2}, t_{3}, t_{5}\right.$ and $\left.t_{6}\right)$ among ICI users and with multimorbidity.

\section{Adjusted associations of ICI and multimorbidity to total healthcare expenditures}

Table 4.1 presents the unadjusted and adjusted parameters of GLMM on average total healthcare expenditures. The unadjusted and fully adjusted models showed similar results. In the model that was adjusted for time and time-squared, those with ICI had significantly higher average expenditures than those without ICI $(\beta=1.34, \mathrm{SE}=0.52, \mathrm{p}<0.001)$. When adjusted for multimorbidity, ICI users and those with multimorbidity had higher average expenditures compared to ICI non-users or no multimorbidity. In the fully adjusted model, the average total expenditure significantly increased with time $(\beta=0.49, \mathrm{SE}=0.04, \mathrm{p}<0.001)$. Patients who received ICI $(\beta=0.91, \mathrm{SE}=0.07, \mathrm{p}<0.001)$ and those who had multimorbidity $(\beta=0.72, \mathrm{SE}=0.07$, 
$\mathrm{p}<0.001)$ had significantly higher average total expenditures compared to those who did not receive ICI or did not have multimorbidity.

Other variables with significant findings were age, dual eligibility, and visits to oncologists and primary care physicians. Significantly higher average total expenditures were reported in patients aged 75 to 79 years $(\beta=0.17, \mathrm{SE}=0.07, \mathrm{p}=0.015)$ and 80 years and older $(\beta=0.26, \mathrm{SE}=0.06, \mathrm{p}<0.001)$ compared to those aged between 65 and 69 years, who were dual eligible $(\beta=0.28, \mathrm{SE}=0.11, \mathrm{p}=0.011)$ versus those who were not, who had visited oncologists ( $\beta=1.75, \mathrm{SE}=0.05, \mathrm{p}<0.001)$ versus no oncologist visits, and who had visited primary care physician $(\beta=1.38, \mathrm{SE}=0.05, \mathrm{p}<0.001)$ versus no primary care physician visits.

We also conducted GLMM on average total expenditures by ICI-multimorbidity interaction. In the fully adjusted model, compared to no ICI/no multimorbidity, average total expenditures were significantly higher in all other groups, namely in patients who used ICI and had multimorbidity $(\beta=17889.18, \mathrm{SE}=1260.794, \mathrm{p}<0.001)$, who used ICI but did not have multimorbidity $(\beta=16178.22, \mathrm{SE}=2951.45, \mathrm{p}<0.001)$, and who did not use ICI but had multimorbidity $(\beta=1510.35, \mathrm{SE}=368.74, \mathrm{p}<0.001)$. The contrast in average healthcare expenditures was examined by ICI use and multimorbidity by using ICI-no multimorbidity as the comparator group (Table 1). In the fully adjusted model, the average total healthcare expenditure significantly increased with time $(\beta=0.50, \mathrm{SE}=0.04, \mathrm{p}<0.001)$. Patients who were non-ICI users and had multimorbidity $(\beta=-0.73, \mathrm{SE}=0.14, \mathrm{p}<0.001)$ and non-ICI users and with no multimorbidity $(\beta=-1.51, \mathrm{SE}=0.15, \mathrm{p}<0.001)$ had significantly lower costs compared to patients who were ICI users and had no multimorbidity. However, there was no significant difference between patients who were ICI users and had multimorbidity and patients who were 
ICI users and had no multimorbidity. Other variables with significant findings were same as above.

\section{Adjusted relationships between ICI, multimorbidity, and type of healthcare expenditures}

The relationship between ICI and multimorbidity by type of expenditures (outpatient, home healthcare, and inpatient) was further explored. The findings for average outpatient expenditures were similar to total expenditures (Appendix 7.4).

While patient with multimorbidity had significantly higher average home healthcare and inpatient expenditures compared to those without multimorbidity, ICI use was not significantly associated with those expenditures (Appendix 7.5 and Appendix 7.6, respectively). Similarly, the unadjusted and adjusted models of the effect of ICI and multimorbidity interaction on average home healthcare and inpatient expenditures were similar. In the fully adjusted model, the average home healthcare $(\beta=0.19, \mathrm{SE}=0.06, \mathrm{p}=0.002)$ and inpatient expenditure $(\beta=1.56$, $\mathrm{SE}=0.22, \mathrm{p}<0.001)$ significantly increased with time. However, no significant difference was observed among the ICI and multimorbidity groups.

\subsection{Discussion}

This study confirms previous findings and also presents some new findings. First, this study reports that average healthcare expenditures significantly increased after a terminal cancer, i.e., late-stage melanoma, diagnosis, which is in line with published studies. Cancer diagnosis exerts a huge financial burden on patients, payers, and healthcare systems, with four times higher costs in cancer cohort compared to the non-cancer cohort. ${ }^{3,7}$ Late-stage cancers tend to be more expensive than early-stage cancers. ${ }^{4,8,44,45}$ Although costs of early-stage versus late-stage melanoma were not compared, these findings still relay that late-stage melanoma diagnosis among older patients places enormous burden on the payers. These findings have implications 
for the new OCM that are being experimented by the CMS. Under the OCM providers are expected to "provide higher quality, more highly coordinated oncology care at the same or lower cost to Medicare". ${ }^{29}$ The OCM organizes care around six-month episodes. ${ }^{29}$ By providing information on total healthcare expenditures by time periods, our cost estimates can serve as benchmarks for episode-driven payments.

Second, this study found that ICI use is associated with higher average healthcare expenditures among older Medicare beneficiaries with late-stage melanoma. This is the first study to the best of our knowledge to assess the association of ICI use on healthcare expenditures among older adults with late-stage melanoma. Despite having similar expenditures during the pre-index periods $\left(t_{1}, t_{2}\right.$, and $\left.t_{3}\right)$, use of ICI significantly increased post-index date $\left(t_{4}, t_{5}\right.$, and $\left.t_{6}\right)$ expenditures. As seen in our study, outpatient expenditures were the major drivers of healthcare expenditures, contributing to $83 \%$ of the total expenditures. This study also found that ICI use was not associated with inpatient expenditures. Taken together these findings suggest that healthcare costs of ICI users may be driven by supportive care. Reasons for these findings are speculated as follows. It is reported that administration costs associated with ICIs are higher compared to other therapies. ${ }^{15}$ Intravenous infusion of ICI requires constant monitoring by a healthcare professional, which may lead to higher healthcare utilization. ${ }^{46}$ Infusion reactions or immune-related adverse events may occur thereby increasing healthcare resource utilization. ${ }^{46}$ Another possible reason could be the constant monitoring and testing of patients in the outpatient settings being treated with ICI. ${ }^{46}$ Prior to and after infusion, late-stage melanoma patients are usually tested for serum biomarkers, enzyme level, and blood count. ${ }^{46}$ The study findings confirm that cancer-related treatments have shifted from inpatient to outpatient settings. ${ }^{45,47}$ 
Third, older Medicare beneficiaries with multimorbidity had higher average total expenditures compared to those without multimorbidity. Across a range of healthcare settings and population, disproportionately higher costs were accounted for in a small number of patients and multimorbidity was highly prevalent in those patients. ${ }^{48}$ A majority of older adults with latestage melanoma also had multimorbidity. ${ }^{38}$ Therefore, higher total expenditures among older adults with multimorbidity corroborates findings from other studies. ${ }^{6,36}$ Many alternatives to traditional healthcare practices and payment models are being considered to account for the complex need of patients with multimorbidity ${ }^{35}$ Accountable Care Organizations and patientcentered medical homes over traditional practice models and bundled payment of services, which is more patient-centric than disease-centric, are a few such efforts. ${ }^{35}$ Though there are numerous challenges to adopting such policies on a larger scale, ${ }^{35}$ future studies need to explore whether these emerging models are effective in providing value-based care at lower costs for cancer patients in general and late-stage cancer patients in particular.

Fourth, the ICI-multimorbidity interaction revealed that multimorbidity did not play a significant role in the increase of expenditures. Rather, the high expenditures were due to the use of ICI. Although multimorbidity was associated with higher average expenditures in this study, the expenditures due to ICI use supersede those expenditures. The possible reasons for the increased average expenditures with ICI use are discussed above. These results further strengthen the need for newer payment models such as OCM, which focus on reducing cost of cancer care.

\subsection{Strengths and Limitations}

The findings of this study should be interpreted considering its limitations. First, Part D costs were not included. This is because patients with multimorbidity will have higher 
prescription costs compared to those without multimorbidity. This may show even higher costs for patients with multimorbidity, which would be unrelated to cancer care. However, the current therapies for melanoma are covered under Part B. Therefore, melanoma-related treatment costs were adequately captured in this study. Second, information on severity of co-existing illnesses may have provided insights into cluster of conditions which may lead to higher healthcare expenditures. Third, the findings of this study cannot be generalized to all Medicare beneficiaries as the study population was limited to only those residing in SEER regions. Fourth, a 12-month follow-up period may not be adequately assessing the impact of various factors on healthcare expenditures. However, the mean overall survival for late-stage melanoma is approximately a year. Therefore, a 12-month follow-up period was chosen. Despite these limitations, the study has several strengths. This study adopted a longitudinal design and compared expenditures over time. Additionally, no study to-date has focused on impact of ICI on healthcare expenditures among older adults with multimorbidity and late-stage melanoma. This study provides payers with strong evidence on the influences of various factors on healthcare expenditures.

\subsection{Conclusion}

The results of this study illustrate that the healthcare expenditures were higher after a late-stage melanoma diagnosis. Use of ICIs and presence of multimorbidity were associated with higher expenditures. Outpatient expenditures contributed largely to the increase in total expenditures. However, the ICI-multimorbidity interaction revealed that ICI was the driving force behind the higher expenditures. Future studies are needed to explore healthcare utilization among older patients with multimorbidity and late-stage melanoma and explore its effect on healthcare expenditures. 


\section{FIGURE}

Figure 4.1: Schematic of the study design. Each individual was observed for 24 months with a 12-month pre-index and 12-month post-index period. Healthcare expenditures and selected independent variables were measured repeatedly every 120 days during the pre-index $\left(\mathrm{t}_{1}, \mathrm{t}_{2}\right.$, and $\left.t_{3}\right)$ and post-index $\left(t_{4}, t_{5}\right.$, and $\left.t_{6}\right)$ periods, yielding a total of six repeated measures for every individual.

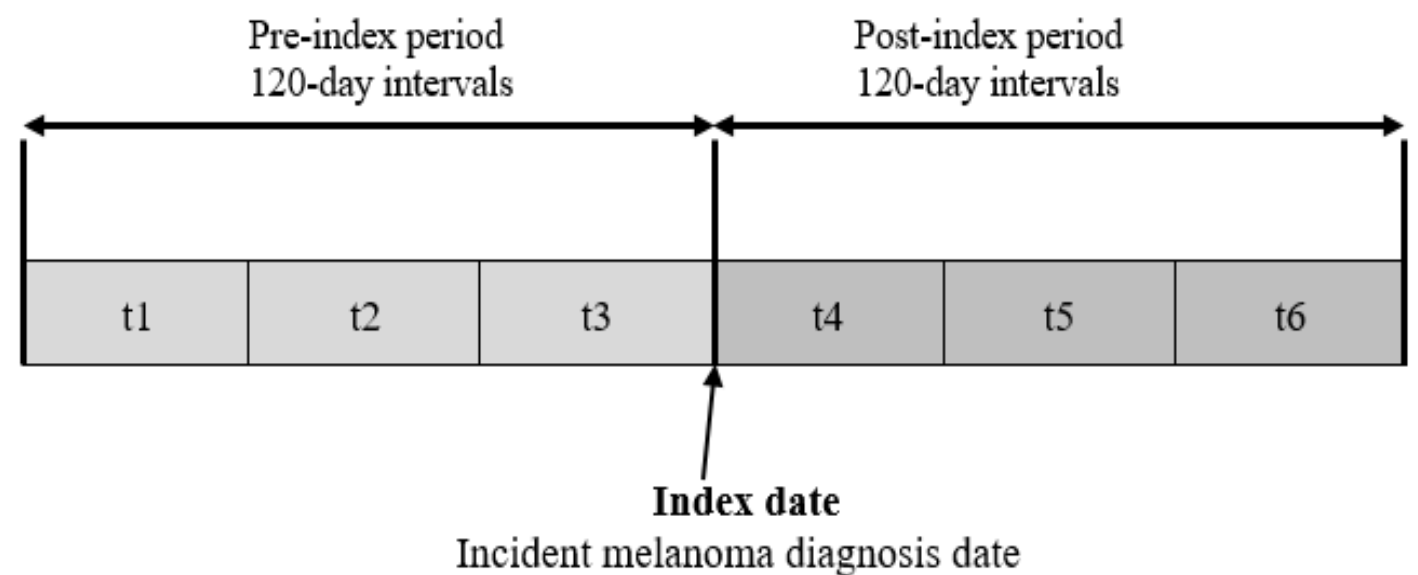




\section{FIGURE}

Figure 4.2: (a) Mean Total and Type of Healthcare Expenditures Over time; (b) Mean Total Healthcare Expenditures by ICI user status
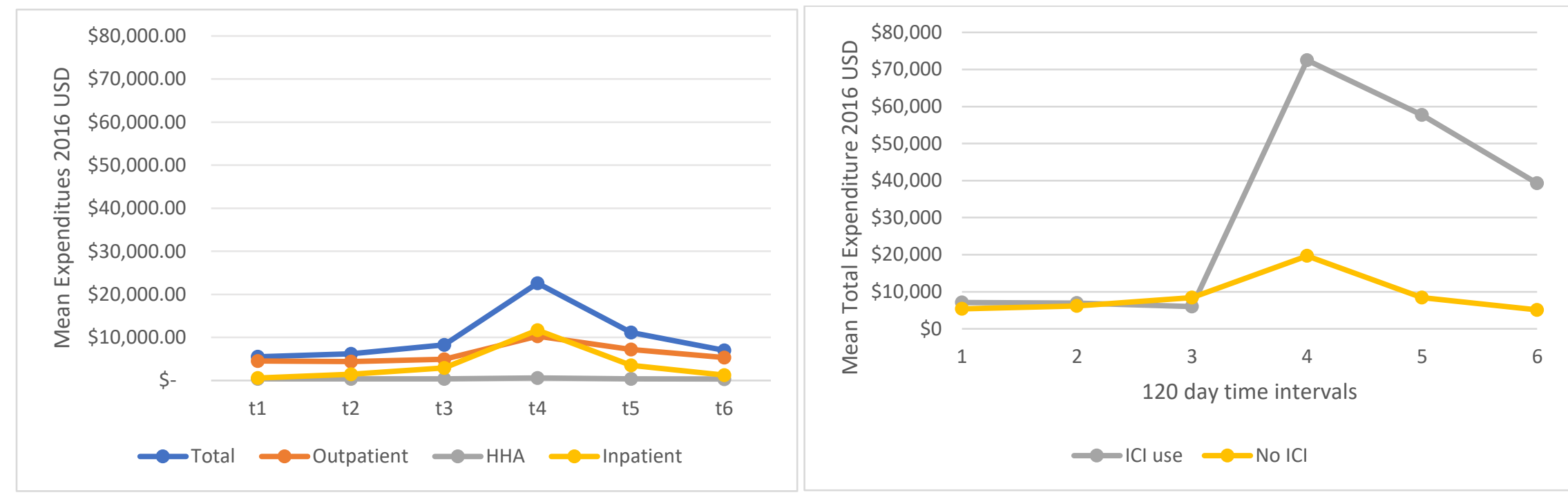

Based on 6 repeated observation of 4,519 older adults with incident late-stage (Stage III/IV) melanoma continuously enrolled in Medicare Parts A and $\mathrm{B}$ fee-for-service programs 12 months prior to incident cancer diagnosis.

Abbreviations: HHA, Home health care; ICI, Immune checkpoint inhibitors; USD, United States Dollars 


\section{FIGURE}

Figure 4.3: Mean total healthcare expenditures by ICI-Multimorbidity groups during pre-index and post-index periods

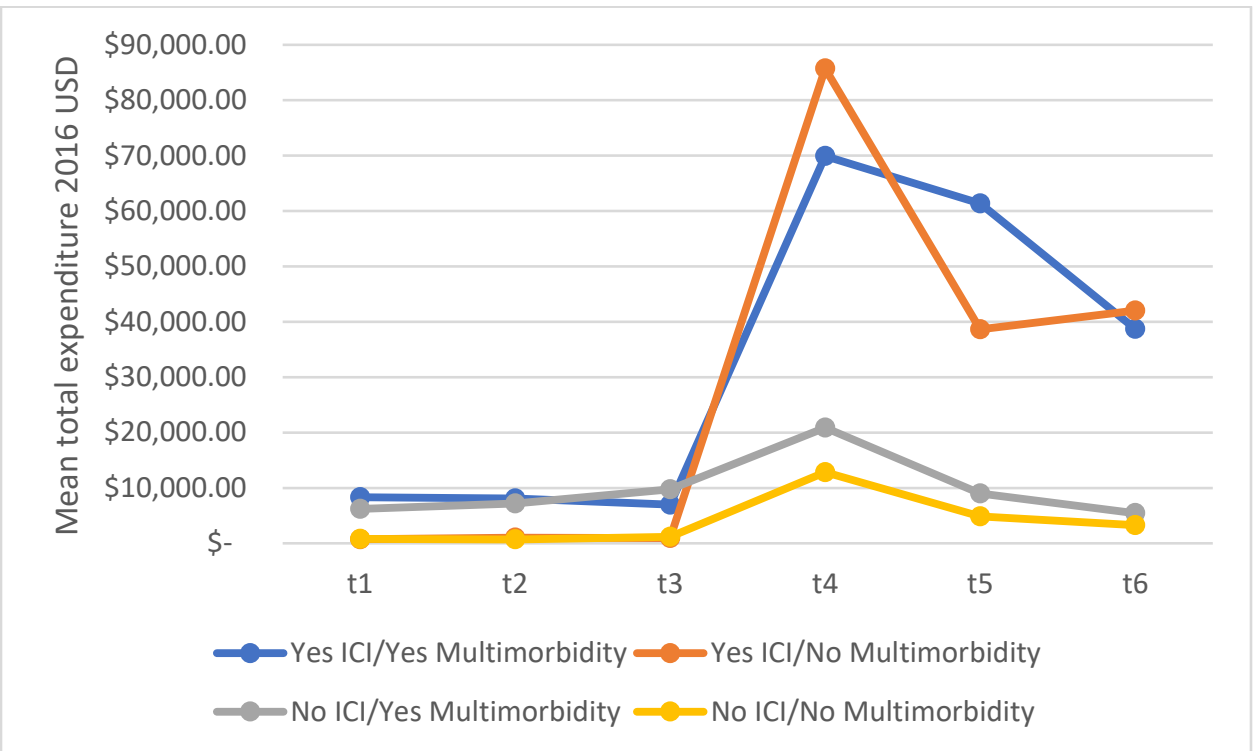

Based on 6 repeated observation of 4,519 older adults with incident late-stage (Stage III/IV) melanoma continuously enrolled in Medicare Parts A and B fee-for-service programs 12 months prior to incident cancer diagnosis. Abbreviations: ICI, Immune checkpoint inhibitors 
Table 4.1: Parameter estimates of select variables of GLMM on average total healthcare expenditures

\begin{tabular}{|c|c|c|c|c|c|c|}
\hline \multirow{3}{*}{ Variables } & \multicolumn{3}{|c|}{ No Interaction } & \multicolumn{3}{|c|}{ With interaction } \\
\hline & \multicolumn{6}{|c|}{ Unadjusted analysis } \\
\hline & Beta & SE & Prob & Beta & SE & Prob \\
\hline Time & 0.83 & 0.04 & $<0.001$ & 0.83 & 0.04 & $<0.001$ \\
\hline ICI use & & & & N/A & & \\
\hline Yes & 1.34 & 0.52 & $<0.001$ & & & \\
\hline No & $($ ref) & & & & & \\
\hline Multimorbidity & & & & N/A & & \\
\hline Yes & 0.96 & 0.74 & $<0.001$ & & & \\
\hline No & $($ ref) & & & & & \\
\hline ICI-Multimorbidity Interaction & N/A & & & & & \\
\hline Yes ICI and Yes Multimorbidity & & & & 0.19 & 0.10 & 0.051 \\
\hline Yes ICI and No Multimorbidity & & & & $(r e f)$ & & \\
\hline No ICI and Yes Multimorbidity & & & & -0.97 & 0.09 & $<0.001$ \\
\hline No ICI and No Multimorbidity & & & & -1.99 & 0.12 & $<0.001$ \\
\hline \multirow[t]{4}{*}{ Time squared } & -0.15 & 0.01 & $<0.001$ & -0.15 & 0.01 & $<0.001$ \\
\hline & \multicolumn{3}{|c|}{ No Interaction } & \multicolumn{3}{|c|}{ With interaction } \\
\hline & \multicolumn{6}{|c|}{$\begin{array}{l}\text { Fully adjusted analysis } \\
\end{array}$} \\
\hline & Beta & SE & Prob & Beta & SE & Prob \\
\hline Time & 0.49 & 0.04 & $<0.001$ & 0.50 & 0.04 & $<0.001$ \\
\hline ICI use & & & & N/A & & \\
\hline Yes & 0.91 & 0.07 & $<0.001$ & & & \\
\hline No & $(r e f)$ & & & & & \\
\hline Multimorbidity & & & & N/A & & \\
\hline Yes & 0.72 & 0.07 & $<0.001$ & & & \\
\hline No & (ref) & & & & & \\
\hline ICI-Multimorbidity Interaction & N/A & & & & & \\
\hline Yes ICI and Yes Multimorbidity & & & & 0.02 & 0.16 & 0.90 \\
\hline Yes ICI and No Multimorbidity & & & & $((r e f)$ & & \\
\hline No ICI and Yes Multimorbidity & & & & -0.73 & 0.14 & $<0.001$ \\
\hline No ICI and No Multimorbidity & & & & -1.51 & 0.15 & $<0.001$ \\
\hline Time squared & -0.12 & 0.01 & $<0.001$ & -0.12 & 0.01 & $<0.001$ \\
\hline
\end{tabular}

Note: Based on 6 repeated observation of 4,519 older adults with incident late-stage (Stage III/IV) melanoma continuously enrolled in Medicare Parts A and B fee-for-service programs 12 months prior to incident cancer diagnosis.

FFully adjusted model also included year of diagnosis, age, sex, race, marital status, dual eligibility, oncologist visit, and primary care physician visits.

Probability $\mathrm{p}<0.05$ was considered significant

Abbreviations: GLMM, Generalized linear mixed model; ICI, Immune checkpoint inhibitors; N/A, Not applicable; Prob, Probability; ref, Reference; SE, Standard error 


\section{CHAPTER 5}

\section{SUMMARY AND CONCLUSION}

\subsection{Summary of Findings and Discussion}

Pre-existing multimorbidity is prevalent among the elderly yet remains

understudied among older patients with melanoma. Patients with cancer often present with

multiple chronic conditions, i.e. multimorbidity ${ }^{65,66}$ Cancer is usually a manifestation of adverse lifestyles, such as smoking, obesity, and alcohol use, which are also the underlying causes of other chronic conditions. ${ }^{77}$ Therefore, as a person ages, they accumulate chronic conditions, thereby increasing the prevalence of multimorbidity in older ages, especially among the elderly (>65 years) patients with cancer. ${ }^{148}$ Despite being rampant in patients with cancer, multimorbidity is not included in cancer care. This is because patients with multimorbidity are often excluded from randomized clinical trials (RCTs).$^{72}$ Moreover, guidelines often adopt a "single-disease" approach, thereby posing a problem to oncologists on treating such patients. ${ }^{72}$ Information on prevalence of pre-existing multimorbidity may shed light on the need to update guidelines to include multimorbidity in cancer care.

While prevalence of multimorbidity in other cancers has been studied in detail, late-stage melanoma has not received wide attention. This may be because late-stage melanoma is the deadliest form of skin cancer with a high mortality rate. ${ }^{24}$ However, this was also the first cancer for which immune checkpoint inhibitors (ICI) were approved for treatment. ${ }^{92}$ The clinical trials of ICIs reported a significant improvement in the mean overall survival of patients with latestage melanoma, increasing from six to eight months to up to five years. ${ }^{93,149,150}$ As survivors of late-stage melanoma may increase, knowledge on the prevalence of pre-existing multimorbidity 
will assist healthcare providers personalize care for their older patients by creating guidelines with chronic conditions in mind.

\section{Prevalence of pre-existing multimorbidity among older adults with late-stage}

melanoma is high. This is the first study to explore the prevalence and factors associated with the presence and type of multimorbidity among older adults with late-stage melanoma in the US. This study found that pre-existing multimorbidity is highly prevalent among older adults with four out of five patients having two or more chronic conditions. This study also notes that the most common comorbid conditions were cardiovascular, endocrine, and musculoskeletal diseases or a combination of the three in dyads and triads. Cardiovascular, endocrine, and musculoskeletal diseases are highly prevalent in older adults. ${ }^{75,76}$ As these diseases and cancer share modifiable risk factors, with some studies stating a bidirectional relationship between them, our findings were not surprising. ${ }^{77-79}$ These findings point to the fact that healthcare providers managing patients with late-stage melanoma may need to routinely monitor for the presence of these conditions and coordinate their care with the primary care physicians.

Various types of multimorbidity (any, physical health conditions [PHC], autoimmune diseases $[\mathrm{AD}]$, and mental health conditions $[\mathrm{MHC}]$ multimorbidity) and the risk factors associated with those multimorbidity were also explored. This study confirms findings from previous studies that age is a risk-factor for any multimorbidity. ${ }^{29,33}$ The region of residence was also associated with multimorbidity; however, studies on regional variations are lacking for us to adequately explain these results. The PHC multimorbidity shared risk-factors with any multimorbidity, perhaps because PHC contributed to the bulk of multimorbidity. Pre-existing $\mathrm{AD}$ was one of the PHC focused on, because of its complicated relationship with ICIs and the surge of interest in this condition among the oncology community. This study found that 
although prevalence of pre-existing $\mathrm{AD}$ was not high, one in 10 older patients with late-stage melanoma will present with multimorbidity. Moreover, sex, region, and social support as a risk factor for AD multimorbidity also mimicked the findings of a previous studies. ${ }^{36,82,83}$ Oncologists should consider these risk factors when deciding if a drug acting via the immune system (such as ICI) is the best course of treatment for older patients. About $30 \%$ of older adults with late-stage melanoma in this study were diagnosed with any MHC, consistent with literature. ${ }^{84,85}$ The risk factors associated with MHC were sex, social support, and dual Medicare/Medicaid eligibility, which was consistent with previous studies. ${ }^{86-90}$ These risk factors with MHC should be assessed in order to reduce further psychological distress and appropriate follow-up interventions.

\section{The treatment landscape of late-stage melanoma has changed since the approval of}

\section{ICIs in 2011. However, it is unknown if these changes have been implemented to real-} world clinical settings. Despite the poor prognosis for survival, an overwhelming majority $(82 \%)$ of older patients in this study did not receive any treatment for their late-stage melanoma, which was consistent with a previous study on chemotherapy for late-stage melanoma treatment. ${ }^{101}$ In addition, the rates of treatment with ICI in the study was very low $(6 \%)$ and lower than that reported in recent studies..$^{25,102,103}$ The reason speculated was due to the differences in the population studied. The study also focused on older adults with $85 \%$ having pre-existing multimorbidity who may be at high risk for poor survival prognosis. As evidence is still emerging on the side effect profile of ICIs compared to existing modalities, ${ }^{104}$ oncologists may be cautious in using ICI among older patients with late-stage melanoma.

The factors associated with use of ICI among older patients with late-stage melanoma were determined to be lower age range, presence of social support in the form of a spouse, 
having dual Medicare/Medicaid eligibility, residing in Northeast region, and recent years of diagnosis. However, multimorbidity was not significantly associated with receipt of ICI suggesting that multimorbidity was not a barrier in the receipt of ICI. Since guidelines on cancer care do not take multimorbidity into consideration, these results entail that those lack of guidelines may force oncologists to use newer therapies despite concerns regarding additional conditions.

Healthcare expenditures in the United States are increasing. However, healthcare expenditures associated with ICI use and multimorbidity among older-adults with latestage melanoma remain unknown. This study reports that healthcare expenditures significantly increased after a terminal cancer, such as late-stage melanoma, diagnosis, which is in line with published studies. ${ }^{50-52,127}$ Older patients who had multimorbidity and those who had used ICI had significantly higher healthcare expenditures compared to those who did not have multimorbidity and had not use ICIs. Moreover, among ICI users, outpatient expenditures were the driving force behind total expenditures, accounting for $83 \%$ of the total expenditures. This confirmed findings from previous studies that late-stage melanoma care have shifted from inpatient to outpatient settings. ${ }^{54,147}$ In addition, the administration costs associated with ICIs are higher compared to other therapies. ${ }^{130}$ The ICI-multimorbidity interaction revealed that irrespective of the multimorbidity status, ICI was the driving force behind the total healthcare expenditures. This finding shows that expenditures associated with ICI use were higher than those accrued by multimorbidity. Taken together, the use of ICI is significantly associated with higher total healthcare expenditures. 


\subsection{Implications and suggestions for future research}

The findings of this study suggest that oncologists will encounter four in five older latestage melanoma patients with multimorbidity. With lack of late-stage melanoma treatment guidelines for older patients with multimorbidity, clinicians and other stakeholders should consider updating guidelines to better guide healthcare workers on treatment of such patients. The lack of clear evidence from clinical trials and guidelines may, therefore, leave oncologists guessing on treating older patients with newer therapies. With an already poor prognosis associated with late-stage melanoma, ICIs may be used regardless of multimorbidity.

Future studies exploring the outcomes, such as . overall survival, associated with ICI use for late-stage melanoma in older adults with multimorbidity may shed light on the effectiveness of ICI in this subpopulation. Further, exploring the effect of various dyad and triad clusters of chronic conditions on survival after ICI use may provide useful information on conditions highly susceptible to adverse outcomes after ICI use. For example, patients with pre-existing autoimmune diseases and cardiovascular diseases may not be good candidates for receiving ICI compared to patients with osteoporosis and depression. This is because conditions like cardiovascular diseases have intersecting pathways with cancer, as explained above, and therefore, are adversely affected by ICI further exacerbating these conditions. Health-related quality of life among ICI users and nonusers was not explored in this study. Future studies can explore the humanistic burden of ICI use among older adults with or without multimorbidity.

Healthcare expenditures were higher among ICI users, regardless of multimorbidity. The newer payment models like Oncology Care Models are needed now more than ever to reduce healthcare expenditures. This study provides policymakers, healthcare workers, and other 
stakeholders with a starting point for curbing the high expenditures among older patients with late-stage melanoma.

Future studies can further explore the healthcare utilization among these patients and the difference in healthcare expenditures between ICI users and non-users. Moreover, future studies can also include Part D expenditures. Prescription drug expenditures may be higher among patients with multimorbidity compared to those without multimorbidity. Therefore, the healthcare expenditures of older patients with multimorbidity may be even higher. These studies will strengthen the need for preventative measures to avoid multimorbidity among the elderly.

\subsection{Strengths and Limitations}

The present study has several limitations. First, SEER does not contain information on individual behavior such as smoking status, alcohol consumption, or functional status, all of which can further affect the risk of having multimorbidity. Second, individual-level socioeconomic factors, such as education and income were not available. Third, information on severity of co-existing illnesses may have provided insights into whether the intake is low because of competing demands that may confer high mortality risk and may have precluded the use of ICI. Fourth, the reasons for not receiving any treatment in older patients was unknown. Though disparities in ICI use were observed, the reason for these disparities, whether due to patient preferences or shared decision-making of providers and patients, could not be evaluated. Such information could direct healthcare providers on measures that can be taken to enhance the adoption of ICIs. Fifth, the database did not have detailed information on tumor characteristics or genetic markers such as BRAF status. Information on these could provide further insights into use of ICIs. Sixth, Part D medications were unavailable to us and therefore, prescription expenditure was not included. However, the current therapies for melanoma are covered under 
Part B. Therefore, melanoma-related treatment costs were adequately captured in this study. Seventh, a 12-month follow-up period may not be adequately assessing the impact of various factors on healthcare expenditures. However, the mean overall survival for late-stage melanoma is approximately a year. Therefore, a 12-month follow-up period was chosen.

Despite these limitations, this study has several strengths. Although few studies on comorbid conditions in individuals with melanoma exist, they did not focus on late-stage melanoma, perhaps because the prognosis was poor with low chances of survival. ${ }^{91}$ Therefore, prognostic burden of multimorbidity in such individuals was deemed less important. ${ }^{91}$ However, newer therapies such as ICIs will improve survivorship of patients with late-stage melanoma. Therefore, information on the prevalence and type of multimorbidity in such individuals can help both physicians and patients in choice of cancer treatment, survivorship plans, and quality of life care. An exhaustive list of PHC and MHC, including pre-existing AD was included. Moreover, no study to-date has focused on treatment of older adults with multimorbidity and late-stage melanoma. With a high prevalence, oncologists are bound to encounter such patients on a daily basis. This study provides oncologists with strong evidence on the current treatment landscape among older adults with multimorbidity. In addition, this study examined the factors associated with ICI use. In the era of personalized medicines, patient-level factors play a critical role in treatment decisions. This study sheds light on various factors that will help healthcare providers in reaching a successful treatment goal with their older patients. The study on healthcare expenditure adopted a longitudinal design and compared expenditures over time. The longitudinal design allowed us to minutely look at the various time points pre- and post- cancer diagnosis and evaluated the reasons for changing expenditures in during each time point. This 
study provides payers with strong evidence on the influences of various factors on healthcare expenditures.

\subsection{Conclusion}

This dissertation concludes that prevalence of multimorbidity among older adults with late-stage melanoma is high. However, multimorbidity is not a barrier to access to ICIs. Multimorbidity is associated with higher expenditures compared to no multimorbidity. However, when the patients were treated with ICI, the healthcare expenditures increased significantly and the presence or absence of multimorbidity did not change the results. Clinical trials have shown that the ICIs have drastically improved survival of patients with late-stage melanoma; however, they come with a hefty price tag. Future studies are needed to examine overall survival among older patients with multimorbidity treated with ICIs to assess the riskbenefit of these newer therapies for late-stage melanoma. 


\section{REFERENCES}

1. Ward WH, Farma JM, eds. Cutaneous Melanoma: Etiology and Therapy. Brisbane (AU); 2017. doi:10.15586/codon.cutaneousmelanoma.2017

2. Matthews NH, Li W-Q, Qureshi AA. Epidemiology of Melanoma. In: Ward W, Farma J, eds. Cutaneous Melanoma: Etiology and Therapy [Internet]. Brisbane (AU): Codon Publications; 2017. https://www.ncbi.nlm.nih.gov/books/NBK481862/ doi: 10.15586/codon.cutaneousmelanoma.2017.ch1.

3. Tuong W, Cheng LS, Armstrong AW. Melanoma: Epidemiology, Diagnosis, Treatment, and Outcomes. Dermatol Clin. 2012;30(1):113-124. doi:10.1016/j.det.2011.08.006

4. National Cancer Institute. Bethesda M. SEER Cancer Stat Facts: Melanoma of the Skin. SEER Cancer Statistics Review, 1975-2015. https://seer.cancer.gov/statfacts/html/melan.html. Published 2018. Accessed May 15, 2019.

5. ACS. Key Statistics for Melanoma Skin Cancer. https://www.cancer.org/cancer/melanoma-skin-cancer/about/key-statistics.html. Accessed April 6, 2020.

6. Bhatia S, Tykodi SS, Thompson JA. Treatment of metastatic melanoma: an overview. Oncology (Williston Park). 2009;23(6):488-496. http://www.ncbi.nlm.nih.gov/pubmed/19544689\%0Ahttp://www.pubmedcentral.nih.gov/a rticlerender.fcgi?artid=PMC2737459.

7. Gadeliya Goodson A, Grossman D. Strategies for early melanoma detection: Approaches to the patient with nevi. J Am Acad Dermatol. 2009. doi:10.1016/j.jaad.2008.10.065

8. Filippi AR, Fava P, Badellino S, Astrua C, Ricardi U, Quaglino P. Radiotherapy and 
immune checkpoints inhibitors for advanced melanoma. Radiother Oncol. 2016.

doi:10.1016/j.radonc.2016.06.003

9. Song X, Zhao Z, Barber B, Farr AM, Ivanov B, Novich M. Overall survival in patients with metastatic melanoma. Curr Med Res Opin. 2015.

doi:10.1185/03007995.2015.1021904

10. Karimkhani C, Green AC, Nijsten T, et al. The global burden of melanoma: results from the Global Burden of Disease Study 2015. Br J Dermatol. 2017;177(1):134-140. doi:10.1111/bjd.15510

11. Chang CK, Jacobs IA, Vizgirda VM, Salti GI. Melanoma in the elderly patient. Arch Surg. 2003. doi:10.1001/archsurg.138.10.1135

12. Tas F, Erturk K. Patient age and cutaneous malignant melanoma: Elderly patients are likely to have more aggressive histological features and poorer survival. Mol Clin Oncol. 2017. doi:10.3892/mco.2017.1439

13. Lui P, Cashin R, Machado M, Hemels M, Corey-Lisle PK, Einarson TR. Treatments for metastatic melanoma: Synthesis of evidence from randomized trials. Cancer Treat Rev. 2007;33(8):665-680. doi:10.1016/j.ctrv.2007.06.004

14. Bhatia S, Tykodi SS, Thompson JA. Treatment of metastatic melanoma: An overview. Oncology. 2009.

15. Rao RD, Holtan SG, Ingle JN, et al. Combination of and paclitaxel and carboplatin as second-line therapy for patients with metastatic melanoma. Cancer. 2006;106(2):375-382. doi:10.1002/cncr.21611

16. Legha SS, Ring S, Bedikian A, et al. Treatment of metastatic melanoma with combined chemotherapy containing cisplatin, vinblastine and dacarbazine (CVD) and biotherapy 
using interleukin-2 and interferon-. Ann Oncol. 1996.

doi:10.1093/oxfordjournals.annonc.a010762

17. Topalian SL, Drake CG, Pardoll DM. Immune checkpoint blockade: a common denominator approach to cancer therapy. Cancer Cell. 2015;27(4):450-461. doi:10.1016/j.ccell.2015.03.001

18. Wei SC, Duffy CR, Allison JP. Fundamental mechanisms of immune checkpoint blockade therapy. Cancer Discov. 2018;8(9):1069-1086. doi:10.1158/2159-8290.CD-18-0367

19. Eggermont AMM, Chiarion-Sileni V, Grob J-J, et al. Prolonged Survival in Stage III Melanoma with Ipilimumab Adjuvant Therapy. N Engl J Med. 2016;375(19):1845-1855. doi:10.1056/NEJMoa1611299

20. Larkin J, Chiarion-Sileni V, Gonzalez R, et al. Combined Nivolumab and Ipilimumab or Monotherapy in Untreated Melanoma. N Engl J Med. 2015;373(1):23-34. doi:10.1056/NEJMoa1504030

21. Robert C, Schachter J, Long G V, et al. Pembrolizumab versus Ipilimumab in Advanced Melanoma. N Engl J Med. 2015;372(26):2521-2532. doi:10.1056/NEJMoa1503093

22. Robert C, Long G V., Schachter J, et al. Long-term outcomes in patients (pts) with ipilimumab (ipi)-naive advanced melanoma in the phase 3 KEYNOTE-006 study who completed pembrolizumab (pembro) treatment. J Clin Oncol. 2018;35(15_suppl):95049504. doi:10.1200/jco.2017.35.15_suppl.9504

23. Hodi FS, Chiarion-Sileni V, Gonzalez R, et al. Nivolumab plus ipilimumab or nivolumab alone versus ipilimumab alone in advanced melanoma (CheckMate 067): 4-year outcomes of a multicentre, randomised, phase 3 trial. Lancet Oncol. 2018;19(11):1480-1492. doi:10.1016/S1470-2045(18)30700-9 
24. Lewis MG. Melanoma. 1st ed. (Kaufman HL, Mehnert JM, eds.). Springer International Publishing; 2011. doi:10.1007/978-3-642-80725-1_9

25. Liu FX, Ou W, Diede SJ, Whitman ED. Real-world experience with pembrolizumab in patients with advanced melanoma. Medicine (Baltimore). 2019.

doi:10.1097/md.0000000000016542

26. WHO. Multimorbidity. Technical Series on Safer Primary Care. World Heal Organ. 2016. doi:10.1097/01.NURSE.0000524761.58624.1f

27. Pache B, Vollenweider P, Waeber G, Marques-Vidal P. Prevalence of measured and reported multimorbidity in a representative sample of the Swiss population Disease epidemiology - Chronic. BMC Public Health. 2015. doi:10.1186/s12889-015-1515-x

28. Sambamoorthi U, Tan X, Deb A. Multiple chronic conditions and healthcare costs among adults. Expert Rev Pharmacoeconomics Outcomes Res. 2015.

doi:10.1586/14737167.2015.1091730

29. Salive ME. Multimorbidity in older adults. Epidemiol Rev. 2013. doi:10.1093/epirev/mxs009

30. Yarnall AJ, Sayer AA, Clegg A, Rockwood K, Parker S, Hindle J V. New horizons in multimorbidity in older adults. Age Ageing. 2017. doi:10.1093/ageing/afx150

31. Buja A, Claus M, Perin L, et al. Multimorbidity patterns in high-need, high-cost elderly patients. PLoS One. 2018. doi:10.1371/journal.pone.0208875

32. Bebe FN, Hu S, Brown TL, Tulp OL. Role, extent, and impact of comorbidity on prognosis and survival in advanced metastatic melanoma: A review. J Clin Aesthet Dermatol. 2019.

33. Grann AF, Frøslev T, Olesen AB, Schmidt H, Lash TL. The impact of comorbidity and 
stage on prognosis of Danish melanoma patients, 1987-2009: A registry-based cohort study. Br J Cancer. 2013. doi:10.1038/bjc.2013.246

34. Schubert-Fritschle G, Schlesinger-Raab A, Hein R, et al. Quality of life and comorbidity in localized malignant melanoma: Results of a German population-based cohort study. Int J Dermatol. 2013. doi:10.1111/j.1365-4632.2011.05401.x

35. Peddi P, Oh JH, Kim KB, et al. Impact of comorbidities on overall survival of high-risk and advanced melanoma. ASCO Meet Abstr . 2012;30(15_suppl):8536. doi:10.1200/jco.2012.30.15_suppl.8536

36. Ma Q, Shilkrut M, Zhao Z, Li M, Batty N, Barber B. Autoimmune comorbidities in patients with metastatic melanoma: A retrospective analysis of us claims data. $B M C$ Cancer. 2018. doi:10.1186/s12885-018-4051-0

37. Niedzwiedz CL, Knifton L, Robb KA, Katikireddi SV, Smith DJ. Depression and anxiety among people living with and beyond cancer: A growing clinical and research priority. BMC Cancer. 2019. doi:10.1186/s12885-019-6181-4

38. Park EM, Gelber S, Rosenberg SM, et al. Anxiety and Depression in Young Women With Metastatic Breast Cancer: A Cross-Sectional Study. Psychosomatics. 2018. doi:10.1016/j.psym.2018.01.007

39. Peng YN, Huang ML, Kao CH. Prevalence of depression and anxiety in colorectal cancer patients: A literature review. Int J Environ Res Public Health. 2019. doi:10.3390/ijerph16030411

40. Watts S, Leydon G, Birch B, et al. Depression and anxiety in prostate cancer: A systematic review and meta-analysis of prevalence rates. BMJ Open. 2014. doi:10.1136/bmjopen-2013-003901 
41. Baillargeon J, Kuo YF, Lin YL, Raji MA, Singh A, Goodwin JS. Effect of mental disorders on diagnosis, treatment, and survival of older adults with colon cancer. $J$ Am Geriatr Soc. 2011. doi:10.1111/j.1532-5415.2011.03481.x

42. Danlos FX, Voisin AL, Dyevre V, et al. Safety and efficacy of anti-programmed death 1 antibodies in patients with cancer and pre-existing autoimmune or inflammatory disease. Eur J Cancer. 2018. doi:10.1016/j.ejca.2017.12.008

43. Menzies AM, Johnson DB, Ramanujam S, et al. Anti-PD-1 therapy in patients with advanced melanoma and preexisting autoimmune disorders or major toxicity with ipilimumab. Ann Oncol. 2017. doi:10.1093/annonc/mdw443

44. Snyder CF, Frick KD, Herbert RJ, et al. Comorbid condition care quality in cancer survivors: role of primary care and specialty providers and care coordination. J Cancer Surviv. 2015. doi:10.1007/s11764-015-0440-4

45. Garg R, Sambamoorthi U, Tan X, Basu SK, Haggerty T, Kelly KM. Impact of diffuse large B-cell lymphoma on visits to different provider specialties among elderly Medicare beneficiaries: Challenges for care coordination. Transl Behav Med. 2018. doi:10.1093/tbm/ibx071

46. Nipp R, Tramontano AC, Kong CY, et al. Disparities in cancer outcomes across age, sex, and race/ethnicity among patients with pancreatic cancer. Cancer Med. 2018. doi:10.1002/cam4.1277

47. Datta GD, Neville BA, Kawachi I, Datta NS, Earle CC. Marital status and survival following bladder cancer. J Epidemiol Community Health. 2009. doi:10.1136/jech.2008.082438

48. Krimphove MJ, Tully KH, Friedlander DF, et al. Adoption of immunotherapy in the 
community for patients diagnosed with metastatic melanoma. J Immunother Cancer. 2019. doi:10.1186/s40425-019-0782-y

49. Shih YCT, Elting LS, Halpern MT. Factors associated with immunotherapy use among newly diagnosed cancer patients. Med Care. 2009. doi:10.1097/MLR.0b013e31819a5b2b

50. Guy GP, Ekwueme DU, Yabroff KR, et al. Economic burden of cancer survivorship among adults in the United States. J Clin Oncol. 2013. doi:10.1200/JCO.2013.49.1241

51. De Oliveira C, Pataky R, Bremner KE, et al. Estimating the cost of cancer care in British Columbia and Ontario: A Canadian inter-provincial comparison. Healthc Policy. 2017. doi:10.12927/hcpol.2017.25024

52. Yabroff KR, Lamont EB, Mariotto A, et al. Cost of care for elderly cancer patients in the United States. J Natl Cancer Inst. 2008. doi:10.1093/jnci/djn103

53. Tsao H, Rogers GS, Sober AJ. An estimate of the annual direct cost of treating cutaneous melanoma. J Am Acad Dermatol. 1998;38(5 I):669-680. doi:10.1016/S01909622(98)70195-1

54. Guy GP, Ekwueme DU, Tangka FK, Richardson LC. Melanoma treatment costs: A systematic review of the literature, 1990-2011. Am J Prev Med. 2012. doi:10.1016/j.amepre.2012.07.031

55. Seidler AM, Pennie ML, Veledar E, Culler SD, Chen SC. Economic Burden of Melanoma in the Elderly Population. Arch Dermatol. 2010. doi:10.1001/archdermatol.2009.389

56. Dranitsaris G, Zhu X, Adunlin G, Vincent MD. Cost effectiveness vs. affordability in the age of immuno-oncology cancer drugs. Expert Rev Pharmacoeconomics Outcomes Res. 2018. doi:10.1080/14737167.2018.1467270

57. Andrews A. Treating with Checkpoint Inhibitors-Figure \$1 Million per Patient. Am Heal 
drug benefits. 2015.

58. Ghate SR, Ionescu-Ittu R, Burne R, et al. Healthcare resource utilization in patients with metastatic melanoma receiving first-line therapy with dabrafenib + trametinib versus nivolumab or pembrolizumab monotherapy. Curr Med Res Opin. 2018. doi:10.1080/03007995.2018.1501351

59. Zulman DM, Chee CP, Wagner TH, et al. Multimorbidity and healthcare utilisation among high-cost patients in the US Veterans Affairs Health Care System. BMJ Open. 2015. doi:10.1136/bmjopen-2015-007771

60. Wilkinson R, Marmot M. Determinants of Health. The Solid Facts. 2nd Edition. World Heal Organiztaion. 2003. doi:10.1016/j.jana.2012.03.001

61. Phillips KA, Morrison KR, Andersen R, Aday LA. Understanding the context of healthcare utilization: assessing environmental and provider-related variables in the behavioral model of utilization. Health Serv Res. 1998.

62. Siegel RL, Miller KD, Jemal A. Cancer statistics, 2019. CA Cancer J Clin. 2019;69(1):734. doi:10.3322/caac. 21551

63. Glazer AM, Winkelmann RR, Farberg AS, Rigel DS. Analysis of trends in US melanoma incidence and mortality. JAMA Dermatology. 2017. doi:10.1001/jamadermatol.2016.4512

64. Sarfati D, Koczwara B, Jackson C. The impact of comorbidity on cancer and its treatment. CA Cancer J Clin. 2016;66(4):337-350. doi:10.3322/caac.21342

65. Kenzik K, Richman J, Kent EE, Pisu M, Bhatia S. Impact of precancer multimorbidity clusters on survival and functional outcomes after cancer in older patients. J Clin Oncol. 2016. doi:10.1200/jco.2016.34.7_suppl.291

66. Meneses K, Benz R, Azuero A, Jablonski-Jaudon R, McNees P. Multimorbidity and 
breast cancer. Semin Oncol Nurs. 2015. doi:10.1016/j.soncn.2015.02.004

67. Georgieva M, Lund J, Nielsen M, et al. The Impact of Functional Status and MultiMorbidity On Surveillance Use Among Medicare Beneficiaries with Bladder Cancer. Value Heal. 2018. doi:10.1016/j.jval.2018.04.951

68. LUQUE-FERNANDEZ MA, Redondo-Sanchez D, RODRIGUEZ-BARRANCO M, Garcia MCC, Marcos-Gragera R, SANCHEZ-PEREZ MJ. The pattern of Comorbidities and Associated Risk Factors among Colorectal Cancer Patients in Spain: CoMCoR study. bioRxiv. 2019. doi:10.1101/526673

69. Garbe C, Eigentler TK, Keilholz U, Hauschild A, Kirkwood JM. Systematic Review of Medical Treatment in Melanoma: Current Status and Future Prospects. Oncologist. 2011. doi:10.1634/theoncologist.2010-0190

70. Shrestha A, Martin C, Burton M, Walters S, Collins K, Wyld L. Quality of life versus length of life considerations in cancer patients: A systematic literature review. Psychooncology. 2019. doi:10.1002/pon.5054

71. Gutzmer R, Koop A, Meier F, et al. Programmed cell death protein-1 (PD-1) inhibitor therapy in patients with advanced melanoma and preexisting autoimmunity or ipilimumab-triggered autoimmunity. Eur J Cancer. 2017. doi:10.1016/j.ejca.2016.12.038

72. Donia M, Kimper-Karl ML, Høyer KL, Bastholt L, Schmidt H, Svane IM. The majority of patients with metastatic melanoma are not represented in pivotal phase III immunotherapy trials. Eur J Cancer. 2017. doi:10.1016/j.ejca.2016.12.017

73. Goodman RA, Posner SF, Huang ES, Parekh AK, Koh HK. Defining and measuring chronic conditions: Imperatives for research, policy, program, and practice. Prev Chronic Dis. 2013. doi: $10.5888 /$ pcd10.120239 
74. AARDA. Autoimmune Disease List. https://www.aarda.org/diseaselist/. Published 2019.

75. Halter JB, Musi N, Horne FMF, et al. Diabetes and cardiovascular disease in older adults: Current status and future directions. Diabetes. 2014. doi:10.2337/db14-0020

76. Williams A, Kamper SJ, Wiggers JH, et al. Musculoskeletal conditions may increase the risk of chronic disease: A systematic review and meta-analysis of cohort studies. BMC Med. 2018. doi:10.1186/s12916-018-1151-2

77. Koene RJ, Prizment AE, Blaes A, Konety SH. Shared risk factors in cardiovascular disease and cancer. Circulation. 2016. doi:10.1161/CIRCULATIONAHA.115.020406

78. Vincent L, Leedy D, Masri SC, Cheng RK. Cardiovascular Disease and Cancer: Is There Increasing Overlap? Curr Oncol Rep. 2019. doi:10.1007/s11912-019-0796-0

79. Giovannucci E, Harlan DM, Archer MC, et al. Diabetes and cancer: A consensus report. In: Diabetes Care. ; 2010. doi:10.2337/dc10-0666

80. Ferrucci L, Giallauria F, Guralnik JM. Epidemiology of Aging. Radiol Clin North Am. 2008. doi:10.1016/j.rcl.2008.07.005

81. Gurney J, Sarfati D, Stanley J. The impact of patient comorbidity on cancer stage at diagnosis. Br J Cancer. 2015;113(9):1375-1380. doi:10.1038/bjc.2015.355

82. Fairweather D, Frisancho-Kiss S, Rose NR. Sex differences in autoimmune disease from a pathological perspective. Am J Pathol. 2008. doi:10.2353/ajpath.2008.071008

83. Carpenter DM, Thorpe CT, Alexander DS, et al. The Relationship Between Social Support, Social Constraint, and Psychological Adjustment for Patients with Rare Autoimmune Disease. Curr Rheumatol Rev. 2016. doi:10.2174/1573397112666160719163145

84. Kasparian NA. Psychological stress and melanoma: Are we meeting our patients' 
psychological needs? Clin Dermatol. 2013. doi:10.1016/j.clindermatol.2011.11.005

85. Kasparian NA, McLoone JK, Butow PN. Psychological responses and coping strategies among patients with malignant melanoma: A systematic review of the literature. Arch Dermatol. 2009. doi:10.1001/archdermatol.2009.308

86. Kasparian NA, Meiser B, Butow PN, Simpson JM, Mann GJ. Predictors of psychological distress among individuals with a strong family history of malignant melanoma. Clin Genet. 2008. doi:10.1111/j.1399-0004.2007.00949.x

87. Anuk D, Özkan M, Kizir A, Özkan S. The characteristics and risk factors for common psychiatric disorders in patients with cancer seeking help for mental health. $B M C$ Psychiatry. 2019. doi:10.1186/s12888-019-2251-z

88. Pitman A, Suleman S, Hyde N, Hodgkiss A. Depression and anxiety in patients with cancer. BMJ. 2018. doi:10.1136/bmj.k1415

89. Hung C-T, Chen Y-J, Chan J-C, et al. Psychological distress, social support, selfmanagement ability and utilization of social resources for female patients with cancer in Oncology Outpatient Settings in Taiwan. Support Care Cancer. 2019;[Epub ahea. doi:10.1007/s00520-019-05143-y

90. Amini A, Rusthoven CG, Waxweiler T V., et al. Association of health insurance with outcomes in adults ages 18 to 64 years with melanoma in the United States. J Am Acad Dermatol. 2016. doi:10.1016/j.jaad.2015.09.054

91. Read WL, Tierney RM, Page NC, et al. Differential prognostic impact of comorbidity. $J$ Clin Oncol. 2004. doi:10.1200/JCO.2004.08.040

92. Kaufman HL, Margolin K, Sullivan R. Management of Metastatic Melanoma in 2018. JAMA Oncol. 2018;4(6):857. doi:10.1001/jamaoncol.2018.0170 
93. Eggermont AMM, Chiarion-Sileni V, Grob JJ, et al. Adjuvant ipilimumab versus placebo after complete resection of high-risk stage III melanoma (EORTC 18071): A randomised, double-blind, phase 3 trial. Lancet Oncol. 2015;16(5):522-530. doi:10.1016/S14702045(15)70122-1

94. Bennette CS, Torres AZ, Tucker M, et al. Diffusion of innovation in oncology: A case study of immuno-oncology (IO) adoption for advanced non-small lung cancer (aNSCLC) patients across practices in the US. J Clin Oncol. 2018;36(15_suppl):6537-6537. doi:10.1200/JCO.2018.36.15_suppl.6537

95. Cybulska-Stopa B, Ługowska I, Jagodzińska-Mucha P, et al. Immune checkpoint inhibitors therapy in older patients ( $\geq 70$ years) with metastatic melanoma: a multicentre study. Postep Dermatol Alergol. 2019;36(5):566-571. doi:10.5114/ada.2018.79940

96. Ben-Betzalel G, Steinberg-Silman Y, Stoff R, et al. Immunotherapy comes of age in octagenarian and nonagenarian metastatic melanoma patients. Eur J Cancer. 2019. doi:10.1016/j.ejca.2018.12.012

97. van Holstein Y, Kapiteijn E, Bastiaannet E, van den Bos F, Portielje J, de Glas NA. Efficacy and Adverse Events of Immunotherapy with Checkpoint Inhibitors in Older Patients with Cancer. Drugs Aging. 2019;36(10):927-938. doi:10.1007/s40266-019$00697-2$

98. Hegde UP, Parmar H V, Stevenson CE. Clinical heterogeneity of elderly melanoma patients in the real-world setting: Potential implications for treatment outcomes following immune based therapy. In: Journal of Clinical Oncology. Vol 37. ; 2019:e21026-e21026. doi:0.1200/JCO.2019.37.15_suppl.e21026

99. Middleton MR, Grob JJ, Aaronson N, et al. Randomized phase III study of temozolomide 
versus dacarbazine in the treatment of patients with advanced metastatic malignant melanoma. J Clin Oncol. 2000. doi:10.1200/jco.2000.18.1.158

100. Sandru A, Voinea S, Panaitescu E, Blidaru A. Survival rates of patients with metastatic malignant melanoma. J Med Life. 2014.

101. Walker MS, Reyes C, Kerr J, Satram-Hoang S, Stepanski EJ. Treatment patterns and outcomes among patients with metastatic melanoma treated in community practice. Int $J$ Dermatol. 2014. doi:10.1111/ijd.12427

102. Whitman ED, Liu FX, Cao X, Diede SJ, Haiderali A, Abernethy AP. Treatment patterns and outcomes for patients with advanced melanoma in US oncology clinical practices. Futur Oncol. 2019;15(5):459-471. doi:10.2217/fon-2018-0620

103. Cowey CL, Liu FX, Boyd M, Aguilar KM, Krepler C. Real-world treatment patterns and clinical outcomes among patients with advanced melanoma. Medicine (Baltimore). 2019. doi:10.1097/md.0000000000016328

104. Pavlick A, Weber J. Managing Checkpoint Inhibitor Symptoms and Toxicity for Metastatic Melanoma. In: Cutaneous Melanoma. ; 2020. doi:10.1007/978-3-030-050702_60

105. Orloff M. Melanoma Immunotherapy in the Elderly. Curr Oncol Rep. 2018. doi:10.1007/s11912-018-0656-3

106. Unger JM, Hershman DL, Fleury ME, Vaidya R. Association of Patient Comorbid Conditions with Cancer Clinical Trial Participation. JAMA Oncol. 2019. doi:10.1001/jamaoncol.2018.5953

107. Kent E, Mollica M, Klabunde CN, et al. Examining the relative influence of multimorbidity on variations in older cancer patients' experiences with care. J Clin Oncol. 
2018. doi:10.1200/jco.2018.36.30_suppl.14

108. Garg R, Shen C, Sambamoorthi N, Kelly K, Sambamoorthi U. Type of Multimorbidity and Patient-Doctor Communication and Trust among Elderly Medicare Beneficiaries. Int J Family Med. 2016. doi:10.1155/2016/8747891

109. Given B, Given CW. Older adults and cancer treatment. In: Cancer. ; 2008. doi:10.1002/cncr.23939

110. Shahrokni A, Wu AJ, Carter J, Lichtman SM. Long-term Toxicity of Cancer Treatment in Older Patients. Clin Geriatr Med. 2016. doi:10.1016/j.cger.2015.08.005

111. Lai H, Lai S, Krongrad A, Trapido E, Page J, McCoy C. The effect of marital status on survival in late-stage cancer patients: an analysis based on surveillance, epidemiology, and end results (SEER) data, in the United States. Int J Behav Med. 1999;6(2):150-176. doi:10.1207/s15327558ijbm0602_4

112. McLaughlin JM, Fisher JL, Paskett ED. Marital status and stage at diagnosis of cutaneous melanoma: results from the Surveillance Epidemiology and End Results (SEER) program, 1973-2006. Cancer. 2011;117(9):1984-1993. doi:10.1002/cncr.25726

113. Walker JL. The Diffusion of Innovations among the American States. Am Polit Sci Rev. 1969. doi: $10.2307 / 1954434$

114. Carter LE, LaPlant JT. Diffusion of Health Care Policy Innovation in the United States. State Local Gov Rev. 1997. doi:10.1177/0160323x9702900102

115. Agha L, Molitor D. The Local Influence of Pioneer Investigators on Technology Adoption: Evidence from New Cancer Drugs. Rev Econ Stat. 2018. doi:10.1162/rest_a_00670

116. Koroukian SM, Bakaki PM, Owusu C, Earle CC, Cooper GS. Cancer outcomes in low- 
income elders: is there an advantage to being on Medicaid? Medicare Medicaid Res Rev. 2012. doi:10.5600/mmrr.002.02.a06

117. Lisa MH, Louder A, Winfree K, Zhu YE, Oton AB, Nair R. Factors associated with adherence to and treatment duration of erlotinib among patients with non-small cell lung cancer. J Manag Care Spec Pharm. 2017. doi:10.18553/jmcp.2017.16389

118. Al-Qurayshi Z, Crowther JE, Hamner JB, Ducoin C, Killackey MT, Kandil E. Disparities of immunotherapy utilization in patients with stage III cutaneous melanoma: A national perspective. Anticancer Res. 2018. doi:10.21873/anticanres.12536

119. Haque W, Verma V, Butler EB, Teh BS. Racial and Socioeconomic Disparities in the Delivery of Immunotherapy for Metastatic Melanoma in the United States. J Immunother. 2019. doi:10.1097/CJI.0000000000000264

120. Verma V, Haque W, Cushman TR, et al. Racial and Insurance-related Disparities in Delivery of Immunotherapy-type Compounds in the United States. J Immunother. 2019. doi:10.1097/CJI.0000000000000253

121. Keating NL, O’Malley AJ, Onnela JP, Gray SW, Landon BE. Association of Physician Peer Influence With Subsequent Physician Adoption and Use of Bevacizumab. JAMA Netw open. 2020. doi:10.1001/jamanetworkopen.2019.18586

122. Ma X, Yu H. Global burden of cancer. Yale J Biol Med. 2006. doi:10.1016/s01406736(97)90017-9

123. Fitzmaurice C, Dicker D, Pain A, et al. The Global Burden of Cancer 2013. JAMA Oncol. 2015. doi:10.1001/jamaoncol.2015.0735

124. Yabroff KR, Lund J, Kepka D, Mariotto A. Economic burden of cancer in the United States: Estimates, projections, and future research. Cancer Epidemiol Biomarkers Prev. 
2011. doi:10.1158/1055-9965.EPI-11-0650

125. Davidoff AJ, Erten M, Shaffer T, et al. Out-of-pocket health care expenditure burden for Medicare beneficiaries with cancer. Cancer. 2013. doi:10.1002/cncr.27848

126. Narang AK, Nicholas LH. Out-of-pocket spending and financial burden among medicare beneficiaries with cancer. JAMA Oncol. 2017. doi:10.1001/jamaoncol.2016.4865

127. Park J, Look KA. Health Care Expenditure Burden of Cancer Care in the United States. Inq (United States). 2019. doi:10.1177/0046958019880696

128. Chang CL, Schabert VF, Munakata J, et al. Comparative healthcare costs in patients with metastatic melanoma in the USA. Melanoma Res. 2015.

doi:10.1097/CMR.0000000000000159

129. Oh A, Tran DM, Mc LCD, et al. Cost-effectiveness of nivolumab-ipilimumab combination therapy compared with monotherapy for first-line treatment of metastatic melanoma in the United States. J Manag Care Spec Pharm. 2017. doi:10.18553/jmcp.2017.23.6.653

130. Toy EL, Vekeman F, Lewis MC, Oglesby AK, Duh MS. Costs, resource utilization, and treatment patterns for patients with metastatic melanoma in a commercially insured setting. Curr Med Res Opin. 2015. doi:10.1185/03007995.2015.1062356

131. Coughlin SS, Caplan L, Young L. A review of cancer outcomes among persons dually enrolled in Medicare and Medicaid. J Hosp Manag Heal Policy. 2018. doi:10.21037/jhmhp.2018.07.04

132. Shih YCT, Smieliauskas F, Geynisman DM, Kelly RJ, Smith TJ. Trends in the cost and use of targeted cancer therapies for the privately insured nonelderly: 2001 To 2011. J Clin Oncol. 2015. doi:10.1200/JCO.2014.58.2320 
133. Jain V, Venigalla S, Reddy VK, Lukens JN, Mitchell TC, Shabason JE. Association of Insurance Status with Presentation, Treatment, and Survival in Melanoma in the Era of Immune Checkpoint Inhibitors. J Immunother. 2020. doi:10.1097/CJI.0000000000000294

134. Lohr KN. Medicare: a strategy for quality assurance. J Qual Assur. 1991. doi:10.1111/j.1945-1474.1991.tb00115.x

135. Hegde UP, Grant-Kels JM. Metastatic melanoma in the older patient: Special considerations. Clin Dermatol. 2013. doi:10.1016/j.clindermatol.2012.08.011

136. Kline RM, Bazell C, Smith E, Schumacher H, Rajkumar R, Conway PH. Centers for Medicare and Medicaid Services: Using an Episode-Based Payment Model to Improve Oncology Care. J Oncol Pract. 2015. doi:10.1200/jop.2014.002337

137. King DE, Xiang J, Pilkerton CS. Multimorbidity trends in United States adults, 19882014. J Am Board Fam Med. 2018. doi:10.3122/jabfm.2018.04.180008

138. Lochner KA, Cox CS. Prevalence of multiple chronic conditions among medicare beneficiaries, United States, 2010. Prev Chronic Dis. 2013. doi:10.5888/pcd10.120137

139. Schneider KM, O'Donnell BE, Dean D. Prevalence of multiple chronic conditions in the United States' Medicare population. Health Qual Life Outcomes. 2009. doi:10.1186/1477$7525-7-82$

140. Wang L, Si L, Cocker F, Palmer AJ, Sanderson K. A Systematic Review of Cost-ofIllness Studies of Multimorbidity. Appl Health Econ Health Policy. 2018. doi:10.1007/s40258-017-0346-6

141. Rim SH, Guy GP, Yabroff KR, McGraw KA, Ekwueme DU. The impact of chronic conditions on the economic burden of cancer survivorship: a systematic review. Expert Rev Pharmacoeconomics Outcomes Res. 2016. doi:10.1080/14737167.2016.1239533 
142. Picco L, Achilla E, Abdin E, et al. Economic burden of multimorbidity among older adults: Impact on healthcare and societal costs. BMC Health Serv Res. 2016. doi:10.1186/s12913-016-1421-7

143. Rai P, Shen C, Kolodney J, Kelly K, Scott V, Sambamoorthi U. Prevalence and Risk Factors for Multimorbidity in Elderly US Patients with Late-Stage Melanoma.; 2020.

144. Chopra I, Mattes MD, Findley P, Tan X, Dwibedi N, Sambamoorthi U. Impact of incident cancer on short-term coronary artery disease-related healthcare expenditures among Medicare beneficiaries. JNCCN J Natl Compr Cancer Netw. 2019.

doi:10.6004/jnccn.2018.7078

145. CPI. Bureau of Labor Statistics website. https://www.bls.gov/cpi/. Accessed February 24, 2020.

146. Manning WG, Mullahy J. Estimating log models: To transform or not to transform? $J$ Health Econ. 2001. doi:10.1016/S0167-6296(01)00086-8

147. Tangka FK, Trogdon JG, Richardson LC, Howard D, Sabatino SA, Finkelstein EA. Cancer treatment cost in the United States: Has the burden shifted over time? Cancer. 2010. doi:10.1002/cncr. 25150

148. Dudley N, Lee SJ, Stijacic-Cenzer I, Ritchie CS. Prevalence of Multimorbidity Among Older Adults With Advanced Illness Visits to U.S. Subspecialty Clinics. J Pain Symptom Manage. 2018. doi:10.1016/j.jpainsymman.2018.03.012

149. Hamid O, Robert C, Daud A, et al. Five-year survival outcomes for patients with advanced melanoma treated with pembrolizumab in KEYNOTE-001. Ann Oncol. 2019. doi:10.1093/annonc/mdz011

150. Hodi FS, O’Day SJ, McDermott DF, et al. Improved survival with ipilimumab in patients 
with metastatic melanoma. $N$ Engl J Med. 2010. doi:10.1056/NEJMoa1003466 


\section{APPENDIX}

Appendix 7.1: List of autoimmune diseases

\begin{tabular}{|c|c|}
\hline S.No. & Autoimmune disease \\
\hline 1 & Achalasia \\
\hline 2 & Addison's disease \\
\hline 3 & Adult Still's disease \\
\hline 4 & Agammaglobulinemia \\
\hline 5 & Alopecia areata \\
\hline 6 & Amyloidosis \\
\hline 7 & Ankylosing spondylitis \\
\hline 8 & Anti-GBM/Anti-TBM nephritis \\
\hline 9 & Antiphospholipid syndrome \\
\hline 10 & Autoimmune angioedema \\
\hline 11 & Autoimmune dysautonomia \\
\hline 12 & Autoimmune encephalomyelitis \\
\hline 13 & Autoimmune heptatitis \\
\hline 14 & Autoimmune inner ear disease (AIED) \\
\hline 15 & Autoimmune myocarditis \\
\hline 16 & Autoimmune oophoritis \\
\hline 17 & Autoimmune orchitis \\
\hline 18 & Autoimmune pancreatitis \\
\hline 19 & Autoimmune retinopathy \\
\hline 20 & Autoimmune urticaria \\
\hline 21 & Axonal \& neuronal neuropathy (AMAN) \\
\hline 22 & Balo disease \\
\hline 23 & Benign mucosal pemphigoid \\
\hline 24 & Castleman disease \\
\hline 25 & Celiac disease \\
\hline 27 & Chagas disease \\
\hline 27 & Chronic inflammatory demyelinating polyneuropathy (CIDP) \\
\hline 28 & Chronic recurrent multifocal osteomyelitis (CRMO) \\
\hline 29 & Cicatricial pemphigoid \\
\hline 30 & Cogan's syndrome \\
\hline 31 & Cold agglutinin disease \\
\hline 32 & Congenital heart block \\
\hline 33 & Coxsackie myocarditis \\
\hline 34 & CREST syndrome \\
\hline 35 & Crohn's disease \\
\hline 36 & Dermatitis herpetiformis \\
\hline 37 & Dermatomyositis \\
\hline 38 & Devic's disease (neuromyelitis optica) \\
\hline
\end{tabular}




\begin{tabular}{|c|c|}
\hline S.No. & Autoimmune disease \\
\hline 39 & Discoid lupus \\
\hline 40 & Dressler's syndrome \\
\hline 41 & Endometriosis \\
\hline 42 & Eosinophilic esophagitis (EoE) \\
\hline 43 & Eosinophilic fascitis \\
\hline 44 & Erythema nodosum \\
\hline 45 & Evan syndrome \\
\hline 46 & Fibromyalgia \\
\hline 47 & Fibrosing alveolitis \\
\hline 48 & Giant cell myocarditis \\
\hline 49 & Glomerulonephritis \\
\hline 50 & Goodpasture's syndrome \\
\hline 51 & Grave's disease \\
\hline 52 & Guillain-Barre syndrome \\
\hline 53 & Hashimoto's thyroiditis \\
\hline 54 & Hemolytic anemia \\
\hline 55 & Herpes gestationis or pemphigoid gestationis (PG) \\
\hline 56 & Hiradenitis Suppurativa (HS) (Acne inversa) \\
\hline 57 & Hypogammalglobulinemia \\
\hline 58 & IgA Nephropathy \\
\hline 59 & IgG4-related sclerosing disease \\
\hline 60 & Immune thrombocytopic purpura (ITP) \\
\hline 61 & Inclusion body myositis (IBM) \\
\hline 62 & Interstitial cystitis \\
\hline 63 & Kawasaki disease \\
\hline 64 & Lambert-eaton syndrome \\
\hline 65 & Leukocytoclastic vasculitis \\
\hline 66 & Lichen planus \\
\hline 67 & Ligenous conjuctivitis \\
\hline 68 & Linear IgA disease (LAD) \\
\hline 69 & Lupus \\
\hline 70 & Lyme disease chronic \\
\hline 71 & Meniere's disease \\
\hline 72 & Mixed connective tissure disease \\
\hline 73 & Mooren's ulcer \\
\hline 74 & Mucha-Habermann disease \\
\hline 75 & Multifocal motor neuropathy \\
\hline 76 & Multiple sclerosis \\
\hline 77 & Myasthenia gravis \\
\hline 78 & Myositis \\
\hline
\end{tabular}




\begin{tabular}{|c|c|}
\hline S.No. & Autoimmune disease \\
\hline 79 & Narcolepsy \\
\hline 80 & Neuromyelitis optica \\
\hline 81 & Neutropenia \\
\hline 82 & Ocular cicatricial pemphigoid \\
\hline 83 & Optic neuritis \\
\hline 84 & Palindromic rheumatism \\
\hline 85 & Paraneoplastic cerebellar degeneration \\
\hline 86 & Paroxysmal noctural hemoglobinuria \\
\hline 87 & Parry Romberg syndrome \\
\hline 88 & Pars planitis (peripheral uveitis) \\
\hline 89 & Parsonage-Turner syndrome \\
\hline 90 & Pemphigus \\
\hline 91 & Peripheral neuropathy \\
\hline 92 & Perivenous encephalomyelitis \\
\hline 93 & Pernicious anemia \\
\hline 94 & $\begin{array}{l}\text { Polyneuropathy, organomegaly, endocrinopathy, monoclonal } \\
\text { gammopathy, skin changes (POEMS syndrome) }\end{array}$ \\
\hline 95 & Polyglandular syndromes type I, II, III \\
\hline 96 & Polymyositis \\
\hline 97 & Postmyocardial infarction syndrome \\
\hline 98 & Postpericardiotomy syndrome \\
\hline 99 & Primary biliary cirrhosis \\
\hline 100 & Primary sclerosing cholangitis \\
\hline 101 & Progesterone dermatitis \\
\hline 102 & Psoriasis \\
\hline 103 & Psoriatic arthritis \\
\hline 104 & Pure red cell aplasia \\
\hline 105 & Pyoderma gangrenosum \\
\hline 106 & Raynaud's phenomenon \\
\hline 107 & Reactive arthritis \\
\hline 108 & Reflex sympathetic dystrophy \\
\hline 109 & Relapsing polychondritis \\
\hline 110 & Restless legs syndrome \\
\hline 111 & Retroperitoneal fibrosis \\
\hline 112 & Rheumatic fever \\
\hline 113 & Rheumatic arthritis \\
\hline 114 & Sarcoidosis \\
\hline 115 & Schmidt syndrome \\
\hline 116 & Scleritis \\
\hline 117 & Sjogren's syndrome \\
\hline 118 & Stiff person syndrome \\
\hline
\end{tabular}




\begin{tabular}{|l|l|}
\hline S.No. & Autoimmune disease \\
\hline 119 & Subacute bacterial endocarditis \\
\hline 120 & Susac's syndrome \\
\hline 121 & Sympathetic ophthalmia \\
\hline 122 & Takayasu's arteritis \\
\hline 123 & Thrombocytopenic purpura \\
\hline 124 & Tolosa-Hunt syndrome \\
\hline 125 & Transverse myelitis \\
\hline 126 & Ulcerative colitis \\
\hline 127 & Undifferentiated connective tissue disease \\
\hline 128 & Uveitis, Panuveitis \\
\hline 129 & Vasculitis \\
\hline 130 & Vitiligo \\
\hline 131 & Vogt-Koyanagi-Harada disease \\
\hline
\end{tabular}


Appendix 7.2: Codes for identification of chemotherapy and radiation for the treatment of late-stage melanoma

\begin{tabular}{|c|c|c|c|}
\hline & Chemotherapy & HCPCS Codes & CPT Codes \\
\hline \multirow{25}{*}{ factor } & Amifostine & 'J0207' & '96374' \\
\hline & Bacillus Calmette-Guérin & '90585', '90586', 'J9031' & '90471', '90472' \\
\hline & Bleomycin & 'J9040' & '96401', '96409' \\
\hline & Carboplatin & 'J9045' & '96409', '96413'*, '96415'* \\
\hline & Cisplatin & 'J9060' & '96409', '96413',** '96415'* \\
\hline & Carmustine & 'J9050' & '96413', '96415'* \\
\hline & Dacarbazine & 'J9130' & '96409', ' 96413'* \\
\hline & Docetaxel & 'J9171 ' & '96413*' \\
\hline & Granulocyte-macrophage colony-stimulating & 'J2820' & '96365', '96366', '96372' \\
\hline & IL-2- aldesleukin & 'J9015' & '96409' \\
\hline & Interferon alfa- $2 b$ & 'C9399',* 'J9999'* & '96372', '96401' \\
\hline & Lomustine & 'J8999'*, 'S0178' & - \\
\hline & Megestrol acetate & 'J8999'*, 'S0179' & - \\
\hline & Melphalan & 'J9245' & '96409', '96413'** \\
\hline & Nab-paclitaxel & 'J9264' & - \\
\hline & Paclitaxel & 'J9265' & '96413'*, '96415'* \\
\hline & Temozolomide & 'J8700' & - \\
\hline & Vincristine & 'J9370' & '96409' \\
\hline & Vinblastine & 'J9360' & '96409' \\
\hline & Radiation & HCPCS Codes & CPT Codes \\
\hline & Intensity Modulated Radiation Therapy & - & '77301', '77338' \\
\hline & Stereotactic Body Radiation Therapy & 'G0339', 'G0340' & '77373', '77435' \\
\hline & 2D and 3D Conformal & $\begin{array}{l}\text { 'G6003', 'G6004', 'G6005', 'G6006', 'G6007', } \\
\text { 'G6008', 'G6009', 'G6010', 'G6011', 'G6012', 'G6013', } \\
\text { 'G6014' }\end{array}$ & $\begin{array}{r}\text { '77280', '77285', '77290', } \\
\text { '77295', '77402', '77407', '77412' }\end{array}$ \\
\hline & Stereotactic Radiosurgery & 'G0339', 'G0340' & '77371', '77372', '77432' \\
\hline & Brachytherapy & & $\begin{array}{l}\text { '67218', '77316', '77317', } \\
\text { '77318', '77778' }\end{array}$ \\
\hline
\end{tabular}

* Unclassified drugs/biologics; Not otherwise classified, antineoplastics; * Not included for identification of chemotherap 


\section{Appendix 7.3}

Percent with Immune Checkpoint Inhibitor (ICI) use by Selected Patient-level Characteristics among Older Adults (age > 65 years) with Incident Late-stage Melanoma during 2012 and 2015

Linked Surveillance, Epidemiology, and End Results and Medicare Claims Database

\begin{tabular}{|c|c|c|c|c|c|}
\hline Characteristics & $\begin{array}{c}\text { ALL } \\
\% \\
\end{array}$ & $\begin{array}{c}\text { ICI } \\
\% \\
\end{array}$ & $\begin{array}{c}\text { No ICI } \\
\% \\
\end{array}$ & p-value & Significance \\
\hline ALL & 100 & 5.6 & 94.4 & & \\
\hline \multicolumn{6}{|l|}{ Year of diagnosis } \\
\hline 2012 & 24.2 & 3.2 & 96.8 & 0.0008 & $* * *$ \\
\hline 2013 & 24.1 & 5.8 & 94.2 & & \\
\hline 2014 & 25.8 & 6.4 & 93.6 & & \\
\hline 2015 & 25.9 & 6.8 & 93.2 & & \\
\hline \multicolumn{6}{|l|}{ Multimorbidity } \\
\hline Yes & 84.8 & 5.5 & 94.5 & 0.62 & \\
\hline No & 15.2 & 6.0 & 94.0 & & \\
\hline \multicolumn{6}{|l|}{ Age (in years) } \\
\hline 65 to 69 & 20.2 & 6.8 & 93.2 & 0.0004 & $* * *$ \\
\hline 70 to 74 & 22.2 & 7.6 & 92.4 & & \\
\hline 75 to 79 & 20 & 5.0 & 95.0 & & \\
\hline 80 and older & 37.5 & 4.1 & 95.9 & & \\
\hline \multicolumn{6}{|l|}{ Sex } \\
\hline Females & 35.8 & 4.5 & 95.5 & 0.014 & $*$ \\
\hline Males & 64.2 & 6.2 & 93.8 & & \\
\hline \multicolumn{6}{|l|}{ Race } \\
\hline Whites & 96.1 & 5.5 & 94.5 & 0.29 & \\
\hline Non-Whites & 3.9 & 7.3 & 92.7 & & \\
\hline \multicolumn{6}{|l|}{ Marital status } \\
\hline Unmarried & 50.9 & 7.3 & 92.7 & $<0.0001$ & $* * *$ \\
\hline Married & 49.1 & 3.9 & 96.1 & & \\
\hline \multicolumn{6}{|c|}{ Dual Medicare/Medicaid eligibility } \\
\hline Yes & 3.8 & 10.0 & 90.0 & 0.0104 & $*$ \\
\hline No & 96.2 & 5.4 & 94.6 & & \\
\hline \multicolumn{6}{|l|}{ Oncologist visits } \\
\hline Yes & 20.0 & 12.5 & 87.5 & $<0.0001$ & $* * *$ \\
\hline No & 80.0 & 3.8 & 96.2 & & \\
\hline \multicolumn{6}{|l|}{ Primary care physicians } \\
\hline Yes & 62.0 & 6.2 & 93.8 & $<0.0001$ & $* * *$ \\
\hline No & 38.0 & 4.6 & 95.4 & & \\
\hline
\end{tabular}

Note: Based on 4,519 older adults with incident late-stage (Stage III/IV) melanoma continuously enrolled in Medicare Part A \& B fee-for service programs 12 months prior to incident cancer diagnosis. 
Appendix 7.4: Parameter estimates of select variables of GLMM on average outpatient expenditures

\begin{tabular}{|c|c|c|c|c|c|c|}
\hline \multirow{3}{*}{ Variables } & \multicolumn{3}{|c|}{ No Interaction } & \multicolumn{3}{|c|}{ With interaction } \\
\hline & \multicolumn{6}{|c|}{ Unadjusted analysis } \\
\hline & Beta & SE & Prob & Beta & SE & Prob \\
\hline Time & 0.40 & 0.04 & $<0.001$ & 0.41 & 0.04 & $<0.001$ \\
\hline ICI use & & & & N/A & & \\
\hline Yes & 1.66 & 0.05 & $<0.001$ & & & \\
\hline No & $(r e f)$ & & & & & \\
\hline Multimorbidity & & & & N/A & & \\
\hline Yes & 0.93 & 0.07 & $<0.001$ & & & \\
\hline No & $(r e f)$ & & & & & \\
\hline ICI-Multimorbidity Interaction & N/A & & & & & \\
\hline Yes ICI and Yes Multimorbidity & & & & 0.25 & 0.09 & 0.005 \\
\hline Yes ICI and No Multimorbidity & & & & $($ ref) & & \\
\hline No ICI and Yes Multimorbidity & & & & -1.26 & 0.08 & $<0.001$ \\
\hline No ICI and No Multimorbidity & & & & -2.24 & 0.11 & $<0.001$ \\
\hline \multirow[t]{4}{*}{ Time squared } & -0.07 & 0.01 & $<0.001$ & -0.07 & 0.01 & $<0.001$ \\
\hline & \multicolumn{3}{|c|}{ No Interaction } & \multicolumn{3}{|c|}{ With interaction } \\
\hline & \multicolumn{6}{|c|}{ Fully adjusted analysis ${ }^{\ddagger}$} \\
\hline & Beta & SE & Prob & Beta & SE & Prob \\
\hline Time & 0.17 & 0.03 & $<0.001$ & 0.18 & 0.03 & $<0.001$ \\
\hline ICI use & & & & N/A & & \\
\hline Yes & 1.18 & 0.07 & $<0.001$ & & & \\
\hline No & $(r e f)$ & & & & & \\
\hline Multimorbidity & & & & N/A & & \\
\hline Yes & 0.77 & 0.07 & $<0.001$ & & & \\
\hline No & $(r e f)$ & & & & & \\
\hline ICI-Multimorbidity Interaction & N/A & & & & & \\
\hline Yes ICI and Yes Multimorbidity & & & & 0.05 & 0.17 & 0.77 \\
\hline Yes ICI and No Multimorbidity & & & & $(r e f)$ & & \\
\hline No ICI and Yes Multimorbidity & & & & -0.97 & 0.16 & $<0.001$ \\
\hline No ICI and No Multimorbidity & & & & -1.79 & 0.17 & $<0.001$ \\
\hline Time squared & -0.06 & 0.01 & $<0.001$ & -0.06 & 0.01 & $<0.001$ \\
\hline
\end{tabular}

Note: Based on 6 repeated observations of 4,519 older adults with incident late-stage (Stage III/IV) melanoma continuously enrolled in Medicare Parts A and B fee-for-service programs 12 months prior to incident cancer diagnosis.

†Fully adjusted model also included year of diagnosis, age, sex, race, marital status, dual eligibility, oncologist visit, and primary care physician visits.

Probability $\mathrm{p}<0.05$ was considered significant

Abbreviations: GLMM, Generalized linear mixed model; ICI, Immune checkpoint inhibitors; N/A, Not applicable; Prob, Probability; ref, Reference; SE, Standard error 
Appendix 7.5: Parameter estimates of select variables of GLMM on average home healthcare expenditures

\begin{tabular}{|c|c|c|c|c|c|c|}
\hline \multirow{3}{*}{ Variables } & \multicolumn{3}{|c|}{ No Interaction } & \multicolumn{3}{|c|}{ With interaction } \\
\hline & \multicolumn{6}{|c|}{ Unadjusted analysis } \\
\hline & Beta & SE & Prob & Beta & SE & Prob \\
\hline Time & 0.33 & 0.06 & $<0.001$ & 0.33 & 0.06 & $<0.001$ \\
\hline ICI use & & & & N/A & & \\
\hline Yes & 0.23 & 0.13 & 0.820 & & & \\
\hline No & $(r e f)$ & & & & & \\
\hline Multimorbidity & & & & N/A & & \\
\hline Yes & 1.64 & 0.16 & $<0.001$ & & & \\
\hline No & $(r e f)$ & & & & & \\
\hline ICI-Multimorbidity Interaction & N/A & & & & & \\
\hline Yes ICI and Yes Multimorbidity & & & & 0.81 & 0.31 & 0.009 \\
\hline Yes ICI and No Multimorbidity & & & & $(r e f)$ & & \\
\hline No ICI and Yes Multimorbidity & & & & 0.79 & 0.30 & 0.008 \\
\hline No ICI and No Multimorbidity & & & & -0.93 & 0.34 & 0.006 \\
\hline \multirow[t]{3}{*}{ Time squared } & -0.05 & 0.01 & $<0.001$ & -0.06 & 0.01 & $<0.001$ \\
\hline & \multicolumn{6}{|c|}{ Fully adjusted analysis ${ }^{\ddagger}$} \\
\hline & Beta & SE & Prob & Beta & SE & Prob \\
\hline Time & 0.19 & 0.06 & 0.002 & 0.20 & 0.07 & 0.002 \\
\hline ICI use & & & & N/A & & \\
\hline Yes & 0.12 & 0.25 & 0.622 & & & \\
\hline No & $(r e f)$ & & & & & \\
\hline Multimorbidity & & & & N/A & & \\
\hline Yes & 1.21 & 0.19 & $<0.001$ & & & \\
\hline No & $(r e f)$ & & & & & \\
\hline ICI-Multimorbidity Interaction & N/A & & & & & \\
\hline Yes ICI and Yes Multimorbidity & & & & 0.06 & 0.62 & 0.928 \\
\hline Yes ICI and No Multimorbidity & & & & $(r e f)$ & & \\
\hline No ICI and Yes Multimorbidity & & & & 0.24 & 0.61 & 0.696 \\
\hline No ICI and No Multimorbidity & & & & -1.07 & 0.63 & 0.092 \\
\hline Time squared & -0.05 & 0.01 & $<0.001$ & -0.05 & 0.01 & $<0.001$ \\
\hline
\end{tabular}

Note: Based on 6 repeated observations of 4,519 older adults with incident late-stage (Stage III/IV) melanoma continuously enrolled in Medicare Parts A and B fee-for-service programs 12 months prior to incident cancer diagnosis.

†Fully adjusted model also included year of diagnosis, age, sex, race, marital status, dual eligibility, oncologist visit, and primary care physician visits.

Probability $\mathrm{p}<0.05$ was considered significant

Abbreviations: GLMM, Generalized linear mixed model; ICI, Immune checkpoint inhibitors; N/A, Not applicable; Prob, Probability; ref, Reference; SE, Standard error 
Appendix 7.6: Parameter estimates of select variables of GLMM on average inpatient expenditures

\begin{tabular}{|c|c|c|c|c|c|c|}
\hline \multirow{3}{*}{ Variables } & \multicolumn{3}{|c|}{ No Interaction } & \multicolumn{3}{|c|}{ With interaction } \\
\hline & \multicolumn{6}{|c|}{ Unadjusted analysis } \\
\hline & Beta & SE & Prob & Beta & SE & Prob \\
\hline Time & 1.94 & 0.17 & $<0.001$ & 1.93 & 0.17 & $<0.001$ \\
\hline ICI use & & & & N/A & & \\
\hline Yes & 0.68 & 0.17 & $<0.001$ & & & \\
\hline No & $($ ref) & & & & & \\
\hline Multimorbidity & & & & N/A & & \\
\hline Yes & 1.05 & 0.15 & $<0.001$ & & & \\
\hline No & $(r e f)$ & & & & & \\
\hline ICI-Multimorbidity Interaction & N/A & & & & & \\
\hline Yes ICI and Yes Multimorbidity & & & & 0.20 & 0.34 & 0.551 \\
\hline Yes ICI and No Multimorbidity & & & & $(r e f)$ & & \\
\hline No ICI and Yes Multimorbidity & & & & -0.27 & 0.29 & 0.342 \\
\hline No ICI and No Multimorbidity & & & & -1.40 & 0.32 & $<0.001$ \\
\hline \multirow[t]{4}{*}{ Time squared } & -0.34 & 0.03 & $<0.001$ & -0.33 & 0.03 & $<0.001$ \\
\hline & \multicolumn{3}{|c|}{ No Interaction } & \multicolumn{3}{|c|}{ With interaction } \\
\hline & \multicolumn{6}{|c|}{ Fully adjusted analysis ${ }^{\ddagger}$} \\
\hline & Beta & SE & Prob & Beta & SE & Prob \\
\hline Time & 1.56 & 0.22 & $<0.001$ & 1.56 & 0.22 & $<0.001$ \\
\hline ICI use & & & & N/A & & \\
\hline Yes & -0.07 & 0.16 & 0.676 & & & \\
\hline No & (ref) & & & & & \\
\hline Multimorbidity & & & & N/A & & \\
\hline Yes & 0.63 & 0.25 & 0.011 & & & \\
\hline No & $($ ref) & & & & & \\
\hline ICI-Multimorbidity Interaction & N/A & & & & & \\
\hline Yes ICI and Yes Multimorbidity & & & & 0.02 & 0.31 & 0.961 \\
\hline Yes ICI and No Multimorbidity & & & & $($ ref) & & \\
\hline No ICI and Yes Multimorbidity & & & & 0.21 & 0.29 & 0.463 \\
\hline No ICI and No Multimorbidity & & & & -0.46 & 0.35 & 0.179 \\
\hline Time squared & -0.30 & 0.04 & $<0.001$ & -0.30 & 0.04 & $<0.001$ \\
\hline
\end{tabular}

Note: Based on 6 repeated observation of 4,519 older adults with incident late-stage (Stage III/IV) melanoma continuously enrolled in Medicare Parts A and B fee-for-service programs 12 months prior to incident cancer diagnosis.

ҒFully adjusted model also included year of diagnosis, age, sex, race, marital status, dual eligibility, oncologist visit, and primary care physician visits.

Probability $\mathrm{p}<0.05$ was considered significant

Abbreviations: GLMM, Generalized linear mixed model; ICI, Immune checkpoint inhibitors; N/A, Not applicable; Prob, Probability; ref, Reference; SE, Standard error 